\title{
Selected Abstracts from the PICS Society Symposium 2021 Aria Convention Center Las Vegas, NV September 1-4, 2021
}

\begin{tabular}{l} 
Directors \\
\hline Dr. Ziyad M. Hijazi \\
Dr. John P. Cheatham \\
Dr. Thomas Jones \\
Dr. Damien Kenny \\
Dr. Carlos Pedra \\
Dr. Evan Zahn
\end{tabular}

Co-Directors

Dr. Felix Berger

Dr. Allison Cabalka

Dr. John D. Carroll

Dr. Matt Gillespie

Dr. Ralf Holzer

Dr. Clifford Kavinsky

Dr. Daniel Levi

All content was reviewed and selected by the Review Committee, which held full responsibility for the abstract selections.

\section{Valve-in-Valve Transcatheter Aortic Valve} Replacement for a Failed Bioprosthetic Valve in a Patient with Repaired Truncus Arteriosus and Right-Sided Aortic Arch

$\underline{\text { Lina Ya'qoub }}^{1,2}$, Tarek Helmy ${ }^{2}$, Rodney Reeves ${ }^{2}$, Steven Bailey ${ }^{2}$

${ }^{1}$ Henry Ford, Detroit, USA. ${ }^{2}$ Ochsner-Louisiana State University, Shreveport, USA

We are seeing more patients with repaired congenital cardiac diseases. These patients are often complex requiring careful clinical evaluation. In this case report, we present a very interesting case in a patient with repaired truncus arteriosus and right-sided aortic arch, who was hospitalized at our institution for recurrent heart failure exacerbation. He was found to have severe eccentric aortic valve regurgitation due to failed bioprosthetic valve. Given his multiple cardiac surgeries, he was deemed high risk for surgery and underwent successful valve-in-valve transcatheter aortic valve replacement with immediate improvement in his hemodynamics and symptoms.

\section{ETHICS DECLARATIONS}

Conflict of interest: The authors declare that they do not have any conflict of interests.

Ethical Approval: Case abstract is presented within the ethical guidelines. There is no identifying health information.

Consent for Publication: Not applicable. 


\section{Transcatheter Mitral Repair in Children: a MitraClip Case Series}

\author{
$\underline{B r e n a ~ H a u g h e y}^{1}$, Dale Fowler ${ }^{2}$, Michael Hainstock ${ }^{1}$, Scott Lim ${ }^{1,2}$ \\ ${ }^{1}$ University of Virginia, Department of Pediatrics (Cardiology), \\ Charlottesville, USA. ${ }^{2}$ University of Virginia, Department \\ of Medicine (Cardiovascular Medicine), Charlottesville, USA
}

For pediatric patients with severe mitral regurgitation (MR) despite medical therapy and at prohibitive surgical risk, there are few alternatives. In adult patients with severe MR, transcatheter mitral repair (TMVr) with MitraClip ${ }^{\mathrm{TM}}$ is approved for patients with primary/degenerative disease at high surgical risk, and for all patients with secondary/functional MR failing medical therapy. Out of $>1000$ cases of TMVr by our multidisciplinary valve team, we treated three pediatric patients $(6,17$, and 17 years) from 2013 to 2020 with severe MR failing medical therapy who after review by the multidisciplinary team were felt to be at prohibitive surgical risk. Two patients were with degenerative mitral regurgitation, and one patient had functional MR despite maximally tolerated heart failure medications. Two patients transferred from another institution on inotropic support. All patients underwent their $\mathrm{TMVr}$ procedure from a transvenous/transseptal approach, with transesophageal echocardiographic (TEE) guidance, implanting an average of 1.3 clips/case (utilizing classic MitraClip ${ }^{\mathrm{TM}}$ NT in one, Generation 3 XTR in one, and Generation 4 NTW in the third). Inotropic support was weaned off in 2.5 days on average in the patients requiring pre-intervention support. All patients were then discharged to home without complications from the procedure after an average hospitalization of 5 days. At an average follow up of 38 months (range 6-96 months), all patients continue to do well without symptoms (NYHA I) on reduced, or similar oral heart failure medication. Left ventricular ejection fraction demonstrated an average 11\% improvement at follow up. Maximum residual MR at follow up was mild to moderate. No complications from the procedure were noted. TMVr with MitraClip ${ }^{\mathrm{TM}}$ was safely performed and efficacious in this early case series of pediatric patients at prohibitive surgical risk.
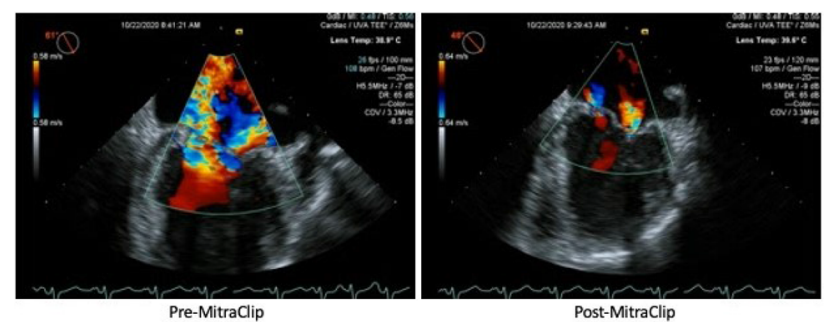

ETHICS DECLARATIONS

Conflict of interest: The authors declare that they do not have any conflict of interests.

Ethical Approval: Not applicable.

Consent for Publication: Not applicable.

\section{My Nightmare Case in Cath Lab-Retrieval of Embolized ASD from LA Using Innovative Techniques}

\section{Amjad Mahmood}

AFIC, Rawalpindi, Pakistan

This case report is aimed to present various innovative techniques employed to retrieve the embolized ASD device from LA. The procedures involved were very unconventional, safe and very promising using multiple snares, catheters and sheaths and artery forceps. Embolization of the devices may be due to several reasons varying from improper estimation (size, dimension, alignment and axis) to problems with image quality just before device release. Once embolized, the retrieval depends upon the type, site, size and position of the device. Moreover, availability of the appropriate sheaths, variety of snares, bioptomes, balloons, biplane fluoroscopy and lot of patience is the prerequisite for device retrieval. In this case we used $34 \mathrm{~mm}$ cocoon ASD device to close ASD which was thoroughly evaluated by TTE and TEE prior to procedure and found to have suitable rims. The device embolized few hours after implantation in to LA probably due to malaligned atrial septum. During device retrieval, incidentally device was captured at a stage when the snare loaded catheter was not within the sheath. We tried our best to take the snare with captured device into the sheath in repeated attempts but there was chance of losing the device. Attempts to retrieve from another sheath with the help of snare could not succeed due to acute angle between sheath and snare catheter. We tried to milk the device into the IVC without sheath by gently angulating and pulling. While at the mouth of IVS with the help of different snares (Maslanka, Gooseneck, Ensnare, Delivery cable of Occlutech device, Endomyocardial biopsy catheter) we successful by pulled the device up to groin. At this point due to nonavailability of appropriate size $14 \mathrm{~F}$ small sheath, we took the device in the femoral vein just below skin with the help of snare. Venesection done just above device and proximal knob was held with the help of artery forceps which was clamped. Before pulling the device, stay silk sutures of figure of eight were applied one below and two above the device. While gently pulling the device out of skin figure of eight sutures were tightened and the field was bloodless following device retrieval. Post procedure the patient remained stable and referred to cardiac surgeon for patch closure of the ASD. The device was successfully retrieved using different snares, catheters, artery forceps and hemostatic techniques to avoid blood loss. Peri-procedure the patient remained stable hemodynamically, there was no arrhythmia and desaturation. Post procedure course remained uneventful too. Patient was discharged the next day and referred to cardiac surgeon for ASD patch closure. Device embolization can be prevented with accurate preprocedure assessment and thorough estimation of position, stability, and residual flow judgment on color with good imaging during deployment. Device retrieval is safe if all the required equipment and expertise is available. 
ETHICS DECLARATIONS

Conflict of interest: The authors declare that they do not have any conflict of interests.

Ethical Approval: Approved from IERB.

Consent for Publication: Not applicable.

\section{New Expandable Polyurethane Stent Valve, Implanted by Catheter, in Pediatric and Adult Patients}

\author{
Miguel Maluf \\ São Paulo Federal University, São Paulo, Brazil
}

Patients with pediatric prostheses suffer from mismatch and early calcification, which causes a high number of reoperations. Expandable Polyurethane Stent Valve (EPSV), is composed by a flexible polyurethane (PU) leaflets is grown on the top of an expandable cobalt-chrome alloy stent, including the formation of three leaflets. Physical, Hydrodynamic, Animal studies, were performed following: ISO 5840-3, 2015. Physical tests. Result of study of surface scanning of pre and post crimp stent, showed no structural modification of the PU. Hydrodynamic test showed a pressure gradient oscillation between 5 and $20 \mathrm{~mm}$, in basal or stress condition, respectively. Experimental studies. Sheep were subjected to 3D echo-Doppler study, in 6th follow-up months, which showed satisfactory hemodynamic performance, with low transvalvular gradient $(\mathrm{M}=6.60 \mathrm{mmHg})$. Six stents were explanted after 20 days to 21 months of follow-up to Ultrastructural analysis. All of which revealed no presence of calcium growth and prosthesis structure was intact. Association between stent-design and PU-leaflets, allowed acceptable hemodynamic performance, regardless of the stent valve diameter. The resistance of polyurethane to crimping and the lack of calcification of leaflets, are advantages that will allow greater durability of the prosthesis. This new concept of synthetic expandable stent valve could be a viable option in growing patients.

ETHICS DECLARATIONS

Conflict of interest: The authors declare that they do not have any conflict of interests.

Ethical Approval: Not applicable.

Consent for Publication: Not applicable.

\section{Bioptome Assisted Stent Repositioning in a Case of Proximal Stent Migration During Balloon Expandable Stenting for Coarctation of the Aorta}

\section{Neeraj Awasthy}

Max Hospital, Delhi, India

Endovascular stenting of aortic coarctation is now an accepted technique to relive obstruction. Only a few bail out techniques such as stent deployment at distal sites or balloon assisted techniques for stent redeployment have been described. A 60 year old lady present with hypertension and on evaluation was found to have systemic hypertension. She underwent computerized tomography (CT) for evaluation. This was followed by stenting and proximal stent migration. The stent was successfully redeployed using a novel Bioptome assisted coarctation stent reposition. We also propose the concept of coarctation mal-alignment between the proximal and distal parts of stent as the underlying precipitant cause.

ETHICS DECLARATIONS

Conflict of interest: The authors declare that they do not have any conflict of interests.

Ethical Approval: Not applicable.

Consent for Publication: Not applicable.

\section{Incidence and Risk Factors for Hematochezia in Shunt-Dependent Single Ventricle Patients After Cardiac Catheterization}

\section{Veronica Zitterman, Emily Miller, Jeffrey Zampi, Sunkyung Yu} Michigan Medicine, Ann Arbor, USA

Infants with single ventricle congenital heart disease, especially those with shunt physiology, are at risk for gastrointestinal complications including feeding intolerance, failure to thrive, and necrotizing enterocolitis. This may be secondary to poor gut perfusion due to a high Qp:Qs, low aortic diastolic pressures due to shunt run-off, and/or residual aortic arch obstruction. We observed several patients who developed hematochezia following cardiac catheterization procedures prior to stage II surgical palliation (partial/superior cavopulmonary anastomosis). Therefore we sought to identify any patient or procedural risk factors for post-catheterization hematochezia in the shuntdependent single ventricle population. A retrospective case-control study was performed on all shunt-dependent single ventricle patients who developed hematochezia after pre-stage II cardiac catheterization at our center from 2014 to 2018 . Cases were matched 1:4 with controls of shunt-dependent single ventricle patients who underwent prestage II cardiac catheterization without hematochezia by diagnosis, type of initial palliative procedure, and age at catheterization. Anatomic, physiologic, and catheterization technical elements were examined for all patients. Standard descriptive statistics were used to compare cases and controls. Of a total of 60 patients with pre-stage II cardiac catheterization reviewed, post-catheterization hematochezia was observed in 7 patients, with 5 meeting all inclusion criteria. Thus the overall incidence was $8.3 \%$ in shunt-dependent single-ventricle patients over a 5 year time frame. Of these 5 , the stage II cardiac surgery was delayed as a result in 4 patients and 2 patients were diagnosed with necrotizing enterocolitis stage $1 \mathrm{~B}$ or $2 \mathrm{~A}$. Compared to the 20 controls, less restrictive pulmonary blood flow was the primary physiologic determinant of hematochezia, as no patients with hematochezia had echocardiographic or angiographic shunt narrowing and none required post-procedure supplemental oxygen, compared to $28 \%, 35 \%$ and $60 \%$, respectively, in the control patients. No hemodynamic or anatomic variables appeared to be associated with hematochezia with similar measured Qp:Qs, arteriovenous oxygen saturation difference, and systemic ventricular end diastolic pressure between groups. Similarly, there were no apparent catheterization related risks for hematochezia with similar systemic heparinization doses, activated clotting times, and anesthetic management across groups. Examining non-cardiac and non-technical determinants of hematochezia, although a prior history of hematochezia was not more common in the hematochezia group, more patients with hematochezia were on high caloric concentration feeds, with $80 \%$ of patients on $26 \mathrm{kcal} / \mathrm{oz}$ or higher feeds and $40 \%$ on over $28 \mathrm{kcal} / \mathrm{oz}$ feeds (in comparison to $45 \%$ and $10 \%$ in the control group). Although 
hematochezia is a rare post-catheterization complication, it can have an important impact on shunt-dependent single ventricle patients, can present as necrotizing enterocolitis, and can alter the timing of cardiac surgery. Potential risk factors for post cardiac catheterization hematochezia are less restrictive shunt physiology and high caloric density feeds (greater than $24 \mathrm{kcal} / \mathrm{oz}$ ). However, as no other anatomic, physiologic, or technical associations were found, prevention may not be feasible.

\section{ETHICS DECLARATIONS}

Conflict of interest: The authors declare that they do not have any conflict of interests.

Ethical Approval: Not applicable.

Consent for Publication: Not applicable.

\section{Device Closure of Large Perimembranous Ventricular Septal Defects $(\geq 6 \mathrm{~mm})$ Using the LEPU MEMOPART SYMMETRIC MEMBRANOUS VSD OCCLUDER: Short and Midterm Results}

\author{
Rajesh Kumar Ramaswamy, Ramyashri Chandrasekhar, \\ Vivekan Manoharan
}

\section{MGM Health Care, Chennai, India}

The aim of this study is to evaluate the safety and efficacy of transcatheter device closure of moderate to large perimembranous ventricular septal defects $\geq 6 \mathrm{~mm}$ using LEPU MEMOPART SYMMETRIC MEMBRANOUS VSD OCCLUDER in pediatric patients at short and mid-term follow-up. We prospectively studied 50 patients with large perimembranous VSDs ( $\geq 6 \mathrm{~mm}$ ) between August 2019 and May 2021 who underwent percutaneous closure at our centre. Transthoracic echocardiography (TTE) and electrocardiogram were done before and after the procedure. All patients were subjected to follow-up evaluation at $48 \mathrm{~h}, 1,3,6,12$ months and annually thereafter with TTE and electrocardiogram. A total of 50 patients (30 males and 20 females) underwent transcatheter closure of large perimembranous VSD $\geq 6 \mathrm{~mm}$. Mean age of patients was 26 months (range 5-180 months) and mean weight was 13.4 (range 5.3-28 kg). $25 \%$ had large PM VSD with inlet extension. The mean defect diameter on color flow mapping on TTE was $9.4(7-14 \mathrm{~mm})$ the pulmonary to systemic blood flow (Qp/Qs) was 32.1 (range 2.0 to 2.6). The device diameter ranged from 7 to $16 \mathrm{~mm}$ (median $=9 \mathrm{~mm}$ ). The procedure was carried out successfully in $96 \%$ of patients with no reported mortality. Two patients with associated mild aortic valve prolapse developed mild aortic regurgitation so device could not be successfully deployed and referred for surgery. During the catheterization, there were only minor complications and at follow-up of $10 \pm 5.1$ (1-22 months), the closure rate was high of $96 \%$ and freedom from AV block was $100 \%$. A minimal residual shunt seen as a thin streak on transthoracic color flow mapping persisted in $2(4 \%)$ patients, which remained unchanged over a follow-up period. $5(10 \%)$ patients had trivial AR and $8(16 \%)$ patients had mild TR pre-procedure which did not worsen on follow up. Two patients developed moderate TR which was mild TR pre-procedure not worsen on follow up. There were no other device related complications such as device migration, systemic thromboembolism, infective endocarditis, pericardial effusion or delayed conduction disturbances. The LEPU MEMOPART SYMMETRIC MEMBRANOUS VSD OCCLUDER offers excellent closure rates and low morbidity when used to close large perimembranous VSDs. The device appears to be safe and effective in short and mid-term follow-up. Long term follow up is necessary to establish the effectiveness of these type of device in these particular subset of VSDs.

ETHICS DECLARATIONS

Conflict of interest: The authors declare that they do not have any conflict of interests.

Ethical Approval: Not applicable.

Consent for Publication: Not applicable.

\section{Triplett Melody in Tune-Recruiting a Rudimentary Native Right Ventricular Outflow Tract for Implanting a Melody Valve in Addition to a Repeat Melody Valve-in-Valve Implantation into a Failing Hancock Conduit in a Lady After Tetralogy of Fallot Repair}

Heike Schneider, Matthias Mueller, Claudia Dellas, Thomas Paul University Medicine Goettingen, Goettingen, Germany

We present a 53-year-old lady with Tetralogy of Fallot. At the age of 10 years, a left-sided modified Blalock-Taussig-Thomas shunt had been performed at another institution. 20 years later (1998), she had undergone corrective surgery with implantation of a $20 \mathrm{~mm}$ Han$\operatorname{cock}{ }^{\circledR}$ conduit and reconstruction of the left pulmonary artery. For atypical anatomy, the conduit had been placed anteriorly as the native right ventricular outflow tract had not been reached by the surgeon.Eleven years ago, she had presented with frank right heart failure and ventricular tachycardia due to a severely stenotic and regurgitant conduit. A Melody ${ }^{\circledR}$ valve (no. 1) was implanted but a residual gradient of $18 \mathrm{mmHg}$ was evident due to the narrow Hancock conduit with the valve being competent. An endocardial ICD system was implanted subsequently. Her clinical status improved quickly. Despite several invitations, she did not show up to follow-up visits until recently, when she again presented with frank right heart failure, dyspnea and massive ascites. Cardiac catheterization was performed and revealed elevated right heart filling pressure of $27 \mathrm{mmHg}$. RV pressure was supra-systemic with a gradient from RV to PA artery of $50 \mathrm{mmHg}$ in face of poor RV function. In addition significant Melody insufficiency and LPA stenosis were evident. LPA stenosis was handled by stent implantation. For conduit failure a prestent covering the RVOT was implanted followed by a second Melody ${ }^{\circledR}$ (no. 2) resulting in gradient reduction to only $30 \mathrm{mmHg}$. Repeat PA angiography with a competent Melody valve nicely delineated anatomy of the tiny native RVOT measuring $8 \mathrm{~mm}$ at its narrowest diameter. A covered and an uncovered $\mathrm{CP}$ stent were implanted into the RVOT establishing a landing zone of $18 \mathrm{~mm}$. A third Melody was subsequently implanted eliminating RV-to-PA gradient. Both valves were competent. The patient recovered slowly.

ETHICS DECLARATIONS

Conflict of interest: The authors declare that they do not have any conflict of interests.

Ethical Approval: Not applicable.

Consent for Publication: Not applicable. 


\section{Echo-Guided Balloon Valvuloplasty of the Aortic Valve in Neonates and Infants Reduces Contrast Exposure While Maintaining Safety and Efficacy}

\author{
Conor O'Halloran, Sandhya Ramlogan, Nazia Husain, Alan \\ Nugent, Paul Tannous
}

\section{Ann \& Robert H. Lurie Children's Hospital of Chicago, Chicago, USA}

Balloon aortic valvuloplasty (BAV) is the preferred treatment in neonates and infants with clinically significant aortic stenosis (AS). Traditionally, contrast angiography is used to measure the annulus and to assess for aortic regurgitation (AR) after each balloon inflation. Intraprocedural echocardiography now guides other pediatric interventions, and our group has recently adopted this strategy for BAV. It is hypothesized this will reduce exposure to iodinated contrast and ionizing radiation, without compromising efficacy and complications. All patients $<10 \mathrm{~kg}$ undergoing BAV at a single institution from 2013 to 2020 were retrospectively investigated. Demographic, clinical, echocardiographic, and procedural details were extracted from the medical record. Agreement between echocardiographic and angiographic aortic valve annulus measurements was assessed using a twoway intraclass correlation coefficient. Technical success was defined as final invasive gradient $<35 \mathrm{mmHg}$ and mild or less AR by echocardiography. Outcomes were compared between echocardiogram guided BAV (eBAV) and traditional BAV (tBAV) using linear regression to control for patient weight, presence of critical AS, and presence of other congenital heart disease. 6 patients received eBAV and 19 patients received $\mathrm{tBAV}$ at our institution during the study period. Median age was 39 days (IQR 20-72), median weight was $4.4 \mathrm{~kg}$ (IQR 3.1-5.1), 4 patients $(16 \%)$ had critical AS and 8 patients $(32 \%)$ had other congenital heart disease. The mean annulus size was $7.5 \mathrm{~mm}$ by angiography and the mean invasive gradient was $54 \mathrm{mmHg}$. There were no significant differences between eBAV and tBAV groups with regards to these baseline characteristics. There was very strong correlation between annulus measurements by intraprocedural echocardiogram and angiography (ICC $0.96, p=0.002$ ) in contrast to preprocedural echocardiogram and angiography (ICC $0.73, \mathrm{p}<0.001$ ). eBAV patients received significantly less contrast $(0.6 \mathrm{vs} 3.5 \mathrm{ml} / \mathrm{kg}, \mathrm{p}=0.002)$. One eBAV patient $(17 \%)$ experienced a serious adverse event (blood transfusion), while serious adverse events occurred in 3/19 tBAV patients $(16 \%, p=0.97)$. Technical success was achieved in $6 / 6$ eBAV patients $(100 \%)$ and $16 / 19$ tBAV patients $(84 \%, p=0.29)$. There were no differences in final invasive gradient, mean gradient by follow up echocardiogram, or change in fractional shortening after BAV. None of the eBAV patients developed increased AR after BAV compared to $8 / 18$ tBAV patients (44\%) with available data, though this was not statistically significant $(\mathrm{p}=0.08)$. There was no difference in sheath time, number of balloons used, or maximum balloon to annulus ratio. Despite echocardiographic guidance being associated with longer fluoroscopic time ( $29 \mathrm{vs} 17 \mathrm{~min}, \mathrm{p}=0.035$ ) the total radiation dose area product was similar between the eBAV and tBAV groups (180 vs $\left.459 \mathrm{mGy} \cdot \mathrm{cm}^{2}, \mathrm{p}=0.19\right)$. eBAV is as safe and effective as tBAV and was associated with lower contrast exposure. In our early experience the total radiation dose was similar even though there were longer fluoroscopy times, likely due to reduced cineangiography. There was strong agreement between aortic valve annulus measurements by intraprocedural echocardiogram and angiography. It is likely that real time, nonionizing imaging will replace angiography for annular measurement, and assessment of post dilation AR thus allowing for contrast-free BAV.

\section{ETHICS DECLARATIONS}

Conflict of interest: The authors declare that they do not have any conflict of interests.

Ethical Approval: The IRB at Ann \& Robert H. Lurie Children's Hospital approved this study, with waiver of the need for informed consent.

Consent for Publication: Not applicable.

\section{Inferior-Superior Transcatheter Valve Placement in a Branch Pulmonary Artery}

\author{
Joshua Kurtz $^{1,2}$, Melissa Perrotta ${ }^{1,2}$, Walter Sobczyk ${ }^{1,2}$, Edward \\ $\operatorname{Kim}^{1,2}$ \\ ${ }^{1}$ University of Louisville, Louisville, USA. ${ }^{2}$ Norton Children's \\ Hospital, Louisville, USA
}

Transcatheter pulmonary valve replacement (TPVR) is increasingly used to treat complications related to dysfunctional right ventricular outflow tracts (RVOT). Candidacy with currently available TPVR is limited by RVOT diameter and coronary artery anatomy. Placing a TPVR in each of the branch pulmonary arteries (BPA) is emerging as a viable strategy for those ineligible for traditional TPVR. We present the case of a BPA that was too large to accommodate a single TPVR and underwent placement of 2 TPVR in a single BPA in a superiorinferior orientation and a single TPVR in the other BPA. The patient is a 54-year-old man with a history of pulmonary valve stenosis who underwent a Brock procedure at 8 years-old. He has recurrent atrial flutter and fibrillation that has required multiple ablations, obstructive sleep apnea, hypertension, and hypercholesterolemia. He had been lost to follow up, but represented for medical care recently due to NYHA class 3 heart failure symptoms. He underwent a cardiac MRI which showed an RVEDVi of $120 \mathrm{ml} / \mathrm{m}^{2}$, LVEDVi of $79 \mathrm{ml} / \mathrm{m}^{2}$, RVEF of $50 \%$, LVEF of $57 \%$, and pulmonary regurgitant fraction of $36 \%$. Peak instantaneous gradient across the pulmonary valve was $41 \mathrm{mmHg}$ by echo. A metabolic stress test showed a peak VO2 of $11.4 \mathrm{ml} / \mathrm{kg} / \mathrm{min}$. He was recommended to undergo TPVR because he was a poor surgical candidate due to comorbidities. Coronary compression testing showed distortion of the right coronary cusp preventing traditional TPVR. He had a dilated, dynamic right pulmonary artery (RPA) that measured $36 \mathrm{~mm}$. Balloon sizing of the RPA using 2, $18 \mathrm{~mm}$ Z-Med II balloons was performed. Based on this 2 Cook XL 4010 stents were deployed simultaneously in a superiorinferior manner in the RPA, one deployed at $18 \mathrm{~mm}$ and the other deployed at $20 \mathrm{~mm}$. The inferior stent began to migrate distally and wire position was lost when trying to position a second stent interlocking. Right internal jugular access as obtained and wire position re-established through the inferior stent. A Cook XL 5010 stent was then deployed to secure the inferior stent. A $22 \mathrm{~mm}$ Melody valve was deployed in the inferior stent on a $20 \mathrm{~mm}$ Ensemble delivery system and a $22 \mathrm{~mm}$ Melody valve was deployed in the superior stent mounted on an $18 \mathrm{~mm}$ Ensemble delivery. He returned to the cath lab 5 weeks later. At that time an angiogram showed good function of the valves with trivial insufficiency. Balloon sizing was performed with a $26 \mathrm{~mm} \times 3 \mathrm{~cm}$ ZMedII balloon in the left pulmonary artery (LPA). A Palmaz XL 5010 stent was deployed in the LPA at $30 \mathrm{~mm}$. A $26 \mathrm{~mm}$ Edwards Sapien 3 valve was then deployed into the stent on a $26 \mathrm{~mm}$ Commander delivery system. This case demonstrates that a "double valve" strategy is feasible when the native vessel cannot accommodate current TPVR. Satisfactory valve function is present at 
early follow up. This case emphasizes the importance of maintaining stent stability and wire position at all times.

\section{ETHICS DECLARATIONS}

Conflict of interest: The authors declare that they do not have any conflict of interests.

Ethical Approval: Not applicable.

Consent for Publication: Not applicable.

\section{The BeGraft Aortic Covered Stent for Coarctation of the Aorta: Acute and Intermediate Follow-Up Results}

\section{Elchanan Bruckheimer, Tamir Dagan, Gabriel Amir, Alex Loewenthal, Einat Birk}

Schneider Children's Medical Center Israel, Petach Tikva, Israel

Report experience with the new premounted BeGraft Aortic covered stent (Bentley; Hechingen, Germany) for the treatment of coarctation of the aorta. Transcatheter treatment of coarctation of the aorta with covered stents is safe and effective and reduces the probability of aortic wall injury. However the need for relatively large delivery systems and risk for femoral arterial damage, rigid stents and flimsy PTFE covering are significant limitations for use and success. The BeGraft Aortic Stent Graft System consists of a $0.035^{\prime \prime}$ guide wirecompatible stent delivery catheter with a premounted ePTFE-covered open-cell balloon-expandable Cobalt Chromium stent. Balloon sizes range from $12[9 \mathrm{Fr}$ ] to $24 \mathrm{~mm}[14 \mathrm{Fr}$ ] and stent length ranges from 19 to $59 \mathrm{~mm} .60$ patients [ $42 \mathrm{M}, 18 \mathrm{~F}$ ] of median age 14.7 years [4.1-52.8] and weight $52.3 \mathrm{~kg}$ [14.5-104.7] with coarctation of the aorta, 26 native, 34 with previous interventions [22 s/p surgical repair, $15 \mathrm{~s} / \mathrm{p}$ bare stent, $9 \mathrm{~s} / \mathrm{p}$ covered stent] and 8 aneurysms, underwent transcatheter stent implantation via a $9-11 \mathrm{Fr}$ femoral arterial access. BeGraft Aortic Stents used included $12 \mathrm{~mm}$ [48] $14 \mathrm{~mm} \mathrm{[7]} \mathrm{and} 16 \mathrm{~mm} \mathrm{[5]} \mathrm{and} \mathrm{these} \mathrm{were} \mathrm{post} \mathrm{dilated} \mathrm{up} \mathrm{to} 18 \mathrm{~mm}$ through the same delivery sheath as needed. All stents [65 in total] were successfully implanted and post-dilated in all cases. Systolic peak pressure gradient decreased from $20.3 \pm 8.8$ to $4.2 \pm 4.3 \mathrm{mmHg}[\mathrm{p}<0.001]$ and the diameter of the coarctation increased from $7.8 \pm 3.6$ to $13.6 \pm 2.2 \mathrm{~mm}[\mathrm{p}<0.001]$. The final stent: transverse arch diameter was $1.0 \pm 0.1$. There was no acute aortic wall injury nor other immediate complications and all 8 patients underwent successful occlusion of the pre-existing aneurysm. At median follow-up of 21.1 months [0.1-50.4] all patients are alive. 3 patients underwent scheduled further dilation in the first year post-implantation. 1 patient had endovasculitis which resolved with antibiotics but developed an aneurysm which was treated with a second Begraft stent. 11 patients have hypertension. These results of treatment of types of coarctation of the aorta with the BeGraft Aortic Stent are encouraging. The advantages of a relatively low profile [9$11 \mathrm{Fr}]$ delivery system for a stent that can be post-dilated allows for safer use in smaller patients. The PTFE covering was successful in treating aneurysms and the lack of significant recoil is indicated by the final stent diameter relative to the transverse arch. Long term outcome of blood pressure control, stent integrity and prevention of aortic wall injury need to be evaluated.

ETHICS DECLARATIONS

Conflict of interest: Elchanan Bruckheimer is a consultant for Bentley.

Ethical Approval: Not applicable.

Consent for Publication: Not applicable.
012. Transcatheter Mitral Valve-in-Valve Implantation in Pediatric Patients

\author{
Mohamed Al Nassef $^{1}$, Atif Al Sahari ${ }^{2}$, Saleem Ahmad ${ }^{3}$, Tarek \\ Momenah $^{1,4}$ \\ ${ }^{1}$ Prince Sultan Cardiac Center, Riyadh, Saudi Arabia. ${ }^{2}$ Prince sultan \\ Cardiac Center, Riyadh, Saudi Arabia. ${ }^{3}$ Prince Sultan Cardiac Center, \\ Riyadh, Saudi Arabia. ${ }^{4}$ Al Faisal University, Riyadh, Saudi Arabia
}

Transcatheter implantation of Edward's valve within the bioprosthetic mitral valve is an established method of treatment in adults. However, it is not well studied in the pediatric age group. We reviewed our experience with mitral valve implantation in this cohort. Transcatheter mitral valve implantation was attempted in four symptomatic patients with dysfunctional mitral valve bioprosthesis implanted at an earlier stage due to severe mitral valve stenosis or regurgitation. The mean age and weight of the patients at the time of the procedure were 11.4 years (range 10-14) and $36 \mathrm{~kg}$ (range 31-44), respectively The transmitral mean gradient dropped from a mean of $19.75 \mathrm{mmHg}$ (range 15-22) to a mean of $1 \mathrm{mmHg}$ (range 0-3) after the procedure. The mean fluoroscopy time was 55.25 min (range 40-72), and the mean hospital length of stay was 4 days (Range 3-7). The patients' functional class improved from NYHA class IV to I during the follow-up period. Transcatheter mitral valve-in-valve implantation can be safely performed for dysfunctional bioprosthetic mitral valves in the pediatric age group with favorable early and mid-term outcomes. It can offer a viable alternative in patients with high surgical risk or deemed not fit for conventional surgery. However, we still recommend a long-term study of this approach in a large cohort multicenter study.

ETHICS DECLARATIONS

Conflict of interest: The authors declare that they do not have any conflict of interests.

Ethical Approval: The study was approved by the Institutional Review Board in Prince Sultan Cardiac Center (IRB Number R20034).

Consent for Publication: Not applicable.

\section{How to Prevent Pulmonary Artery Wall Perforation Following Transcatheter Occlusion of Left Atrial Appendix}

Piotr Nikodem Rudziński ${ }^{1}$, Marcin Demkow ${ }^{1}$, Marek Konka ${ }^{1}$, Adam Witkowski ${ }^{1}$, Radosław Pracoń ${ }^{1}$, Piotr Ścisło ${ }^{2}$, Zenon Huczek $^{2}$, Marian Burysz ${ }^{3}$, Wojciech Ogorzeja ${ }^{3}$, Grzegorz Suwalski $^{4}$, Andrzej Kurowski ${ }^{1}$, Cezary Kęka ${ }^{1}$, Anna Klisiewicz ${ }^{1}$, Ilona Michałowska ${ }^{1}$, Zofia Dzielińska ${ }^{1}$, Mariusz Kuśmierczyk ${ }^{1}$

${ }^{1}$ National Institute of Cardiology, Warsaw, Poland. ${ }^{2}$ Medical University of Warsaw, Warsaw, Poland. ${ }^{3}$ Regional Specialist Hospital in Grudziadz, Grudziadz, Poland. ${ }^{4}$ Military Institute of Medicine, Warsaw, Poland

Transcatheter left atrial appendage (LAA) occlusion is an accepted treatment for patients with high risk of thromboembolic events related to nonvalvular atrial fibrillation, who do not tolerate oral anticoagulants. Perforation of the main pulmonary artery (PA) wall by hooks of the devices is a case reported potentially lethal complication. The aim was to identify the "anatomy at risk" to establish the prohibited anchoring zone for the device hooks. We retrospectively analyzed the preimplantation transesophageal echocardiograms (TEE) of the 5 patients (aged 62-74 years) complicated by cardiac tamponade after transcatheter LAA occlusion with the Amplatzer Amulet device. The 
diagnosis of PA wall perforation by the implant stabilizing wires was confirmed in all the patients at rescue surgery, performed from $3 \mathrm{~h}$ to 17 days after the device implantation. All the patients survived. Afterwards, we prospectively analyzed preimplantation TEEs of the 80 consecutive patients scheduled for transcatheter LAA closure in search for the "risky anatomy" for the Amulet. The preimplantation TEE analysis of the 5 patients revealed similar pictures in the $\sim 135^{\circ}$ view. This showed parallel alignment of the LAA and PA walls, directly neighboring each other all along the Amulet landing zone, and where the occluder hooks were supposed to anchor. This "LAAPA $135^{\circ}$ neighbors" situation was recognized in 4 of the 80 following patients. The $\sim 135^{\circ}$ TEE projection identifies the "risky anatomy" for the Amulet hooks anchoring zone. This anatomy is not frequent. The implantation of the Amulet in the "LAA-PA $135^{\circ}$ neighbors" has to be avoided.

\section{ETHICS DECLARATIONS}

Conflict of interest: The authors declare that they do not have any conflict of interests.

Ethical Approval: Not applicable.

Consent for Publication: Not applicable.

\section{Case Report: Aortic Valve Replacement due to Aortic Valve Leaflet Perforation After PCI}

\author{
Yasser Mubarak $^{1}$, Muhammad Wahaab ${ }^{2}$ \\ ${ }^{1}$ Minia University, Minia, Egypt. ${ }^{2}$ Madinah Cardiac Center, Madinah, \\ Saudi Arabia
}

Percutaneous Coronary Intervention (PCI) is widely recognized as an effective treatment for Acute Coronary Syndrome (ACS). In spite of advances in equipment and experience of interventional cardiologist, still there are rare complications occurred. Iatrogenic injury of the aortic valve leaflet is a rare. Aortic insufficiency (AI) after a PCI suggests an iatrogenic valve injury. Aortic leaflet injury is not common but possible complication of PCI. Because of the serious consequences, it should be mentioned in the informed consent. Aortic repair of iatrogenic injury is possible, and it can be performed with excellent clinical and functional midterm results. So, Aortic valve replacement (AVR) is the last option. PCI is a safe technique that has a good result with ischemic heart disease (IHD). However, despite its advantages, it is not risk-free. Acute traumatic (AI) due to catheterization injury is an extremely rare mechanical complication. The mechanism of acute traumatic (AI): (a) transitory regurgitation due to movement of rigid catheters into the aorta arch, and the condition is relieved upon catheter withdrawal (b) irreversible acute AI is extremely rare and is related to stents implanted in the RCA ostium protruding into the aorta (c) direct catheter perforation of aortic valve leaflet, during intervention in the RCA with Amplatz catheters. This type of complication can be prevented by careful catheter handling, especially in situations where catheterization is challenging or in patients with coronary anomalies. Male patient 55 years old known as smoker, hypertensive, diabetic and dyslipidemic presented to our hospital with Non-ST segment Elevation Myocardial Infarction (NonSTEMI). His past history recorded multiple admissions with acute coronary syndrome (ACS) and two PCIs (Stents to Diagonal and RCA in 2015, then Proximal LAD and 1st obtuse marginal in 2019). Transthoracic echocardiogram (TTE) was showed preserved biventricular systolic function without any valvular lesion. Emergency coronary angiogram was performed, due to ongoing chest pain refractory to medical treatment and recent PCI, through radial artery and showed significant distal LM ( Left main),calcified angulated lesion in LAD/CX (Left anterior descending and circumflex), with RCA instent restenosis. He refused CABG and accepted high risk
PCI. Complex PCI was done by multiple trials to engaged left coronary ostia, which failed after multiple trial, and wire was failed to pass through RCA lesion. During the procedure he developed acute heart failure due to recent infarction and arrhythmia. Cardiac markers were elevated before PCI but more elevated Post-PCI failure. Postprocedure TTE showed moderately severe AI with probable aortic valve injury and $\mathrm{EF}=35 \%$. After stabilization at CCU (Coronary Care Unit), trans-esophageal echocardiogram (TEE) was done and showed tear in the NCC with jet of AI (Figs. 1 and 2). We received emergency call from $\mathrm{CCU}$ for $\mathrm{CABG}$ and aortic intervention. Emergency surgery was performed by establishing CPB (CardioPulmonary Bypass) and on pump harvesting LIMA (left internal mammary artery). Then, distal anastomosis of SVG (saphenous vein graft) to RCA, and OM were done. Then, aortotomy was performed, inspection of aortic leaflets revealed tear in NCC and excluded endocarditis (Fig. 3). Aortic valve replacement (AVR) was performed because of extensive cusp tear was beyond repair. Also, the AV repair needs more experience and takes more time which is not suitable in that instability condition. After that, LIMA to LAD and proximal SVG anastomosis were done. IABP (Intra-Aortic Balloon Pump) was inserted with moderate inotropic support was added for easily weaning. Post-operative course was passed uneventful by expert CSICU (Cardiac Surgery Intensive Care Unit).Follow up echo is revealed well function prosthetic aortic valve without gradient, and improvement of EF (45\%) and biventricular function after 6 months. AI caused by a leaflet perforation is seen most frequently with infective endocarditis (IE) involving aortic valve [4]. Iatrogenic aortic valve injury and leaflet perforation have been reported after cardiac operations performed such as repair of a bicuspid aortic valve, aortic

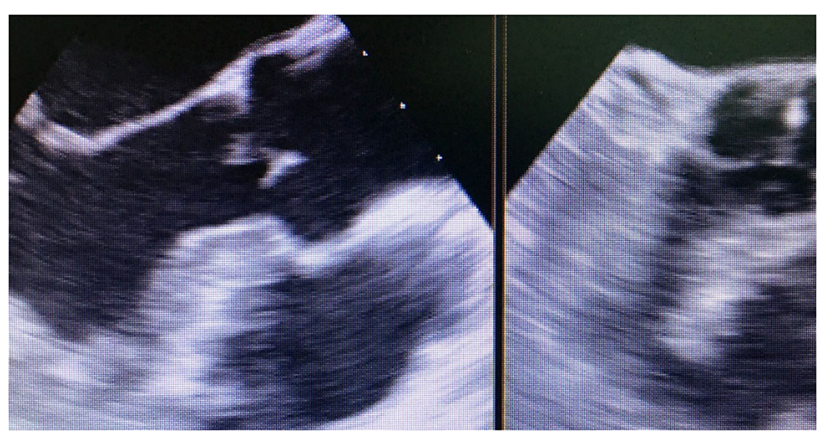

Fig. 1 TEE is showing NCC tear in AV leaflet

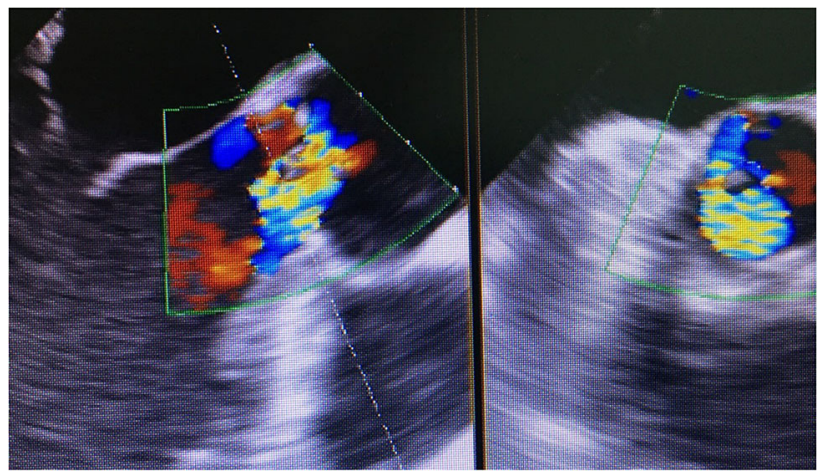

Fig. 2 TEE is showing severe AR, eccentric jet related to NCC 


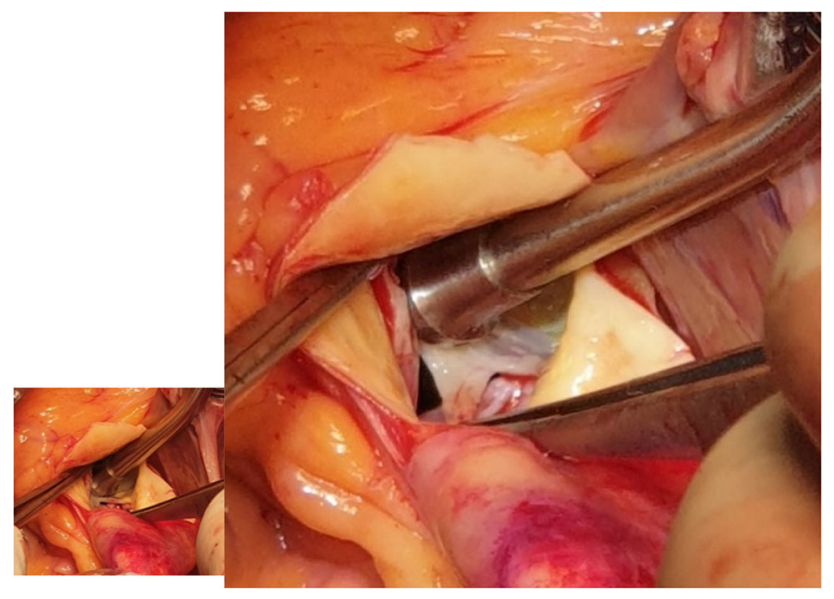

Fig. 3 Intraoperative view is showing tear in NCC

valve decalcification, and septal myectomy [7]. Rarely, suture-related, inadvertent injury to an aortic valve leaflet can produce leaflet tear with regurgitation after cardiac operations performed in the vicinity of the aortic valve (e.g., mitral valve replacement, repair of a membranous ventricular septal defect, and repair of an ostium primum atrial septal defect) [8]. But in our case, previous cardiac surgery and IE was excluded by TEE, Lab., and intraoperative exploration.Iatrogenic $\mathrm{AI}$ is caused by multiple stalling of rotabur in calcified coronary artery caused traction and disengagement of guide causing rotabur to be freely hanged in ascending aorta close to cusps. Very high speed rotablator come in contact with aortic leaflet causing perforation and tear [9]. In our case, rotablator was not used. Although rare post PCI complications (AI, Aortic Dissection, Coronary Artery Dissection); the incidence and clinical implications of these warrants further investigation given the high mortality rate and the growing number of cardiac procedures performed [1]. So, we try to collect all post PCI complications, those need surgical intervention. AI can be caused temporary during Impella implantation due to direct contact with aortic leaflet or permanent after removal due to aortic leaflet injury [5]. In our case, there is not any device used other than PCI. In urgent CABG, LIMA can be safely harvested and hemodynamic instability is no longer a relative contraindication. In standard CABG, LIMA is harvested before establishing CPB. Patients with severe multi-vessel $\mathrm{CAD}$ and tight Left main stem stenosis can become unstable during induction of anesthesia or during surgery. Routine clinical practice in these patients is to establish urgent cardiopulmonary bypass, and only vein conduit is used to bypass all significant coronaries which are easy and rapid to harvest in such condition. So, these patients are deprived of LIMA graft and its long term patency [6]. So, we harvested LIMA after establishing CPB for safety of the patient and because of instability of hemodynamics without deprived patient from long term patency graft to LV (left ventricle) and relative young patient. We performed LIMA on LAD and SVG on OM, which are previously stented because coronary angiography was showed significant distal LM stenosis. So, any cardiac intervention needs surgical backup team who trained well and has experience to deal with rare but lifesaving conditions. Iatrogenic AI is very rare complication of PCI. Aortic valve repair if leaflets tears or perforations are repairable, otherwise, AVR with complete revascularization is safe procedure.

ETHICS DECLARATIONS

Conflict of interest: The authors declare that they do not have any conflict of interests.

Ethical Approval: Not applicable.

Consent for Publication: Not applicable.
015. Early Experience of Pulsta New Self Expandable System for Percutaneous Pulmonary Valve Implantation in The Native Right Ventricular Outflow Tract Dysfunction

Jung Yoon Kim, So Ick Jang, Seong Ho Kim, Ji Seok Bang, Eun Young Choi, Ja Kyoung Yoon, Jung il Noh, Hyun Chung

Sejong General Hospital, Bucheon, Republic of Korea

Percutaneous pulmonary valve implantation (PPVI) is a good treatment option for right ventricular outflow tract (RVOT) dysfunction. This study reports the clinical use in various morphology of RVOT and short-term outcome of a novel transcatheter self-expandable pulmonary valve (Pulsta valve, Teawoong Medical Co, South Korea) in native RVOT dysfunction. A total of 29 patients who underwent PPVI with Pulsta valve in a single center from December 2017 to April 2021 were retrospectively enrolled. Patient demographics and pre-procedural, intra-procedural, and short-term follow-up data were reviewed. The majority of patients were female $(n=19,65.5 \%)$, with a mean age of $28.7 \pm 11.6$ years (range: $14-61$ years) and a mean weight $61.0 \pm 14.3 \mathrm{~kg}$ (range: $39.6-89 \mathrm{~kg}$ ). 28 of 29 Patients showed moderate to severe PR, with a mean PR fraction derived cardiac MRI of $40.8 \pm 10.3 \%$ (range: $11.0-63.0$ ). The procedure success rate was $96.5 \%$ with a mean fluoroscopy time of $25.1 \pm 10.9 \mathrm{~min}$. Valve sizes used were $26(n=3), 28(n=9), 30(n=5), 32(n=7)$, and 33.5 $(n=5)$. Procedural complications were arrhythmia requiring treatment $(\mathrm{n}=2)$, delivery catheter associated complication $(\mathrm{n}=1)$, device embolization $(n=1)$. 1-year follow-up cardiac MRI showed a decreased pulmonary regurgitation $(\mathrm{PR})$ fraction $(12.4 \pm 5.9 \%)$ and that the right ventricular end-diastolic volume index decreased from $168.6 \pm 31.3$ to $128.7 \pm 20.6 \mathrm{ml} / \mathrm{m}^{2}$, the right ventricular end-systolic volume index decreased from $84.1 \pm 21.1$ to $66.5 \pm 14.0 \mathrm{ml} /$ $\mathrm{m}^{2}$. There were no mortality, or re-intervention during follow-up. And no patients had more than mild PR and paravalvular leakage. Pulsta valve provides excellent short-term outcomes in patients with the various morphology of RVOT and extremely large RVOT.

ETHICS DECLARATIONS

Conflict of interest: The authors declare that they do not have any conflict of interests.

Ethical Approval: Not applicable.

Consent for Publication: Not applicable.

\section{Closure of a Superior Vena Cava to Right Upper Pulmonary Vein Window with Two Covered Stents}

\author{
Abraham Rothman ${ }^{1,2}$, Alvaro Galindo ${ }^{1,2}$, William Evans ${ }^{1,2}$, \\ Humberto Restrepo 1,2 \\ ${ }^{1}$ Children's Heart Center Nevada, Las Vegas, USA. ${ }^{2}$ University \\ of Nevada Las Vegas, Las Vegas, USA
}

A 66-year-old previously athletic male with atrial fibrillation was undergoing a transvenous pacemaker for tachy-brady arrhythmia at another institution. The pacemaker lead ended in the left ventricle. The pacemaker lead was removed and the patient had a CT scan. It suggested a sinus venosus defect with partial anomalous venous return of the right upper pulmonary vein. A cardiac catheterization showed a $\mathrm{Qp} / \mathrm{Qs}>2$ and mild elevation of pulmonary arterial pressures. He was referred to our center. Review of the CT images and echocardiogram were not consistent with the diagnosis. Reconstruction of the CT images and axial views showed a superior vena cava (SVC) to right upper pulmonary vein (RUPV) window (Fig. 1A and $\mathrm{B})$, with the RUPV retaining normal connection with the left atrium. 
At cardiac catheterization, the $\mathrm{Qp} / \mathrm{Qs}$ was $1.5 / 1.0$, the mean pulmonary arterial pressure was $21 \mathrm{mmHg}$ and the pulmonary vascular resistance was 2.8 W.U. There was a large SVC-RUPV window. The SVC dimension was $17-18 \mathrm{~mm}$ proximally and $30-32 \mathrm{~mm}$ distally (near the junction with the right atrium) (Fig. 2A). Through an $18 \mathrm{~F}$ right femoral venous sheath, a $32 \mathrm{~mm} \times 5 \mathrm{~cm}$ Endurant II aortic graft extension was delivered in the distal SVC. A $4.5 \mathrm{~cm}$ covered CP stent was delivered telescoped into the proximal end (site of stabilizing hooks) of the Endurant graft, utilizing a $20 \mathrm{~mm}$ balloon (Fig. 2B). An angiogram (performed with a catheter advanced through a patent foramen ovale) and TEE showed no obstruction of RUPV return to the left atrium (Fig. 3A and B). Two weeks after the procedure, the patient was doing well clinically. A transthoracic echocardiogram showed patent SVC stents and good biventricular function.

Figure abbreviations: $\mathrm{AO}=$ aorta, $\mathrm{LA}=$ left atrium, $\mathrm{LUPV}=$ left upper pulmonary vein, $\mathrm{SVC}=$ superior vena cava.

\section{ETHICS DECLARATIONS}

Conflict of interest: The authors declare that they do not have any conflict of interests.

Ethical Approval: This case report is exempt of IRB Approval.

Consent for Publication: Not applicable.
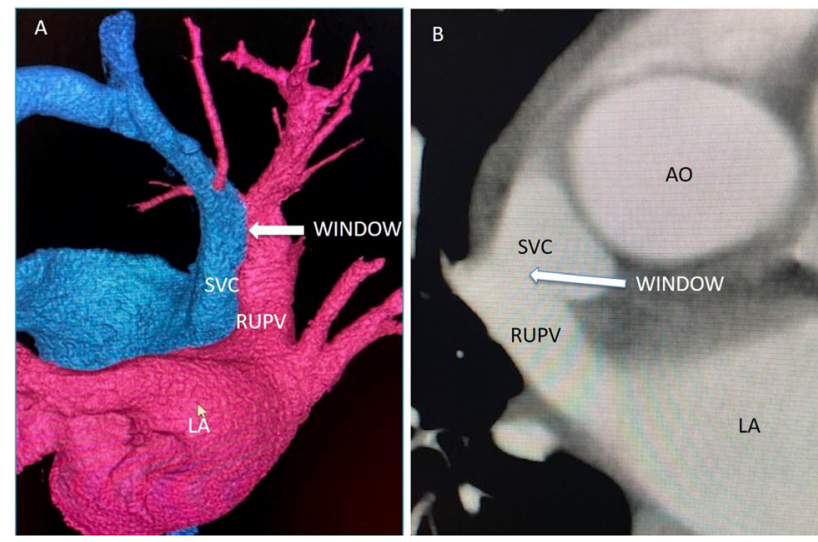

Fig. 1 .
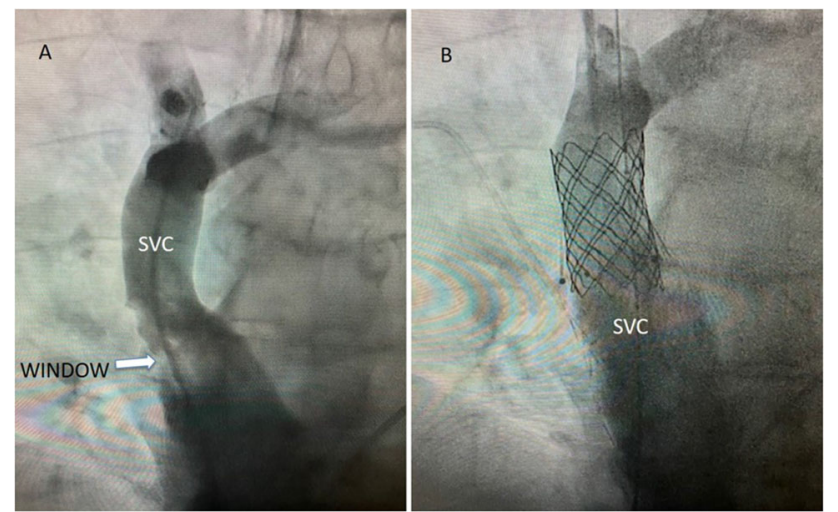

Fig. 2 .

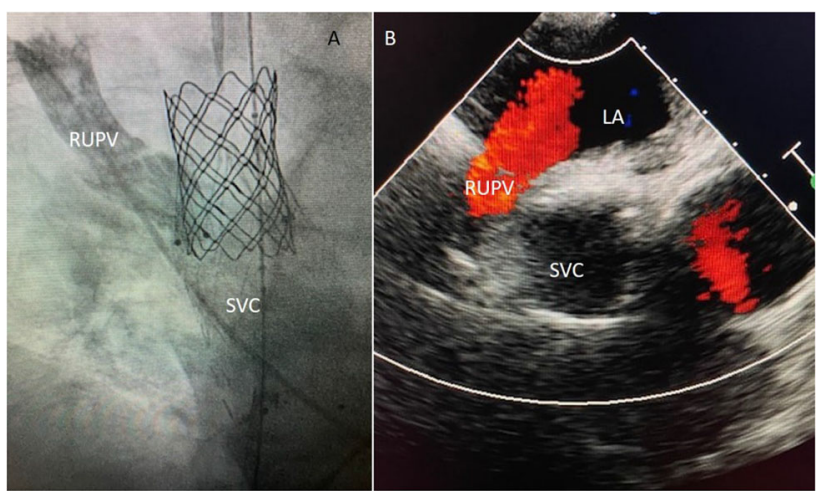

Fig. 3 .

\section{Transcatheter Repair of Coronary Sinus ASD and Persistent Left Superior Cava: First Case Report in an Infant and Review of the Literature}

\section{Michael Vance, MD}

Children's Hospital of the King's Daughters, Norfolk, VA, USA

Coronary sinus atrial septal defects (CSASDs) are rare entities that result from partial or complete unroofing of the coronary sinus. When CSASDs occur in isolation they can result in significant left to right shunt. When CSASDs are present with a persistent left superior vena cava (PLSVC) there are added risks of cyanosis, TIA, stroke and cerebral abscess secondary to right to left shunt. Surgery for CSASDs with PLSVC frequently consists of closure of the coronary sinus ostium (CSO) and ligation of the PLSVC when there is an adequate bridging vein. Interventionalists have emulated the surgical approach through device closure of the CSO and PLSVC in adult patients. Whether accomplished by surgical or interventional means, this approach intentionally leaves a communication between the coronary sinus and left atrium, potentially resulting in mild systemic oxygen desaturation. However, no desaturation has been reported on followup of CSASD patients who have undergone CSO device closure. We report the first case of device closure of CSO and PLSVC in an infant with a CSASD and PLSVC with a bridging vein. The case illustrates the importance of understanding coronary venous anatomy when placing devices in the CSO. We also review previous reports and offer a hypothesis to explain the absence of systemic oxygen desaturation in our and other patients. Case Report: An 11 month old female was found by echocardiography to have a type III CSASD and PLSVC with bidirectional shunt. At cardiac catheterization Qp:Qs was 1.5:1. The CSO and LSVC measured $4 \mathrm{~mm}$. A $4 \mathrm{~mm}$ Amplatzer VSD Occluder was delivered across the CSO after defining the location of the middle cardiac vein. A $5 \mathrm{~mm}$ Amplatzer plug 4 was delivered into the PLSVC. At follow-up the child had no residual coronary sinus ostium or PLSVC flow. She had normal systemic oxygen saturation and was in normal sinus rhythm.Review of the literature and discussion: Device closure of the CSO has previously been reported in 11 adult patients with CSASDs. 10 patients received Amplatzer Septal Occluders and 1 received an Amplatzer plug 2. All of the cases were successful. None had residual shunt, conduction disturbance or systemic desaturation on follow-up. The absence of desaturation in our and other reported patients can be explained by the known communications between the coronary sinus tributaries and the noncoronary sinus tributaries and Thebesian vessels. Just as atrial septal defects result in left to right shunt, coronary venous blood that has alternative communication with the left and right atrium will follow 
the latter pathway. However, it is important to note that despite these communications acute occlusion of important coronary veins can lead to cardiac dysfunction and therefore an understanding of the coronary venous system is necessary when performing interventions in the coronary sinus. Adult and pediatric patients with CSASDs can safely undergo device closure of the CSO. Despite the residual communication between the coronary sinus and the left atrium following this intervention, systemic oxygen desaturation has not been observed in patients on follow-up. This may be explained by the extensive communications between, and variable drainage of, the cardiac veins. An understanding of the coronary venous anatomy is necessary for safe intervention in the coronary sinus.

ETHICS DECLARATIONS

Conflict of interest: The authors declare that they do not have any conflict of interests.

Ethical Approval: Not applicable.

Consent for Publication: Not applicable.

\section{Late Presentation of Asymptomatic Pediatric Patient with Anomalous Left Coronary Artery}

\section{Nora Elsamman, Nevin Habeeb}

\section{Children's Hospital, AinShams University, Cairo, Egypt}

This case describes the late presentation of an asymptomatic 7 years old ALCAPA patient. The patient was accidentally discovered during routine echocardiographic follow up for multiple muscular ventricular septal defects, which turned out to be an operator misinterpretation of dilated tortuous coronary artery septal branch. He had average ventricular dimensions with good global ventricular function, mild left atrium dilatation, no ventricular septal defects, and moderate mitral regurgitation. Meanwhile, the posterior papillary muscle appeared to be echogenic and sclerosed. There was a tortuous dilated vessel flow through the ventricular septal wall. In the short axis view, the right coronary artery had a prominent course being divided into two main branches, while the left coronary artery could not be visualized. Left ventricle speckle tracking showed normal global longitudinal strain (GLS $=-16.5 \%)$, meanwhile. The posteromedial regions (near the sclerosed posterior papillary muscle) showed decreased function ( -6.5 and $9.5 \%$ ) denoting decreased perfusion to these regions. To further visualize the left coronary artery, multislice cardiac CT was performed which showed that the left main coronary artery has an anomalous origin from Latero-inferior aspect of proximal main pulmonary artery, bifurcating into left anterior descending and nondominant left circumflex. While the right coronary artery is dominant, arising from right coronary sinus and running through the septum as posterior descending artery. Cardiac catheterization was done for better demonstration of the coronary arteries anatomy. Aortic root angiogram showed dilated right coronary artery giving rise to dilated posterior descending artery which gives extensive collaterals supplying the left ventricular myocardium with significant steal and filling the pulmonary artery through collaterals connected to very small rudimentary left coronary artery which arises from the main pulmonary artery. Main Pulmonary artery angiography showed a very small rudimentary stump arising from the inferior aspect of the pulmonary artery. The patient had longstanding ischaemic myocardium with preserved global ventricular function. The patient has been compensated hemodynamically for seven years with moderate mitral regurge due to sclerosed papillary muscle. There is no management guidelines in our patient, so we aimed to discuss the challenge of determining the need for intervention; the benefits of surgical revascularization are to be considered for the risks of cardiopulmonary bypass on scarred left ventricle myocardium versus the benefits of repair and potential reversal of ongoing myocardial dysfunction. Meanwhile, transcatheter technique can offer a new approach by occlusion of the pulmonary artery, which may be still controversial.

ETHICS DECLARATIONS

Conflict of interest: The authors declare that they do not have any conflict of interests.

Ethical Approval: Not applicable.

Consent for Publication: Not applicable.

\section{Congenital Ventricular Aneurysm: A Difficult Diagnosis to Make.}

\author{
Nora Elsamman $^{1}$, Nevin Habeeb ${ }^{2}$ \\ ${ }^{1}$ Childrens Hospital, Ainshams University, Cairo, Egypt. ${ }^{2}$ Childrens \\ Hospital, Ainshams University, Cairo, Egypt
}

A pouch arising from the left ventricle wall may be a congenital ventricular diverticulum, pseudoaneurysm or an aneurysm. Each has distinct presentation, association, morphology and prognosis. Identification of each is important for treatment decisions. Aneurysm is an outpouching containing all the histologic components of the tissue wall. It can be seen in all cardiac chambers, most commonly in the left ventricle. It may be dyskinetic due to the predominance of fibrous tissue without organized myocardium. They are almost always associated with a wide communication to the ventricle and are not associated with other congenital anomalies. We describe a five years old girl who was presented to our hospital for routine Echo follow up as a known case of ventricular septal defect. On physical exam, the child had normal saturations on room air and a 3/6 systolic murmur heard best at the left lower sternal border. Chest X-ray revealed cardiomegaly. Electrocardiogram showed normal heart rate and rhythm. No electrocardiogram abnormalities were detected. Initial cardiac echocardiography evaluation revealed dilated left atrium and left ventricle with a large apical aneurysm. Cardiac MRI demonstrated a large post-infarction like ventricular septal defect with normal coronary arteries. Thrombophilia profile was done for exclusion of causes of coronary artery disease causing myocardial infarction. Cardiac hemodynamic study showed normal pulmonary artery pressure, so the decision was made to close the defect by transcatheter approach. Congenital ventricular aneurysms are rare, yet potentially fatal anomalies. Being aware of this rare anomaly is crucial for the diagnosis.

ETHICS DECLARATIONS

Conflict of interest: The authors declare that they do not have any conflict of interests.

Ethical Approval: Not applicable.

Consent for Publication: Not applicable.

\section{Left Ventricle Non-compaction Cardiomyopathy with Right to Left Shunt: Close or Not to Close}

\section{Nora Elsamman, Nevin Habeeb}

Children's Hospital, AinShams University, Cairo, Egypt

Left ventricular cardiomyopathy can be presented in variable ways, including accidental discovery during cardiac imaging in asymptomatic patients to symptomatic heart failure, cardiac arrythmia or sudden death. Congestive heart failure can be due to systolic or diastolic dysfunction. Trabeculations decrease ventricular compliance and cardiac muscle relaxation leading to diastolic dysfunction. In our 
case we describe an eleven years old girl with late onset cyanosis at the age of 10 years. Cardiac imaging showed that she suffered from dilated left ventricle with non-compaction cardiomyopathy, atrial septal defect with bidirectional flow across and dilated inferior venae cava. The systolic function was borderline, while the diastolic function was impaired. Meanwhile, she had normal pulmonary artery pressure. Cardiac catheterization proved normal pulmonary venous drainage and absence of pulmonary arterio-venous communication. The condition was progressive with deepening of cyanosis with increased exercise intolerance. Echocardiogram showed increased right to left shunt across the atrial septal defect. The decision was made to close the defect by trans-catheter approach with a challenge of having a risk of impaired right ventricular function. After which, the patient saturation raised to normal and the cardiac function was markedly improved post-operatively. We discuss left ventricular noncompaction cardiomyopathy diastolic dysfunction in the presence of right to left cardiac shunt and the controversy of cardiac intervention.

\section{ETHICS DECLARATIONS}

Conflict of interest: The authors declare that they do not have any conflict of interests.

Ethical Approval: Not applicable.

Consent for Publication: Not applicable.

\section{Can Duct Stenting be a Rescue Beyond the Neonatal Period}

\section{Nora Elsamman, Nevin Habeeb}

Children's Hospital, AinShams University, Cairo, Egypt

Infants with various forms of congenital heart disease that include ductal-dependent pulmonary blood flow often require an early palliative procedure to augment or establish a reliable source of pulmonary blood flow. Stent implantation into the arterial duct has been proposed as a nonsurgical alternative to aortopulmonary shunt surgery. It offers the potential advantage of eliminating the need for palliative surgery, thus reducing the number of operations required and optimizing the time of definitive surgical correction. The problems in developing countries, especially Egypt, are due to the large number of patients with small pulmonary arteries to shunt, a long list of waiting patients, limited centers for pediatric cardiac surgery, and the majority of patients with critical conditions are not suitable for surgery. Another issue is that the cost of PDA stenting is much less than the cost of an aortopulmonary shunt including an intensive care admission and a long hospital stay. Our case is a 4 months old baby with deep cyanosis recognized at the age of 4 months. Echocardiography confirmed the diagnosis of duct dependent pulmonary circulation with a small tortuous PDA less than $2 \mathrm{~mm}$ diameter with faint flow across. Prostaglandin E1 infusion was started immediately. Oxygen saturation improved to $45 \%$. No apneic episodes were experienced. Surgical intervention was refused by the surgeons due to the bad general condition of the baby. So, the baby was taken up for ductal stenting. Coronary stent with diameter $3.5 \mathrm{~mm}$ and length of $20 \mathrm{~mm}$, premounted on low-profile balloon dilation catheter was inflated in the duct after making sure that the full length of duct including both ends is adequately covered by the stent. His saturation raised to $85-90 \%$. The baby was transferred to the intensive care unit. There was fear of desaturation on probing the duct especially that the prostaglandin wouldn't be much effective. However, the procedure went smoothly. In conclusion, duct stenting can be considered as a rescue beyond the neonatal age.
ETHICS DECLARATIONS

Conflict of interest: The authors declare that they do not have any conflict of interests.

Ethical Approval: Not applicable.

Consent for Publication: Not applicable.

\section{Opening a Closed Arterious Duct and Saving a Pulmonary Branch in a Complex Fallot's Anatomy}

\author{
Marco Ruiz-Ontiveros, Alejandro Flores-Arizmendi, Jesus \\ Montalvo-Aguilar, Antonio Salgado-Sandoval
}

National Medical Center "20 de noviembre” ISSSTE, Mexico City, Mexico

Tetralogy of Fallot is the most common cyanotic congenital heart disease, however we can still find rare associations within its spectrum. We present a case where, apart from the unusual anatomy, there was evidence of the existence of the left pulmonary branch but without continuity of the pulmonary trunk and with a closed ductus arteriosus that was resolved with a known therapeutic option and applicable in this type of cases. A 9-month-old woman referred with diagnosis of tetralogy of Fallot. The echocardiogram corroborates the diagnosis, in addition to the absence of the left pulmonary branch, right aortic arch and subaortic innominate vein, this association found in less than $1 \%$ of cases. Cardiac catheterization was performed where the diagnosis was confirmed where only the main and right pulmonary arteries were observed, the Singh maneuver was performed, with retrograde injection in the left superior pulmonary vein, the existence of a left pulmonary branch was evidenced, which was of adequate size. In the aortic arch without evidence of ductus arteriosus, however, a ductal ampulla was observed without the pass of contrast medium, it was attempted and it was cannulated with a coronary guide towards the pulmonary branch, which showed that there was continuity. Due to the position of the duct, it was decided to access through the right carotid with a JR 6Fr catheter and coronary guide until it was placed in the lower left lobar, subsequently a plasty was performed at the ductus site with a $2.5 \times 20 \mathrm{~mm}$ balloon. Then a $4.5 \times 16 \mathrm{~mm}$ coronary stent was placed at the level of the ductus, achieving patency towards the left pulmonary branch. Hours after the procedure, the patient presented findings of reperfusion in the left lung, which was managed with ventilatory maneuvers and drugs in the PICU. Forty-eight hours later in echocardiographic and tomographic control, a decrease in the stent's lumen was evidenced, so it was decided to perform a new catheterization. Ultrasound-guided right carotid access was again obtained, with a JR 5 Fr catheter the coronary guide was placed in the lower left lobar, a $4.5 \times 16 \mathrm{~mm}$ stent was placed inside the previous one, then we made a plasty of the stent with a $5 \times 15 \mathrm{~mm}$ coronary balloon, material was removed and performed control angiography with evidence of adequate flow through the ductus arteriosus to the left pulmonary branch. The patient evolved adequately and was discharged within a week. The echocardiogram prior to discharge confirmed patency of the stent. He was discharged with medical management with ASA and clopidogrel. Ductal stent placement is a common practice in complex heart diseases with pulmonary flow compromise, however the cases in which a closed ductus arteriosus are open with an interventional procedure are less frequent and generally occur in the first months of life. We share a case of successful opening and stenting in a closed ductus arteriosus, with recovery of the ipsilateral pulmonary branch, in a rare anatomy of Tetralogy of Fallot, which totally changed the prognosis of this patient. 


\section{ETHICS DECLARATIONS}

Conflict of interest: The authors declare that they do not have any conflict of interests.

Ethical Approval: Not applicable.

Consent for Publication: Not applicable.

023. Transcatheter Mitral Valve-in-Valve Implantation in a Patient with Congenital Mitral Stenosis and Early Surgically Implanted Valve Failure

\section{Jose Antonio Cornejo-Guerra, Guering Eid Lidt, Jose Antonio Garcia-Montes, Randy Richard Querevalu, Edgar Lupinta- Paredes, Sergio Tolento Peraza, Andrea Castillo, Roberto Dawson, Juan Pablo Sandoval-Jones}

Instituto Nacional De Cardiologia Ignacio Chavez, Mexico City, Mexico

Currently, transcatheter mitral valve-in-valve (MViV) using balloonexpandable transcatheter valves has emerged as an attractive alternative for patients deemed with high-surgical risk. In adults with congenital heart disease (ACHD), MViV may be offered to delay reoperation or as an alternative to a mechanical prosthesis, particularly in young adults to avoid lifelong anticoagulation. We present the case of an 18-year-old female diagnosed with congenital mitral stenosis due to true parachute mitral valve. She underwent mitral plasty at 14 years of age. Significant mitral regurgitation compelled replacement with a $25 \mathrm{~mm}$ Magna Ease ${ }^{\circledR}$ (Edwards) bioprosthetic valve. She developed left heart failure symptoms including dyspnea and significantly decreased exercise tolerance within a relatively short period of time after surgery. The echocardiogram revealed severe stenosis of the bioprosthetic valve (mean echocardiographic gradient of $16 \mathrm{mmHg}$ ) and moderate tricuspid regurgitation. A consensus between the patient and her family and the heart team to pursue MViV was made provided she refused a mechanical valve option due to concerns with lifelong anticoagulation and future childbearing desire. Preprocedural cardiac CT scan showed an effective Internal diameter of $25 \mathrm{~mm}$ with a $2.42 \mathrm{~cm}^{2}$ ring area ( $77 \mathrm{~mm}$ perimeter). The virtual neo-Left ventricle outflow tract was estimated in $2.45 \mathrm{~cm}^{2}$. Cardiac catheterization was performed under general anesthesia. Intracardiac echo guided transseptal puncture was ultimately performed using an electro-cut assisted-BKN transseptal needle due to a challenging interatrial septum anatomy and prior unsuccessful

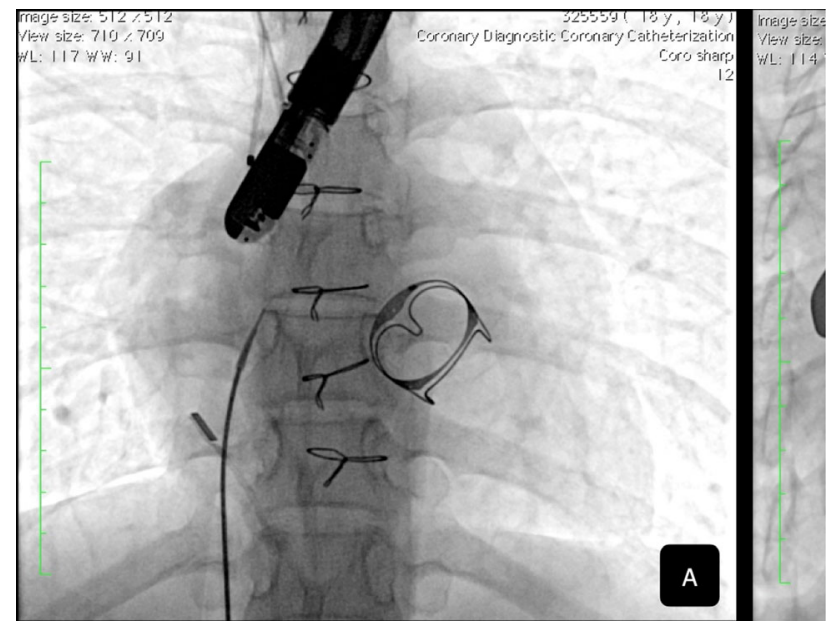

Fig. 1 . attempts. (Fig. 1A). Crossing into the ventricle was facilitated by an Agilis NxT® (Abbott cardiovascular) steerable introducer to allow placement of a Safari ${ }^{\circledR}$ guidewire (Boston Scientific, USA) into the apex of the left ventricle (LV). The valve was pre-dilatated with a $25 \mathrm{~mm}$ Maxi LD® (Cordis, Johnson \& Johnson, USA). Controlled deployment of a $26 \mathrm{~mm}$ Sapien3 ${ }^{\circledR}$ Valve (Edwards Lifesciences, USA) was implanted under rapid pacing (Fig. 1B). Final hemodynamics revealed mean left atrium (LA) pressure of $6 \mathrm{mmHg}$ (baseline mean LA pressure $17 \mathrm{mmHg}$ ) and a LA-to-LV gradient of $3 \mathrm{mmHg}$, without any left ventricular outflow obstruction. No mitral regurgitation was seen on echo. Patient was successfully discharged $48 \mathrm{~h}$ later. Dual antiplatelet therapy was prescribed. Transcatheter MViV implantation offers a feasible and safe option in select patient with ACHD. Long-term outcome of MViV in this population remains yet to be determined.

ETHICS DECLARATIONS

Conflict of interest: The authors declare that they do not have any conflict of interests.

Ethical Approval: Review board approved the manuscript.

Consent for Publication: Not applicable.

\section{Interventional Cardiac Catheterization Procedures in Pediatric Cardiac Transplant Patients}

Fernando Ballesteros ${ }^{1}$, Alejandro Rodriguez Ogando ${ }^{2}$, Manuela Camino Lopez ${ }^{1}$, Nuria Gil Villanueva ${ }^{1}$, Juan Miguel Gil Jaurena $^{1}$, Ricardo Sanz Ruiz ${ }^{1}$, Jose Luis Zunzunegui ${ }^{1}$

${ }^{1}$ Gregorio Marañón Hospital, Madrid, Spain. ${ }^{2}$ Gregorio Marañón Hospital., Madrid, Spain

The indications for performing orthotopic heart transplant (OHT) in the pediatric population include cardiomyopathy, congenital heart disease not suitable for repair or palliation, and failed repairs or palliations. Patients in the latter two categories inherently have complex anatomies and frequently have great vessel abnormalities and unusual venous and arterial connections. New or residual lesions are thus not unexpected following cardiac transplantation. The objectives of this study are to report the spectrum of cardiac lesions in pediatric patients post-OHT, the characteristics of patients who develop these lesions, and the feasibility of transcatheter intervention in treating these lesions. Data was collected through retrospective review of all OHT patients under 18 years, performed at our institution from 1994 to 2020. During the study period, 140 heart transplants were performed. Twenty-two patients, age 8.1 [0.3-17] years, with a weight of 26.5 [4-90] kg, underwent 31 interventional procedures. 14 patients $(63 \%)$ with original diagnoses of hypoplastic left heart syndrome and failed/or hybrid palliations required intervention most frequently; only 5 patients $(16.5 \%)$ with primary cardiomyopathy were treated. Median time from transplant to intervention was 0.6 months (1 day to 12 years), with $70 \%$ of the procedures performed within the first two months after transplantation. Six types of percutaneous interventions were performed: (A) 9 patients received Systemic suture vein angioplasty, including Superior Vena Cava stent placement in 6 cases. (B) 6 patients received treatment of branch pulmonary artery, with stent implantation in two cases. (C) Aortic arch obstruction was treated in 5 patients, including two Hybrid procedures for stent implantation in the ascendent Aorta suture line. (D) Ilio-femoral vein occlusion was treated with balloon angioplasty and stent placement in 3 patients to achieve vessel patency. (E) Occlusion of aortopulmonary collaterals were performed in 5 patients, including 1 case of life-threatening airway bleeding successfully resolved. (F) 3 patients with cardiac allograft vasculopathy received percutaneous coronary interventions. Major 
complications included two cases of vascular rupture after vein angioplasty performed in the immediate postoperative period, one requiring emergent surgical treatment. Cardiac catheterization is an essential component of care in pediatric orthotopic heart transplant patients. Patients with original diagnoses of Hypoplastic left heart syndrome are at special risk for development of post-transplant anastomotic and vascular obstructive lesions. These lesions can be successfully addressed using interventional techniques; their diagnosis should be aggressively sought out as part of routine post-transplant care.

\section{ETHICS DECLARATIONS}

Conflict of interest: The authors declare that they do not have any conflict of interests.

Ethical Approval: Not applicable.

Consent for Publication: Not applicable.

\section{Transcatheter Suture Closure of PFOs in Patients with Device Closure Contraindications}

\section{Neha Ahluwalia, Robert Pass, Barry Love}

\section{Mount Sinai Hospital, Icahn School of Medicine, New York, USA}

Randomized trials have demonstrated benefit to PFO device closure over medical therapy in prevention of PFO-related stroke. One contraindication to device implantation is bacteremia where there exists a significant risk of seeding the implant leading to endocarditis. We describe two patients with stroke attributed to a PFO with relative contraindication to the use of an occluder device due to recurrent bacteremia. In lieu of device, a NobleStitch ${ }^{\mathrm{TM}}$ EL suture-based closure system was used to close the PFO. Case 1: 65 y.o. male with gastric cancer and multiple surgeries with biliary leak and recurrent bacteremias. He suffered a right parietal occipital stroke attributed to a paradoxical embolism through a PFO in the setting of a left brachial DVT. PFO closure was indicated to prevent recurrent stroke prior to listing for liver transplantation. Case 2: 33 y.o female with sickle cell disease on chronic transfusion program, end stage renal failure and recurrent bacteremias presented with aphasia and difficulty ambulating. Evaluation demonstrated multifocal strokes. TEE showed a tunneled dialysis catheter with debris and a PFO with resting right-toleft shunt. Blood culture positive for Streptococcus oralis. Her HbS fraction was low and the stroke was thought to be due to paradoxical embolism of debris on the indwelling catheter which was removed. However the patient required a new hemodialysis catheter. Both patients were deemed to be high-risk for recurrent stroke from paradoxical embolization. Standard device-based closure was deemed to be contraindicated due to recurrent bacteremia and the risk of device-associated endocarditis. PFO closure with a suture-based system was felt to be the best option in these high risk patients. The procedures were performed under general anesthesia via the right femoral vein. TEE was used for identifying the shunt across the PFO pre and post procedure. Both patients had significant shunt by bubble study prior to the procedure. The PFOs were suture-closed using the NobleStitch ${ }^{\mathrm{TM}}$ EL system (HeartStitch, Inc.) Post-stitch TEE showed no residual bubble shunt in both cases. There were no acute complications related to the procedure. Patient \#2 had placement of new hemodialysis catheter two days later. In short-term follow-up neither patient has had recurrent bacteremia or recurrent stroke. Transcatheter suture closure of PFO was feasible in 2 high risk patients that had relative contraindication to device-closure due to the risk of endocarditis. Suture-based PFO closure may be the preferred method of PFO closure in patients who have device contraindications. A prospective clinical trial (STITCH) to study the efficacy of the NobleStitch ${ }^{\mathrm{TM}} \mathrm{EL}$ closure procedure in comparison with the
Amplatzer Occluder is underway. Results of this trial will clarify the efficacy and safety of this device in comparison with more standard approaches to PFO closure.

\section{ETHICS DECLARATIONS}

Conflict of interest: The authors declare that they do not have any conflict of interests.

Ethical Approval: Not applicable.

Consent for Publication: Not applicable.

\section{Stenting of the Interatrial Septum for Left Atrial Decompression in a Patient with Severe Left Heart Failure}

Randy Richard Querevalu-Barba, E Lupinta, R Dawson, JP Sandoval, JA Garcia-Montes, JA Cornejo

Instituto Nacional De Cardiologia Ignacio Chavez, Mexico City, Mexico

Balloon atrial septostomy (BAS) represents an effective palliative option for patients with severe pulmonary arterial hypertension and heart failure with preserved ejection fraction (HFpEF), also referred to as diastolic heart failure. One of BAS's well-known limitations involves durable shunt patency, with a significant proportion of shunts closing months after creation. Atrial flow regulators have shown promising results in preserving shunt patency. Still, widespread implantation remains somewhat limited to few centers, and cost remains a considerable drawback, particularly in low-to-middle-income countries like ours. Atrial septal stenting remains an attractive option trying to provide a controlled and more durable shunt. We present the case of an symptomatic 11-year-old patient with severe aortic valve stenosis and a non-apex forming left ventricle with diastolic dysfunction. In addition, a large patent ductus arteriosus (PDA) was documented with moderate to severe pulmonary hypertension. We performed a right and left heart catheterization. Severe pulmonary hypertension was determined to belong to Group 5 (mixed pattern) with both precapillary (Group 1) involvement due to the large PDA and obstructive left heart (Group 2) disease. Balloon aortic valvuloplasty was performed using a $16 \mathrm{~mm}$ balloon. The aortic valve gradient fell from 60 to $18 \mathrm{mmHg}$ with mild-to-moderate aortic regurgitation. The decision to close the PDA was conducted in the interest of relief an already impaired left ventricle from significant volume overload, and a 16/18 mm Cera PDA Occluder (Lifetech Scientific LTD) was used to close the duct. Six months later, the patient was admitted with decompensated heart failure and left atrial hypertension and started aggressive IV therapy. Repeat cardiac catheterization revealed biventricular failure with $>20 \mathrm{mmHg}$ left ventricular end-diastolic pressure. Sequential static balloon atrial septostomy was performed with considerable clinical improvement. One month later, we decided to place a stent in the interatrial septum (IAS) to extend shunt patency. Under fluoro and intracardiac echo guidance, a $4 \mathrm{~mm}$ shunt was noticed in the IAS with predominantly left-to-right shunting. A $6 \times 18 \mathrm{~mm}$ Herculink (Abbott Cardiovascular, USA) was initially placed in the IAS. However, the stent soon lost stability and embolized into the left atrium. Snaring and retrieving the stent was achieved uneventfully. We then decided to use a bigger (and stiffer) $8 \times 17 \mathrm{~mm}$ Express LD stent ${ }^{\mathrm{TM}}$ (Boston Scientific, USA). A stable and balanced stent position was obtained with both proximal and distal ends flared to get a "butterfly shape" configuration. A left-to-right shunt was seen on echo (vena contracta $6 \mathrm{~mm}$ ) with no gradient between the left and right atrium $(13 \mathrm{mmHg})$. The patient was discharged with improved clinical status and remains under close surveillance at the heart failure outpatient clinic. 
ETHICS DECLARATIONS

Conflict of interest: The authors declare that they do not have any conflict of interests.

Ethical Approval: Not applicable.

Consent for Publication: Not applicable.

\section{Mid-Term Outcomes from the Harmony Transcatheter Pulmonary Valve Pivotal Trial and Continued Access Study}

\author{
Matthew Gillespie ${ }^{1}$, Doff McElhinney ${ }^{2}$, Thomas Jones ${ }^{3}$, Daniel \\ Levi $^{4}$, Shicheng Weng ${ }^{5}$, John Cheatham ${ }^{6}$ \\ ${ }^{1}$ Children's Hospital of Philadelphia, University of Pennsylvania, \\ Philadelphia, PA, USA. ${ }^{2}$ Lucille Packard Children's Hospital, \\ Stanford University School of Medicine, Palo Alto, CA, USA. \\ ${ }^{3}$ Seattle Children's Hospital, Seattle, WA, USA. ${ }^{4}$ UCLA Mattel \\ Children's Hospital, Los Angeles, CA, USA. ${ }^{5}$ Medtronic, Mounds \\ View, MN, USA. ${ }^{6}$ Nationwide Children's Hospital, Columbus, OH, \\ USA
}

Harmony transcatheter pulmonary valve (TPV) implantation is a less invasive alternative to surgical pulmonary valve replacement in patients with a patched or native right ventricular outflow tract and severe pulmonary regurgitation (PR). The Harmony Pivotal trial is a prospective, non-randomized study with 12 participating sites in the US, Canada, and Japan. Key inclusion criteria were (1) the presence of pulmonary regurgitation by either: severe PR on echocardiogram, or PR fraction $\geq 30 \%$ by magnetic resonance imaging, and (2) an accepted clinical indication for pulmonary valve replacement. The primary safety endpoint is freedom from procedure- or device-related mortality at 30 days. The primary effectiveness endpoint is the percentage of patients with acceptable hemodynamic function at 6 months (mean RVOT gradient $\leq 40 \mathrm{mmHg}$ AND PR fraction $<20 \%$ by CMR or $<$ moderate PR by core laboratory echo review) and no Harmony valve intervention. A Continued Access Study (CAS) following the same protocol as the Harmony Pivotal trial is underway and is the subject of this presentation. A total of 68 patients from Pivotal $(\mathrm{N}=31)$ and CAS $(\mathrm{N}=37)$ were catheterized and 67 were implanted. The median age at catheterization was 29 years (Q1, Q3: 19, 39), and most patients (87\%) had previously repaired tetralogy of Fallot. In the Pivotal Trial, 21 patients received a TPV22 device and 10 received a TPV25. In the CAS, 1 patient received a TPV22 and 35 received a TPV25. This presentation will report full 6-month outcomes for both CAS and the Pivotal Trial. Recently, we reported 30-day data from CAS and 12-month data from the Pivotal trial that demonstrated no explants, endocarditis, major stent fractures, thrombosis, or death and over $90 \%$ of patients with no or trace pulmonary regurgitation at all follow-up visits. Previously reported data from the Harmony Pivotal Trial, including 30-day results from CAS have demonstrated favorable safety and hemodynamic performance of the Harmony TPV. Additional follow-up through 6 months from the CAS will provide important longer-term data on a larger subset of patients with regard to the safety and durability of the Harmony TPV.

\section{ETHICS DECLARATIONS}

Conflict of interest: Matthew J. Gillespie: consultant for Medtronic; on the advisory board and as a consultant for Abbott; and as a consultant and principal investigator for W. L. Gore and Associates.
Doff B. McElhinney: investigator, proctor and consultant for Medtronic.

Thomas K. Jones: investigator, proctor and consultant for Medtronic; Edwards: Investigator, Consultant; W.L. Gore: Investigator, Consultant; Abbott: Investigator, Consultant, Proctor; atHeart Medical: Investigator, Consultant;Daniel S. Levi: consultant for Edwards and Medtronic.

Shicheng Weng: employee and shareholder of Medtronic.

John P. Cheatham: consultant, as a principal investigator, as a proctor, and on the advisory board for Medtronic. Beijing Med-Zenith Medical Scientific Co. LTD: Consultant, Proctor, Research Support. NuMED: Consultant. AVANIA, LLC: CEC Chair. Xeltes: DSMB.

Ethical Approval: Not applicable.

Consent for Publication: Not applicable.

\section{Accurate Prediction of Systolic Peak-to-Peak Gradient in Children with Pulmonary Valve Stenosis}

\section{Salvador Rodriguez Franco $^{1,2}$, Jenny Zablah ${ }^{1,2}$, Benjamin Frank $^{1}$, Gareth Morgan ${ }^{1,2,3}$}

'The Heart Institute, Children's Hospital Colorado, Aurora, USA.

${ }^{2}$ University of Colorado, School of Medicine, Aurora, USA.

${ }^{3}$ Department of Cardiology, University of Colorado Hospital, Aurora, USA

To evaluate the correlation between transthoracic echocardiography (TTE) derived gradients and invasive RV-PA peak-to-peak systolic gradient (iPG) and the predictability of these values in children with pulmonary valve stenosis (PVS). Historically, correlations have been found between the Doppler-derived peak echo gradient (ePG), using the modified Bernoulli equation and invasive hemodynamic indices. This has led to the ePG being a widely used estimate of the degree of stenosis and an indicator of timing to consider intervention in children with PVS. However, large disparities observed between these invasive and non-invasive measurements have called into question the reliability of $\mathrm{ePG}$ to screen patients for consideration of pulmonary valvoplasty. We performed a retrospective chart review of 119 children who underwent pulmonary valvoplasty between January 2010 and January 2020 and selected 51 patients who had "simple" PVS in whom a TTE was performed within 6 months prior to the procedure. We evaluated the correlation between TTE parameters [Maximum velocity (Vmax), Mean Gradient (MG), ePG], and iPG. We then used the mathematical functions resulting from the correlation models to predict iPG. All the assessed models were statistically significant and presented a strong correlation. When the differences between echocardiographic parameters and iPG were evaluated, ePG demonstrated the least accuracy in predicting iPG, erroneously estimating the real value by $>10 \mathrm{mmHg}$ in $66 \%$ of cases. The MG presented a more accurate estimation of $\mathrm{PG}$, showing a narrower range of variance, with an error of $>10 \mathrm{mmHg}$ in less than $30 \%$ of cases. We then modeled several mathematical functions of $\mathrm{Vmax}, \mathrm{MG}$, and $\mathrm{ePG}$ to predict iPG, and found that a derived function of Vmax (fiPG) distributed by BSA ( $\mathrm{iPG}=12.7[\mathrm{Vmax}]-17.3[\mathrm{BSA}]$ ) produced the most accurate results. There is a strong correlation between pre-procedural TTE parameters and iPG, but when ePG is used to estimate iPG, up to two-thirds of cases could be inappropriately characterized. We found that MG was a better predictor and that a mathematical function derived from Vmax and BSA (fiPG) was the most accurate predictor 
of iPG. Key words: Congenital Heart defects, catheter peak gradient, doppler peak gradient, mathematical modeling.

\section{ETHICS DECLARATIONS}

Conflict of interest: The authors declare that they do not have any conflict of interests.

Ethical Approval: Data collection and analysis were conducted under the permission of the Colorado Multi Institutional Review Board (IRB19-2292).

Consent for Publication: Not applicable.

\section{The Evolution of Tricuspid Regurgitation After Percutaneous Patent Ductus Arteriosus Closure in Ex- preterm Infants Under 2 Kilograms}

\author{
Salvador Rodriguez Franco $^{1,2}$, Barry O'Callaghan ${ }^{1,2}$, Jenny \\ Zablah $^{1,2}$, Gareth Morgan ${ }^{1,2,3}$ \\ ${ }^{1}$ The Heart Institute, Children's Hospital Colorado, Aurora, USA. \\ ${ }^{2}$ University of Colorado, School of Medicine, Aurora, USA. \\ ${ }^{3}$ Department of Cardiology, University of Colorado Hospital, Aurora, \\ USA
}

To evaluate incidence and evolution of new tricuspid regurgitation (TR) after percutaneous patent ductus arteriosus (PDA) closure in preterm infants under $2 \mathrm{~kg}$. Multiple advances in techniques and technology in cardiac catheterization have facilitated percutaneous closure of neonatal PDAs. A higher rate of complications has been suspected in this setting, but robust scientific evidence concerning this association is not yet available. TR has been a recognized significant complication, gaining notoriety with the use of the Piccolo device over the last few years. We performed a single-center retrospective cohort study. We included 29 patients under $2 \mathrm{~kg}$ who underwent percutaneous PDA closure between January 2019 and December 2020. This cohort was then matched with a control group of sub $2 \mathrm{~kg}$ patients who did not have percutaneous PDA closure at a ratio of $2: 1$ $(\mathrm{n}=58)$. Echocardiographic data points were collected prior to catheterization and at intervals up to 1-year post-procedure. To standardize the temporal change in TR, we measured the zone of convergence and normalized it with the body surface area related to time. TR after percutaneous PDA closure was seen in 9 cases $(31.1 \%)$, of which 8 cases were classified as mild-to-moderate $(27.6 \%)$ and 1 case as severe (3.4\%). At the end of follow-up, TR persisted in $5 / 9$ cases $(17.2 \%)$; however, no increases in TR, clinical complications, or need for reintervention were noted for these patients. In the control group of 58, we incidentally noted two patient with moderate to severe TR which diminished over time. Compared to the control group, our cohort did not present significant difference in terms of survival or weight gain characteristics. TR remains a worrying likely mechanical consequence of catheter and device manipulation in percutaneous preemie duct closure. We should strive to decrease such complications by technical and procedural improvement. Despite this, no patient in our cohort has required specific medical or surgical reintervention for TR, and the echocardiographic trend is tending towards a decreasing grade of TR over time.

Keywords Tricuspid regurgitation, PDA closure, preterm, Sub 2 kg.

\section{ETHICS DECLARATIONS}

Conflict of interest: The authors declare that they do not have any conflict of interests.
Ethical Approval: Data collection and analysis were conducted under the permission of the Colorado Multi Institutional Review Board (IRB 21-3673).

Consent for Publication: Not applicable.

\section{Frame Fracture Frequency and Heatmap Distribution During Follow-up of the GORE Cardioform ASD Device}

\author{
Gareth Morgan $^{1,2,3}$, Salvador Rodriguez Franco ${ }^{1,2}$, Ryan \\ Leahy $^{1,2}$, Jess Randall ${ }^{1}$, Jenny Zablah ${ }^{1,2}$ \\ ${ }^{1}$ The Heart Institute, Children's Hospital Colorado, Aurora, USA. \\ ${ }^{2}$ University of Colorado, School of Medicine, Aurora, USA. \\ ${ }^{3}$ Department of Cardiology, University of Colorado Hospital, Aurora, \\ USA
}

To evaluate the frequency and characteristics of Gore Cardioform ASD Device Frame Fractures (FF) and explore potential associations in patient and procedural practice variables. The latest device approved by the FDA for atrial septal defect (ASD) closure is the GORE ${ }^{\circledR}$ Cardioform ASD occluder (GCA). Despite a very low incidence of complications having a direct impact on patient wellbeing, a significant amount of FFs have been observed in the GCA at short to medium-term follow-up, being described in almost one-third of the devices implanted during clinical trials. We performed a retrospective single-center chart review of 54 patients who received a GCA between January 2017 and December 2019, and selected 35 patients in whom a 6-month fluoroscopic follow-up was performed. We evaluated the number and type of fractures based on this fluoroscopic assessment and devised a simple nomenclature system based on three-dimensional (3D) reconstruction of the device's fluoroscopic images. We also evaluated the association between the position and number of fractures, and patient and procedural characteristics. The FF incidence in our population was $42.9 \%$ at a 6-month follow-up. The factors statistically associated with FF were the size of the defect and the size of the device implanted. FFs were most frequently located in a central position in the right atrial disc. By $3 \mathrm{D}$ evaluation, the most common anatomical position for fractures was the anteroinferior quadrant of the right atrial disc. At the time of manuscript submission, no echocardiographic or clinical sequelae were noted in our population. The incidence of frame fractures in our population was higher than that observed in published literature, albeit using more intense image scrutiny than that required by other cited studies. We noted that the central portion of the anteroinferior quadrant of the right atrial disc was the most susceptible to FF and found that the defect size and the device size were the only statistically associated factors.

\section{ETHICS DECLARATIONS}

Conflict of interest: The authors declare that they do not have any conflict of interests.

Ethical Approval: Data collection and analysis were conducted under the permission of the Colorado Multi Institutional Review Board (IRB 17-0059).

Consent for Publication: Not applicable. 


\section{Does the Benefit of Transcatheter PDA Closure Outweigh the Harm of Contrast Nephropathy in Infants weighing Under $2 \mathrm{~kg}$ ?}

\section{Christopher Herron $^{1}$, Thomas Forbes ${ }^{1,2}$, Daisuke Kobayashi ${ }^{1,2}$ \\ ${ }^{1}$ Children's Hospital of Michigan, Detroit, USA. ${ }^{2}$ Central Michigan University College of Medicine, Mount Pleasant, USA}

Angiography using contrast provides an accurate measurement of a patent ductus arteriosus (PDA) and facilitates the effective and safe placement of a PDA device. Contrast nephropathy is a concern in clinically ill infants, especially with the presence of renal insufficiency. We hypothesized that the benefit of transcatheter PDA closure outweighs the harm of contrast nephropathy in extremely premature infants.To evaluate the short-term effect of contrast use on serum creatinine in infants weighing under $2 \mathrm{~kg}$ who underwent transcatheter PDA closure. This is a single centered retrospective study. The study cohort was infants (aged $\geq 10$ days) weighing under $2 \mathrm{~kg}$ who underwent successful transcatheter PDA closure using angiography and had follow-up data of serum creatinine level at least $24 \mathrm{~h}$ post-device. Pre-existing renal insufficiency was defined as having serum creatinine $\geq 1.0 \mathrm{mg} / \mathrm{dl}$. The data were compared by a paired t-test. A total of 48 infants (gestational age $25 \pm 2$ weeks) underwent PDA closure using an Amplatzer Piccolo PDA occluder at the age of $32 \pm 16$ days. The weight at the time of catheterization was $930 \pm 340 \mathrm{gm}$ with the contrast amount being $2.3 \pm 1.1 \mathrm{ml} / \mathrm{kg}$. Overall serum creatinine level was $0.66 \pm 0.52 \mathrm{mg} / \mathrm{dl}$ at baseline and $0.60 \pm 0.46 \mathrm{mg} / \mathrm{dl}$ at $24 \mathrm{~h}$ post-device $(\mathrm{p}=0.051$, Figure $)$. In 7 infants with pre-existing renal insufficiency (serum creatinine $1.57 \pm 0.7 \mathrm{mg} / \mathrm{dl}$ ), serum creatine level significantly improved from $1.38 \pm 0.69 \mathrm{mg} / \mathrm{dl}$ at $24 \mathrm{~h}$ to $0.88 \pm 0.49 \mathrm{mg} / \mathrm{dl}$ at 1 week post-device $(p=0.015)$. Despite the use of contrast, serum creatinine levels significantly improved with PDA closure even in infants with preexisting renal insufficiency. Improving renal perfusion with PDA closure does outweigh the harm of contrast nephropathy. Angiography should not be discouraged because of the concern for contrast nephropathy.

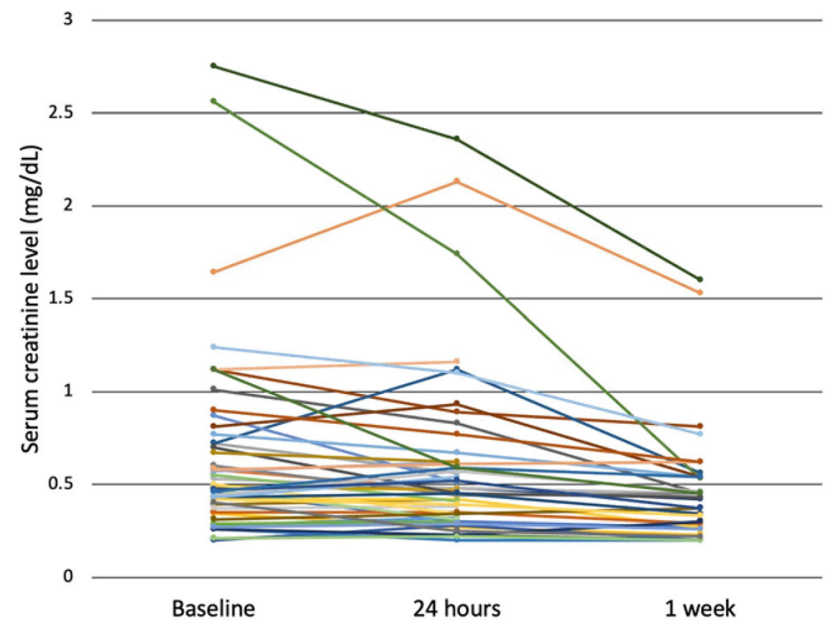

ETHICS DECLARATIONS

Conflict of interest: The authors declare that they do not have any conflict of interests.

Ethical Approval: This research was approved by the IRB at Central Michigan University and the Detroit Medical Center.

Consent for Publication: Not applicable.
032. Enalapril Improves Pulmonary Artery Pressure for Infants with Single Systemic Right Ventricle: Analysis of the Pediatric Heart Network Infant Single Ventricle Public Use Dataset

\section{Danielle Strah, Kevin Weinberger, Katie Kowalek, Andrew Hoyer, Michael Seckeler}

University of Arizona, Tucson, USA

\begin{tabular}{|c|c|c|c|}
\hline Factor & $\begin{array}{c}\text { Enalapril } \\
(n=61)\end{array}$ & $\begin{array}{l}\text { Placebo } \\
(\mathrm{n}=70)\end{array}$ & $p$ \\
\hline \begin{tabular}{|l} 
Ventricular end-diastolic pressure $(\mathrm{mmHg})$ \\
\end{tabular} & $8.1 \pm 4.6$ & $8.1 \pm 3.1$ & 0.932 \\
\hline Aortic systolic pressure (mmHg) & $81.3 \pm 15.3$ & $84.7 \pm 14.9$ & 0.213 \\
\hline Aortic diastolic pressure (mmHg) & $36.7 \pm 7.3$ & $37.6 \pm 7.6$ & 0.517 \\
\hline Aortic mean pressure (mmHg) & $54.1 \pm 9.9$ & $56.1 \pm 9.9$ & 0.250 \\
\hline Pulmonary artery mean pressure $(\mathrm{mmHg})$ & $12(11.8,14.3)$ & $15(12,16.8)$ & 0.020 \\
\hline \begin{tabular}{|l} 
Pulmonary artery mean wedge pressure $(\mathrm{mmHg})$ \\
)
\end{tabular} & $10(8,13)$ & $11(7.3,14)$ & 0.930 \\
\hline Systemic atrial mean pressure $(\mathrm{mmHg})$ & $6.5 \pm 2.3$ & $6.6 \pm 2.1$ & 0.678 \\
\hline Qp:Qs & $1(0.8,1.3)$ & $1(0.7,1.3)$ & 0.888 \\
\hline Qp:Qs $\leq 1.0$ & $31(51 \%)$ & $36(51 \%)$ & 0.945 \\
\hline Transpulmonary gradient $(\mathrm{mmHg})$ & $6(5,8)$ & $6(5,9)$ & 0.780 \\
\hline Pulmonary resistance (indexed Wood units) & $1.81(1.38,2.47)$ & $1.85(1.28,2.78)$ & 0.876 \\
\hline
\end{tabular}

To analyze the Pediatric Heart Network Infant Single Ventricle (PHN-ISV) public use dataset to determine if enalapril improved multiple hemodynamic measures, including ventricular end-diastolic pressure, pulmonary artery pressure and pulmonary vascular resistance in infants with a single systemic right ventricle. Infants with single systemic right ventricle are at increased risk for diastolic dysfunction compared to those with single systemic left ventricle. Because of this, they may benefit from systemic afterload reduction or the anti-fibrotic effects from ACE-inhibitors. There are also data to suggest an independent decrease in pulmonary vascular resistance with the use of ACE-inhibitors, which could also benefit these patients. In the PHN-ISV trial, 230 infants were randomized to enalapril or placebo and followed for 14 months. Clinical, echocardiographic and invasive pre-Stage II hemodynamic data were collected. While multiple hemodynamic parameters were analyzed in the original trial, infants with single systemic right ventricle were not analyzed separately and pulmonary vascular resistance was not assessed. Retrospective review of the PHN-ISV public use dataset for patients with single systemic right ventricle. Demographic and preStage II hemodynamic data for those who received enalapril and those who received placebo were compared, including pulmonary artery pressure and pulmonary flow, measured by the Fick equation. To calculate pulmonary resistance, the hemoglobin level reported closest to the time of the catheterization was utilized and the oxygen consumption was estimated using the recently published equation by Seckeler and colleagues. Data were compared using independent t-test for normally distributed data, Mann-Whitney $U$ test for nonnormally distributed data and $\chi^{2}$ for categorical data. A $p$-value $<$ 0.05 was considered statistically significant. 131 patients were identified, 61 treated with enalapril and 70 given placebo. Demographics and hemodynamic data are shown in the Table. There were no differences in ventricular end-diastolic pressure or pulmonary resistance, but the enalapril group had significantly lower mean pulmonary artery pressure (12 vs. $15 \mathrm{mmHg}, p=0.020)$. 
Using data from the PHN-ISV trial, we found that, while there were no improvements in ventricular end-diastolic pressure or pulmonary resistance for infants with single systemic right ventricle who received enalapril, there was a significant improvement in pulmonary artery pressure which could have longer term benefits through staged surgical palliation.

\section{ETHICS DECLARATIONS}

Conflict of interest: The authors declare that they do not have any conflict of interests.

Ethical Approval: Not applicable.

Consent for Publication: Not applicable.

\section{Novel Use of Double Kissing Crush Technique to Stent Complex Pulmonary Artery Stenosis in a Child with Alagille Syndrome}

\section{Danielle Strah, Kwan Lee, Michael Seckeler}

\section{University of Arizona, Tucson, USA}

We present a 12-year-old female with Alagille syndrome with ventricular septal defect (VSD) and complex bilateral pulmonary artery stenoses status post several catheterizations for pulmonary artery rehabilitation to try to improve her marked right ventricle hypertension. She had a complex multivessel pulmonary artery stenosis which was successfully treated using the double kissing (DK) crush technique for stent placement. The procedure was performed under general anesthesia. A long sheath was positioned in the proximal right pulmonary artery. Angiography identified a complex trifurcation stenosis in the right lower lobe artery with an angiographically similar appearance to a complex coronary artery bifurcation lesion (a). As she had had inadequate response to angioplasty alone in prior catheterizations, it was felt that stent placement would be required. In collaboration with the coronary interventional team, it was decided to perform DK crush bifurcation stenting. Coronary wires were positioned into both branches and kissing pre-dilatation was performed with two $2.5 \times 12 \mathrm{~mm}$ balloons. A Resolute Onyx $4.0 \times 15 \mathrm{~mm}$ DES was positioned in the smaller branch with slight protrusion, with a $5.0 \times 12 \mathrm{~mm}$ NC Euphora in the larger branch. The stent was deployed, after which the balloon was removed, and the $5.0 \mathrm{~mm}$ balloon was used to crush the protruding segment of the deployed stent. The stent was then re-wired with a third wire and the first wire was removed. Kissing balloon angioplasty was performed with two $4.0 \times 12 \mathrm{~mm}$ balloons. A $5.0 \times 18 \mathrm{~mm}$ Resolute Onyx DES was deployed in the larger ramus over the ostium of the recently stented branch (b, c). Proximal optimization was performed with a $6.0 \times 8 \mathrm{~mm}$ NC Emerge balloon, after which the branch was rewired. Final kissing balloon angioplasty was performed with a 4.0 and a $5.0 \mathrm{~mm} \mathrm{NC}$ balloons (d). There was good distal flow beyond the stents, including the third jailed branch (e). Qp:Qs increased from 1.4:1 at baseline to 2.1:1 after intervention making her a potential candidate for VSD closure. Our patient's case appears to be the only case we know of that has utilized the DK crush technique in pulmonary arteries, which may prove to be an important treatment method for patients with complex pulmonary artery disease. As the complexity of patients increases, collaboration between congenital and structural interventionalist becomes vital to optimize outcomes.

ETHICS DECLARATIONS

Conflict of interest: The authors declare that they do not have any conflict of interests.

Ethical Approval: Not applicable.

Consent for Publication: Not applicable.
034. Novel Technique for Transcatheter closure of Sinus Venosus Atrial Septal Defect: The Temporary Suture-Holding Technique [Boudjemline Technique]

\author{
Yahia Hejazi, Ziyad Hijazi, Hesham Al-Saloos, Younes \\ Boudjemline
}

Sidra Medicine, Doha, Qatar.

Surgical correction of Sinus Venosus Atrial Septal Defect (SVASD) is considered the standard of care. However, transcatheter closure has been recently reported with good short and mid-term results. Most publications reported the implantation of covered stent in superior vena cava (SVC) in order to cover the ASD and simultaneously redirect right upper pulmonary vein (RUPV) flow to left atrium. However, positioning and stabilization of this stent remain challenging. We describe here a "simple" technique that optimizes covered stent position. We reviewed the patients with SVASD who underwent transcatheter closure using the suture technique. The conventional pre-closure catheterization assessment included the following steps: (1) full hemodynamic and angiographic data; (2) creation of venovenous circuit connecting right femoral vein (RFV) with right jugular vein (RJV) and (3) calibration of the SVC with balloon sizing and venogram of RUPV to ensure patency with balloon fully inflated. The stent placement and positioning differ from the conventional techniques. The stent was prepared ex vivo as followed: a suture (MERSILK Ethicon KS-75) was inserted through one of the distal stent struts not holding the PTFE. It was, then pulled in a way to have two equal ends securing the stent. A Mustang $12 \times 6 \mathrm{~cm}$ balloon was then inserted from the RFV over the wire rail and exteriorized through the SVC sheath. The chosen covered stent was crimped over the balloon or on the shaft taking care to position the suture at the SVC side of the stent. The stent-balloon assembly was then pulled from the RFV into the SVC sheath. After positioning the stent, the balloon was expanded. At this stage, the stent was not opposed to the wall and was held by the suture. The balloon was then removed and a larger balloon was placed. Optimal positioning was made under echocardiographic and fluoroscopic guidance. Flaring of the right atrial end of the stent with balloon to close the atrial shunt was made initially and the stent was then stabilized at the SVC level. If an additional stent was required, it was placed before removing the suture. The suture was then pulled at the end of the procedure when placement and stability were confirmed. To avoid pulling up the stent while removing the suture, a balloon was inflated in the stent. We report our acute and mid-term follow-up data. Six patients underwent transcatheter closure of SVASD at our center using the suture technique. No embolization was noted during the procedure and at followup. No patient required additional stent placement at SVC level. Residual tiny ASD shunt was observed in three cases. One of them had an additional covered stent placed to cover the residual shunt. This patient had a small intra-stent clot which was not seen after 2 days of heparin infusion. The other two residual shunts were negligible and disappeared during follow-up (Qp:Qs were close to 1:1). One patient had insignificant pericardial effusion. Significant reduction in right heart size was demonstrated during follow-up. Cardiac CT angiography was performed in $5 / 6$ patients and in all confirmed patency of stents and flow of the re-directed pulmonary veins. Our report presents a novel suture technique that provides additional safety profile to the transcatheter correction of SVASD avoiding secondary embolization.

\section{ETHICS DECLARATIONS}

Conflict of interest: The authors declare that they do not have any conflict of interests.

Ethical Approval: Not applicable.

Consent for Publication: Not applicable. 
035. Transcatheter Pulmonary Valve Placement Within Existing Stenotic and Insufficient Right Ventricle to Pulmonary Artery Conduit/Bioprosthetic Pulmonary Valves in Patients with One and a Half Ventricle Repair is a Viable Alternative to Surgical Replacement of the Conduit

\author{
Sharib Gaffar ${ }^{1}$, Gira Morchi ${ }^{2}$, Sanjay Sinha ${ }^{1,2}$, Michael Recto ${ }^{2}$ \\ ${ }^{1}$ UCLA Mattel Children's Hospital, Los Angeles, USA. ${ }^{2} \mathrm{CHOC}$ \\ Children's Hospital of Orange County, Orange, USA
}

Transcatheter pulmonary valve (TPV) implantation is accepted therapy for patients who have previously undergone placement of a prosthetic right ventricular outflow tract (RVOT) conduit in the setting of conduit stenosis and insufficiency. The purpose of this abstract is to report our experiences of TPV placement in 3 patients with pulmonary atresia with intact ventricular septum who had previously undergone the one and a half ventricle type of repair consisting of a bidirectional Glenn shunt and surgically placed right ventricle to pulmonary artery (RV-PA) conduit, and later developed conduit stenosis and insufficiency. Three patients ( 2 females, 1 male) with mean age 15.3 years (range 13-17 years), and mean weight $72 \mathrm{~kg}$ (range $54-88 \mathrm{~kg}$ ) developed severe pulmonary valve insufficiency and mild-moderate conduit/pulmonary valve stenosis. All patients had initially undergone placement of a central shunt in infancy followed by a bidirectional Glenn and RV-PA conduit (Contegra conduit in 1 patient, bioprosthetic pulmonary valve in 2 patients). One patient with significant conduit stenosis (Contegra Conduit, Medtronic, MN) required balloon angioplasty and stent implantation (Palmaz XL 3110 stent, Cordis, CA) prior to placement of a $20 \mathrm{~mm}$ Melody valve (Medtronic, MN). One patient required splitting of the existing $19 \mathrm{~mm}$ bioprosthetic pulmonary valve with a $20 \mathrm{~mm}$ Atlas balloon (Bard, AZ) to relieve conduit stenosis prior to placement of a $22 \mathrm{~mm}$ Melody valve. One patient with mild stenosis and severe bioprosthetic pulmonary valve insufficiency underwent placement of a $20 \mathrm{~mm}$ Melody valve with resolution of bioprosthetic pulmonary valve stenosis and insufficiency. All three patients had successful implantation of TPV (Melody valve) with resolution of conduit/bioprosthetic pulmonary valve stenosis and insufficiency. There were no complications. Transcatheter pulmonary valve implantation within an existing stenotic and insufficient RV-PA conduit in patients with one and a half ventricle circulation relieves RV-PA conduit stenosis and insufficiency, and is a viable alternative to surgical replacement of the existing conduit.

ETHICS DECLARATIONS

Conflict of interest: The authors declare that they do not have any conflict of interests.

Ethical Approval: Not applicable.

Consent for Publication: Not applicable.

\section{Left Ductal Stent to Disconnected LPA, RVOT Stent and Right Duct Closure in the Context of Severe Form of Tetralogy of Fallot's, Double Aortic Arch and Disconnected LPA}

\section{Ahmed Adel Hassan, Mahmoud Alsoufi, Mohamed Kasem}

Al Jalila Childrens, Dubai, UAE

We are presenting a case of complex congenital heart disease presenting with duct dependent pulmonary circulation with low birth weight that was palliated in the cath lab in our hospital. Initial echo showed Tetralogy of Fallot with narrow LPA origin, PDA supply the LPA, Muscular VSD. Cardiac CT showed Double aortic arch with dominant left arch, LSCA and LCA arise from the left arch, and RSCA and RCA arise from the right arch. The main Pulmonary artery is small connects only to RPA. Right duct arise from the RCA and connect to RPA. Vertical Left duct arise from the left arch and connect to the LPA. Second duct from the right arch which connect to the right pulmonary artery. There is discontinuity between the LPA and MPA. We took the patient to the cath lab at age of nine days when she was $2.9 \mathrm{KG}$. The rationale behind the procedure was the low saturation, disconnected LPA. we stented the Left PDA to keep flow to LPA using 3/15 mm DES, but the diastolic pressure was low down to $19 \mathrm{mmHg}$, no ECG changes, but saturation still was below $85 \%$ in air. We decided to stent the RVOT to get enough $\mathrm{O} 2$ saturation for the next few months, this was achieved with 5/16 DES, and saturation went up to $90 \%$. We closed the Right PDA to reduce the diastolic steal and improve the diastolic blood pressure. This was achieved by using MVP 7Q plug via right axillary artery access. The Diastolic pressure came up from 19 to $25 \mathrm{mmHg}$. The baby was extubated the following day, and discharged after few days on dual anti-platelets' therapy. Patient was then observed for 2 weeks in the NICU and was doing well so discharged and are following in our out-patient cardiology department.

\section{ETHICS DECLARATIONS}

Conflict of interest: The authors declare that they do not have any conflict of interests.

Ethical Approval: Not applicable.

Consent for Publication: Not applicable.

037. Simulated Transcatheter Intervention on 3DPrinted Prototypes as Multimodal Strategic Planning for Complex Structural Heart Disease Repair: A Novel and Emerging Joint Program in a Biomedical Engineering Research Center to Support Cardiovascular Surgery Programs That Improve The Strategic Planning of Endovascular Repair of Complex Congenital Heart Disease in a Development Country

Manuel Ricardo Tellez Alvarez, Martha Lucia Velasco, Carlos Latorre, Maria Lucia Arango

Fundacion Clinica Shaio, Bogota, Colombia

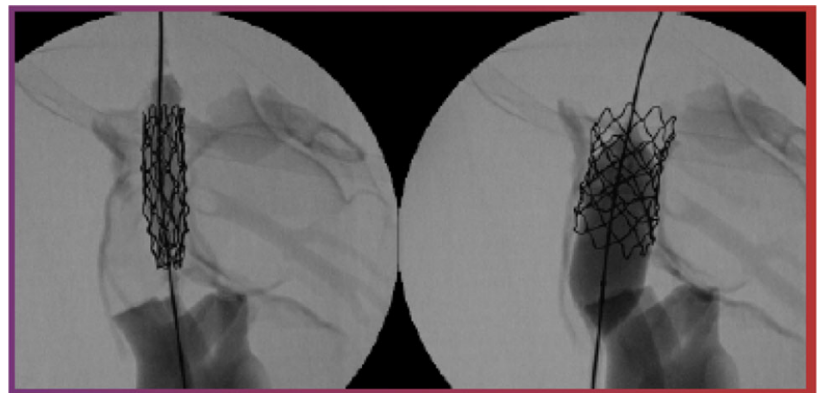



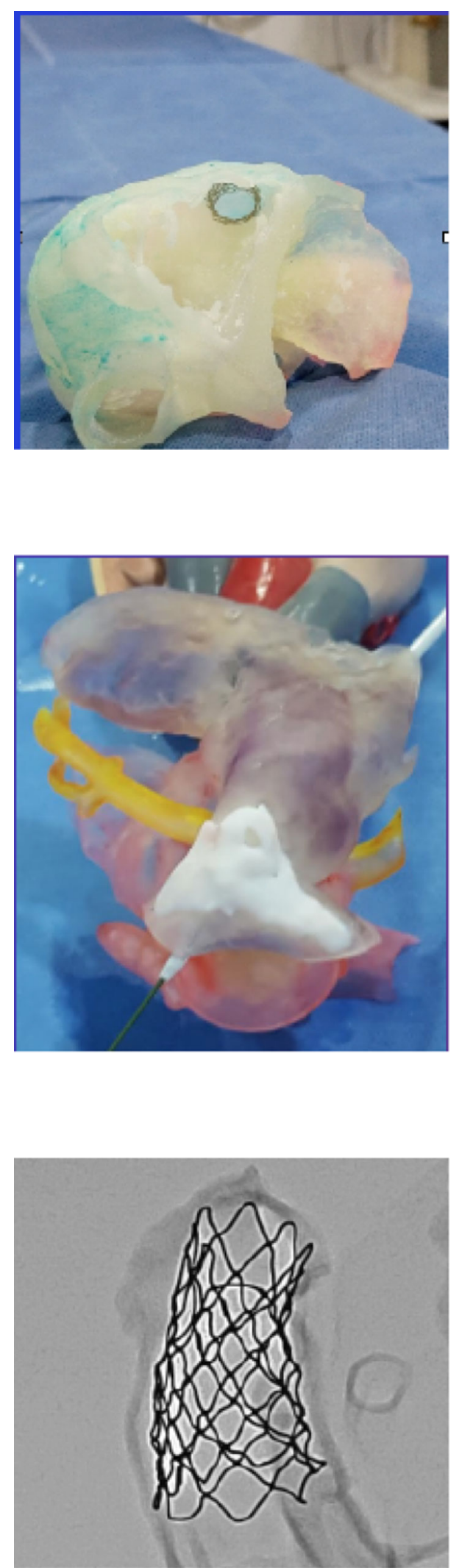

Structural heart disease can be primary congenital lesions, or acquired as part of late post-surgical or degenerative sequelae. In pediatric and early adulthood, the highest incidence is found by pathologies that compromise the flow of the pulmonary circuit and implicitly bring RVOT dysfunction.The percutaneous implantation of biological valves is increasingly used in patients with primary RVOT or postsurgical residual dysfunction due to the use of prosthetic material. There are a large number of anatomical variations that lead to the appearance of these obstructive or incompetent lesions. A correct selection of the size of the new prosthesis is required for its subsequent approximation, positioning, anchorage and impaction, the anatomical precision of the RVOT is essential. 3D model printing is a prototyping technology that provides the possibility of producing tangible replicas of the geometry of a specific organ, reconstructed from three-dimensional diagnostic images. Hands on Training, also known as experiential learning, is in other words the act of learning by doing and reproducing. Among the multiple applications of 3D printing in cardiology, are the positioning tests of devices prior to the procedure. It is intended to make a comparative account and analysis with the world literature of the first experiences in Colombia of the use of this sequential strategy. A joint program was started in a biomedical engineering research center to support 4 cardiovascular surgery programs to improve the strategic planning of endovascular repair of complex congenital heart disease. We present our first three cases of patients with a history of complex trunk heart disease with multiple surgical corrections and late RVOT dysfunction. Considering the complexity of a new surgical approach and the difficulty in its post-surgical anatomy, it was decided to acquire digital CT angio images with volumetric reconstruction and digital impression of a 3-dimensional prototype and carry out a simulation under fluoroscopy and cineangiography, defining the minimum details of the anchorage zone and the selection of the diameter of the materials and supplies as well as their sequence of approach and subsequent impact on the prototype. The patients were finally taken to the planned procedures with successful results. The information obtained by printing prototypes and models acquired from volumetric CT images was definitive in the strategic planning of the approach and sequencing of the procedure as well as in the selection of the material used. The use of 3D printed models resulted in a more precise selection of materials and operational strategies. By using the technological simulation strategy, it is possible to foresee the therapeutic approach sequence and to avoid and correct probable errors. The development of a prototyping program with $3 \mathrm{D}$ models has the potential to improve the results of endovascular and complementary surgical repair in complex CHD.

ETHICS DECLARATIONS

Conflict of interest: The authors declare that they do not have any conflict of interests.

Ethical Approval: Not applicable.

Consent for Publication: Not applicable.

\section{Transcatheter Edge-to-Edge Repair of the Systemic Atrioventricular Valve in Adults with Congenital Heart Disease}

Laith Alshawabkeh, Ehtisham Mahmud, Howaida El-Said, Ryan Justin, Erik Echegaray, Megan Kraushaar, Maria Moceri-Casas, Ryan Reeves

\section{University of California San Diego, La Jolla, USA}

Transcatheter edge-to-edge repair (TEER) using the MitraClip system is approved to treat primary or secondary (functional) mitral regurgitation. Currently, surgical intervention is the only available option for native systemic atrioventricular valve regurgitation (AVV/AVVR) among adults with congenital heart disease (CHD). TEER has been 
reported in a handful of cases of adults with CHD. We describe the most extensive single-center experience using this technology in adults with CHD. Between July 2019 and April 2021, five patients with complex CHD and severe, symptomatic AVVR underwent TEER using the MitraClip system. All patients were deemed at high surgical risk by a CHD surgeon, and all provided informed consent for the compassionate use of the device. Median age was 39 years (IQR 35.5 to 60.6 ), and two were female. Three patients with [S,L,L] transposition of the great arteries and a systemic right ventricle (one with additional history of ventricular septal defect repaired in childhood) and developed severe functional tricuspid regurgitation. One patient with $[S, L, D]$ dextrocardia, double outlet right ventricle, mildly hypoplastic tricuspid valve, and secundum atrial septal defect palliated at age 35 with a bidirectional Glenn anastomosis and later developed severe, symptomatic primary mitral regurgitation due to a cleft in the anterior leaflet. The last patient was born with [A,D,D] Heterotaxy syndrome, unbalanced right-ventricle dominant complete atrioventricular canal defect, and total anomalous pulmonary venous connection palliated to an extracardiac Fontan circulation, and later developed torrential both primary and functional AVVR and cardiogenic shock. TEER was performed to stabilize the patient as a bridge to transplantation. Significant pre-procedural planning via 3-dimensional reconstruction was required to guide the procedure. The challenge with trans-septal access (or trans-baffle in the patient's case with Fontan circulation) was to align the device with the AVV leaflets properly. Three patients required two clips, and two patients required one clip. One patient had a single-leaflet detachment of one of the clip arms with no change in regurgitation severity. The median procedure time was 242 min (IQR 155 to 337). A standard six-week follow-up echocardiogram showed stable AVVR and unchanged systemic ventricular function. After a median follow-up of 236 days (IQR 28 to 493), AVVR remained stable. One patient died from a drug overdose, and one underwent heart transplantation. NYHA functional class improved in all except one patient who had functional class 1 , to begin with, but reported symptomatic improvement following the intervention. TEER in adults with CHD is feasible, results in a reduction in AVVR and improvement in NYHA functional class. Considerable pre-procedural planning is necessary, and combined expertise from adult CHD, CHD imaging, structural and CHD interventional cardiologists.

ETHICS DECLARATIONS

Conflict of interest: The authors declare that they do not have any conflict of interests.

Ethical Approval: Not applicable.

Consent for Publication: Not applicable.

\section{Use of the PK Papyrus Covered Coronary Stent in the Treatment of Kawasaki Disease-Associated Giant Coronary Artery Aneurysms}

\author{
$\underline{\text { Iman Naimi }}^{1}$, Brian Morray ${ }^{1}$, Zachary Steinberg ${ }^{2}$ \\ ${ }^{1}$ Seattle Children's Hospital, University of Washington School \\ of Medicine, Seattle, USA. ${ }^{2}$ University of Washington Medical \\ Center, University of Washington School of Medicine, Seattle, USA
}

Kawasaki disease (KD) is an acute vasculitis that can cause coronary artery (CA) inflammation and aneurysm formation leading to early obstructive disease. The success of percutaneous interventions in this condition has been limited. The PK Papyrus stent (Biotronic, Inc. Berlin, Germany) is a low profile, polyurethane covered, cobalt chromium stent approved by the Food and Drug Administration for the treatment of CA perforation under a Humanitarian Device Exemption. Use of this stent in the treatment of stenotic giant coronary aneurysms (GCA) has not been described. This case series describes the use of the PK Papyrus stent to simultaneously revascularize and exclude stenotic GCA in young patients with KD. This is a single center, retrospective study of three patients with GCA secondary to remote $\mathrm{KD}$ who underwent transcatheter implantation of the PK Papyrus covered stent. Institutional Review Board (IRB) permission for stent use was granted in each case through either emergency or compassionate use applications. Three patients $(9,14$ and 17 years old) underwent successful stent implantation. Patient 1 had previously undergone left internal mammary artery (LIMA) to mid-left anterior descending (LAD) bypass for complete occlusion of the proximal LAD and underwent successful placement of a $3.5 \mathrm{~mm} \times 15 \mathrm{~mm}$ Papyrus stent from the left main to left circumflex (LCX) to exclude a partially thrombosed saccular aneurysm arising off the communicating ostium of the occluded LAD. Patient 2 underwent revascularization of a highly calcified and stenotic right coronary artery (RCA) GCA with rotational atherectomy and Shockwave lithotripsy followed by deployment of a $3.5 \mathrm{~mm} \times 26$ $\mathrm{mm}$ Papyrus stent. Patient 3 underwent placement of $3.5 \mathrm{~mm} \times 15$ mm Papyrus stent to cover a stenosed RCA aneurysm in the setting of an inferior wall myocardial infarction. Immediate post-procedural angiography demonstrated relief of stenoses and complete GCA occlusion in all cases. There were no procedural deaths. All three patients have remained asymptomatic at one year follow-up. Patients 1 and 2 underwent follow-up surveillance angiography at 10- and 11-months post procedure, respectively. Patient 1 was found to have modest, non-obstructive in-stent restenosis (ISR). Patient 2 was found to have significant ISR and underwent placement of a $3.5 \mathrm{~mm} \times 34$ mm Resolute Onyx Drug Eluting Stent (Medtronic, Minneapolis, MN, USA). Patient 3 will undergo surveillance angiography in June 2021. Percutaneous coronary intervention for the treatment of GCA remains complicated by early restenosis. There are limited data on outcomes of patients who undergo GCA exclusion with covered stents. In this series, all patients underwent successful relief of aneurysm-associated stenosis and exclusion of the aneurysms and have not experienced any complications with short-term follow-up. Two of the three patients have undergone follow-up angiography demonstrating persistent exclusion of the aneurysm from the circulation and varying degrees of ISR. The PK Papyrus stent is not coated with antiproliferative medications. Our findings suggest that KD patients who undergo GCA exclusion with the PK Papyrus stent may benefit from either concomitant drug eluting stent implantation within the covered stent or should be followed closely with a low threshold for additional drug-eluting stent implantation as necessary. The need for localized drug delivery to limit ISR and the impact of covered stent placement on long-term anticoagulation needs are important topics that will require more study. While longer term data are lacking, use of the PK Papyrus covered stent may be a valuable tool in dealing with stenotic and thrombogenic aneurysms in KD patients.

ETHICS DECLARATIONS

Conflict of interest: The authors declare that they do not have any conflict of interests.

Ethical Approval: Not applicable.

Consent for Publication: Not applicable. 


\section{Incidence of Infective Endocarditis in Patients Undergoing Surgical or Transcatheter Pulmonary Valve Replacement: A Single-Center Experience}

\section{Ashish Saini, Rodrigo Cavalcante, Lourdes Prieto}

\section{Nicklaus Children's Hospital, Miami, USA}

Infective endocarditis (IE), with an incidence of about $0.05-0.12$ per 1000 patient-years, is associated with significant morbidity and mortality in patients with congenital heart disease. Surgically implanted Contegra conduit and the Melody transcatheter pulmonary valve, derived from bovine jugular vein, have been reported to have an increased incidence of IE. The purpose of this study is to evaluate the incidence of IE in all types of surgical or transcatheter pulmonary valve implantations performed at a single institution. The study was a retrospective cohort study comprising all patients who underwent surgical right ventricle (RV) to pulmonary artery (PA) conduit (Contegra or homograft) or pulmonary valve implantation (Mosiac or Carpentier) between January 2010 to March 2020 and transcatheter pulmonary valve implantation (Melody or Sapien XT) between February 2007 to January 2020 at our institution. Patients with failed transcatheter valve implantation or who experienced immediate postoperative death were excluded from the study. The period at risk for endocarditis was defined as the time between the insertion of the conduit/valve until the most recent cardiac follow-up, conduit/valve explant, or IE diagnosis. The study's primary endpoint was to assess and compare the incidence of the first episode of endocarditis during the study period. A total of 134 RVOT reconstruction surgeries were performed in 119 patients: 62 Contegra conduits in 49 patients, 26 homografts in 24 patients, 35 Mosaic valves in 35 patients, and 11 Carpentier valves in 11 patients. 172 transcatheter pulmonary valve implantations were performed in 162 patients: 152 Melody valves in 142 patients and 20 Sapien XT valves in 20 patients. Only 7 transcatheter valve patients had a gradient $>20 \mathrm{mmHg}$ immediately after implantation. There were 22 cases of IE in the entire cohort, none related to homograft, Mosaic, and Carpentier valves. The incidence of IE in the Sapien XT valve, Melody valve, and Contegra conduit was $1.8,2.5$, and 4.9 per 100 patient-years, respectively. A definite source was identified as cat exposure (Bartonella endocarditis) in 1 patient and dental caries in 4 patients. Four patients had conduit/valve vegetation identified by transthoracic echocardiogram. Six patients required surgical conduit/valve replacement, three each with Melody valve and Contegra conduit. Both Melody and Contegra groups had a significant increase in the peak RVOT gradient at the time of diagnosis compared to prior imaging. The Hazard ratio (HR) for developing IE in Contegra conduit was 2.33 compared to Melody valve (95\% confidence interval $(\mathrm{CI}) 0.94-5.75, \mathrm{p}=0.066)$. A history of $\geq 2$ prior RVOT conduit or pulmonary valve implantation was associated with risk of developing IE, HR 2.8 (95\% CI 1.09-6.07), $\mathrm{p}=0.031$ ). No other risk factors were identified, including male sex, genetic syndrome, or RVOT gradient after transcatheter pulmonary valve implantation. Transcatheter pulmonary valve or RV to PA conduit derived from bovine jugular vein predispose to developing IE. Although not statistically significant, Contegra conduits had a higher risk compared to the Melody valve. Repeated RVOT intervention was found to be a risk factor for IE.

\section{ETHICS DECLARATIONS}

Conflict of interest: The authors declare that they do not have any conflict of interests.

Ethical Approval: The study qualified for IRB exemption by the Western Institutional Review Board.

Consent for Publication: Not applicable.
041. Transcatheter Pulmonary Valve Implantation with the Alterra Adaptive Prestent and SAPIEN 3 Transcatheter Heart Valve: One-year Outcomes of the ALTERRA Pivotal Trial

Evan Zahn', Vivian Dimas ${ }^{2}$, Vasilis Babaliaros ${ }^{3}$, Dennis Kim ${ }^{3}$, Scott Lim $^{4}$, Gareth Morgan ${ }^{5}$, Thomas K. Jones ${ }^{6}$, Aimee K. Armstrong ${ }^{7}$, Darren Berman ${ }^{7}$, Jamil Aboulhosn ${ }^{8}$, Vaikom S. Mahadevan', Matthew Gillespie ${ }^{10}$, David Balzer ${ }^{11}$, Thomas Zellers $^{12}$, Girish Shirali ${ }^{13}$, Anitha Parthiban ${ }^{14}$, Jonathan Leipsic $^{15}$, Philipp Blanke ${ }^{15}$, Yanjun Chen ${ }^{16}$, Shabana Shahanavaz $^{17}$

${ }^{1}$ Cedars-Sinai Medical Center, Los Angeles, USA. ${ }^{2}$ Medical City Children's Hospital Heart Center, Dallas, USA. ${ }^{3}$ Structural Heart and Valve Center, Emory University, Atlanta, USA. ${ }^{4}$ University of Virginia, Charlottesville, USA. ${ }^{5}$ Children's Hospital, University of Colorado, Aurora, USA. 'Seattle Children's Hospital, Seattle, USA. ${ }^{7}$ Nationwide Children's Hospital, Columbus, USA.

${ }^{8}$ Ahmanson/UCLA Adult Congenital Heart Center, Los Angeles, USA. ${ }^{9}$ University of California, San Francisco, San Francisco, USA. ${ }^{10}$ Children's Hospital of Philadelphia, Philadelphia, USA.

${ }^{11}$ Washington University in St. Louis School of Medicine, St. Louis, USA. ${ }^{12}$ Children's Health Dallas, Dallas, USA. ${ }^{13}$ Children's Mercy Hospital, Kansas City, USA. ${ }^{14}$ Texas Children's Hospital, Baylor College of Medicine, Houston, USA. ${ }^{15}$ St. Paul's Hospital, Vancouver, Canada. ${ }^{16}$ Edwards Lifesciences, Irvine, USA.

${ }^{17}$ Cincinnati Children's Hospital, Cincinnati, USA

Due to the variability in size and morphology of the right ventricular outflow tract (RVOT) which affects placement of transcatheter pulmonary valves, the Alterra Adaptive Prestent was designed to internally reduce and reconfigure the RVOT and provide a stable landing zone for the $29 \mathrm{~mm}$ SAPIEN 3 transcatheter heart valve (THV) during the treatment of pulmonary regurgitation (PR) in patients with dysfunctional RVOT/pulmonary valve (PV). The 6-month primary endpoint of the ALTERRA Pivotal Trial was previously reported and demonstrated the safety and effectiveness of this system. Here, we present the 1-year follow-up data. Patients were eligible for enrollment in the single-arm, prospective, multicenter, pivotal trial if they had $\geq$ moderate $P R$ and weighed $\geq 20 \mathrm{~kg}$. Patients were also required to have anatomy suitable for implantation, including a landing zone length $\geq 35 \mathrm{~mm}$ and a landing zone diameter between 27 and $38 \mathrm{~mm}$. Parameters evaluated included need for RVOT reintervention and degree of PR and/or stenosis. Echocardiography and cardiac CT/MRI core laboratory evaluations were utilized to assess pertinent right heart hemodynamics, including degree of pulmonary and tricuspid regurgitation (TR), mean RVOT gradient, and right ventricular size and function. Categorical data are presented as frequency (\%) and continuous data as median (interquartile range). A total of 60 patients were implanted across 11 sites with $100 \%$ implant success. Median age at implant was 21.5 (16.0-44.0) years, $50 \%$ were $\leq 21$ years old, and $56.7 \%$ were male. The majority had Tetralogy of Fallot (70.0\%), followed by PV stenosis (23.3\%). A total of $54(90.0 \%)$ patients had a 1-year follow-up visit; however, at the time of abstract submission, echocardiographic and CT or MRI data were available for 51. Finalized results will be available at the time of the meeting. At 1 year, freedom from device failure, death, RVOT reintervention or surgery, and endocarditis was $100 \%$. The majority of patients $(90.0 \%)$ had $\leq$ mild PR, with none having severe total or paravalvular PR. Right-ventricular end-diastolic indexed volume decreased from $155.6(135.8-177.6) \mathrm{ml} / \mathrm{m}^{2}(\mathrm{n}=59)$ at baseline to $110.3(94.1-139.1) \mathrm{ml} / \mathrm{m}^{2}(\mathrm{n}=43)$. The mean RVOT gradient remained stable through 1 year, with a median of $8.4(6.3-11.7)$ mmHg. The percentage of patients in New York Heart Association 
class I improved from $53.3 \%$ at baseline to $88.9 \%$. TR progressed from mild at baseline to moderate in 5 patients without further progression through the first year. Only 2 of these cases were attributed to tricuspid injury during valve advancement. None warranted therapy. Alterra frame fractures were documented in 12 patients, all were identified at or prior to the 6-month visit, and none were associated with clinical sequelae or reintervention. Outcomes for the ALTERRA Pivotal Trial at 1 year continue to demonstrate the safety and effectiveness of transcatheter pulmonary valve implantation with the Alterra Adaptive Prestent and SAPIEN 3 THV in patients with dysfunctional RVOT/PV requiring treatment for PR.

\section{ETHICS DECLARATIONS}

Conflict of interest: NPI, Consultant and Proctor, Edwards Lifescience.

Ethical Approval: All centers ITB approved.

Consent for Publication: Not applicable.

\section{Outcomes After Direct Reimplantation of Anomalous Right Pulmonary Artery from the Ascending Aorta}

\section{Liliam Aquino, Saleem Almasarweh, Nao Sasaki}

Nicklaus Children's Hospital, Miami, USA

Anomalous right pulmonary artery from the ascending aorta (AORPAA) is a rare disease entity. Early surgical repair by direct reimplantation to decrease the right ventricular pressure and prevent the development of pulmonary vascular occlusive disease has excellent outcomes and a low incidence of reintervention. The freedom from reintervention at 20 years has been reported as high as $80 \%$. Our objective is to report a single-center outcome in our institution. We retrospectively reviewed all patients who underwent reimplantation of AORPAA between 1995 and 2020. Preoperative data, operative reports, and postoperative course were reviewed. A Kaplan-Meier curve representing the overall probability of freedom from reintervention is reported. A total of 9 patients underwent surgical repair of AORPAA in the study period. Six were males $(66 \%)$, all were full term, and the median birth weight was $3.4 \mathrm{~kg}$. The median age at the time of diagnosis was 8 days (range 1-126 days). All patients were diagnosed by echocardiogram, 4 patients had additional imaging, 2

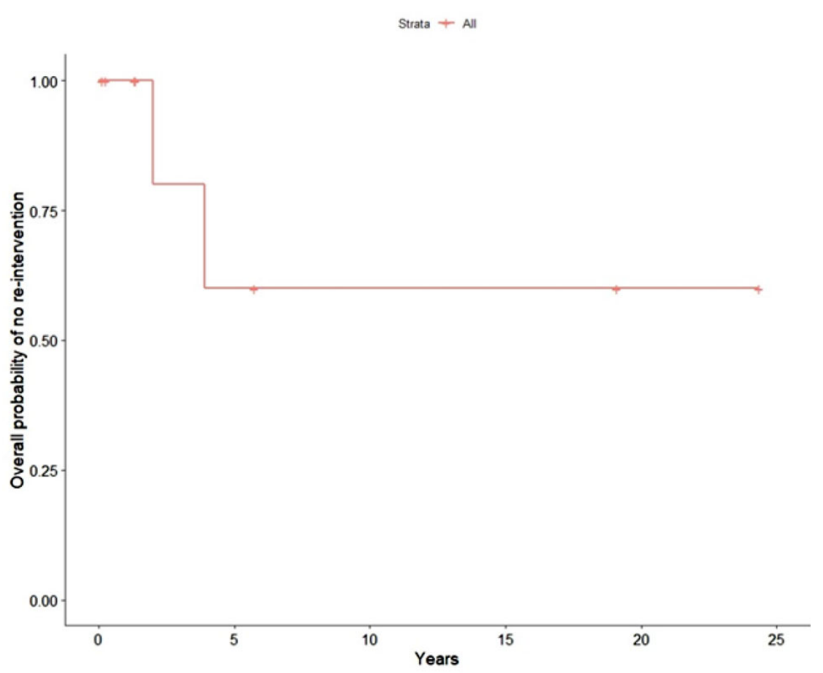

Fig. 1 Kaplan-Meier curve cardiac catheterizations, and 2 CTA. Three patients had significant additional lesions, one with coarctation of the aorta, and 2 had large perimembranous VSD. Seven patients had an associated PDA. Chromosome 22q11 deletion was detected in 3 patients (33\%). Direct reimplantation of AORPAA was performed in all patients with native tissue-to-tissue anastomosis without patch augmentation. The median operative age and weight were 10 days (range 6-147 days) and $3.9 \mathrm{~kg}$ (range $2.8-5.9 \mathrm{~kg}$ ), respectively. The median postoperative length of stay was 15 days (range 6-50 days) with no operative mortality. Follow-up data from all patients, representing 72 cumulative patient-years. No deaths or surgical reintervention occurred during the follow-up period. Seven patients were free of re-intervention after primary surgical repair. Two patients required catheter-based intervention, one patient with balloon angioplasty at 4 years after repair and one patient with stent angioplasty at 2 years after repair. At 20 years, freedom from reintervention by Kaplan-Meir estimate was $60 \% \pm 16.9 \%$ (Fig. 1). Direct reimplantation of AORPAA without patch augmentation has excellent outcomes. Pulmonary artery stenosis at the anastomosis site is a known complication requiring catheter-based intervention, but its incidence is low consistent with previous reports. Catheter-based reintervention is more likely in the first 5 years with 20 -year freedom of $60 \%$.

ETHICS DECLARATIONS

Conflict of interest: The authors declare that they do not have any conflict of interests.

Ethical Approval: The study qualified for IRB exemption by the Western Institutional Review Board.

Consent for Publication: Not applicable.

\section{Improving Efficiency in the Pediatric Cardiac Cath Lab: On-time Start of First Cases}

Emily Miller, Veronica Zitterman, Leanne Domzalski, Jeffrey Zampi, Wendy Whiteside

Michigan Medicine, Ann Arbor, USA

In the pediatric cardiac catheterization lab (PCCL), we observed a significant discrepancy between patient arrival in-room and the scheduled start time, with patients arriving in room an average of $27.3 \mathrm{~min}$ past scheduled start time, and with only $20 \%$ of cases starting on time. This delay in case start is due in part to inefficient pre-procedural processes and an overall culture of complacency for "on time" case starts across all team members. Late case start times create inefficient utilization of catheterization lab space, PCCL and anesthesia staff, and impact patient/parent satisfaction. Our aim, was to improve on-time first case starts, by decreasing the average number of minutes a patient arrives "in room" from scheduled start time, and increasing the percent of cases starting on time. Using a continuous quality improvement methodology, we mapped patient flow through the pre-procedural process focusing on targets for potential improved efficiency. Beginning in $8 / 2017$, a culture of on-time case starts was reinforced and expectations of first case start times relative to patient arrival were re-evaluated resulting in a change in scheduled case start times. New processes to improve patient flow through the pre-procedural area were then implemented with the goal of minimizing patient movement and instead bringing care and testing to the patient. Finally, standardization of pre-procedural evaluation/testing and changes to PCCL staffing were implemented. Case start time and inroom data points were collected via electronic medical records. Statistical process control charts were utilized to follow change in the outcome variable over time. We observed two stepwise improvements in case start times. First, after initial changes in scheduled start time and patient flow, time between patient in-room and scheduled start 
time decreased from an average of $27.3 \mathrm{~min}$ to $10.9 \mathrm{~min}$. The percent of cases starting on time improved from 20 to $52.7 \%$. Second, with implementation of standardized pre-procedural evaluation/testing and staffing changes, we saw an additional decrease from 10.9 to 4 min with $60.7 \%$ of cases arriving in room on time. This improvement in on-time case starts has been sustained over the following 3 years. Continuous quality improvement methodology, including systematic evaluation of processes, standardization, and changed culture and expectations surrounding case start times, has resulted in a significant and sustained improvement in on-time first case start times in the PCCL. Given the success of this improvement project, we have applied several of these interventions to improve case turn over time and in-room time for all cases.

\section{ETHICS DECLARATIONS}

Conflict of interest: The authors declare that they do not have any conflict of interests.

Ethical Approval: Not applicable.

Consent for Publication: Not applicable.

\section{Modified Medtronic Microvascular Plug Implantation to Reduce Excessive Pulmonary Blood Flow in Single Ventricle Patients with Systemic to Pulmonary Shunts}

\author{
Lauren Carlozzi, Thomas Jones, Troy Johnston, Agustin Rubio, \\ Brian Morray
}

Seattle Children's Hospital, Seattle, USA

Balancing systemic and pulmonary circulations in shunted single ventricle patients can be challenging. When medical and surgical methods do not work, transcatheter techniques such as the diablo stent can reduce the caliber of a surgical shunt. Small studies in animals and humans have described the use of a modified Medtronic Microvascular Plug (MVP) (Medtronic, Inc. Minneapolis, MN) to reduce the effective cross-sectional area of branch pulmonary arteries. This technique has not been described as a tool for reducing pulmonary blood flow in single ventricle patients with systemic to pulmonary shunts or ductal stents. To describe the technical and short-term outcomes of pulmonary blood flow restriction using a modified MVP in three patients with clinical evidence of excessive pulmonary blood flow following palliation with a systemic to pulmonary artery shunt or ductal stent. The MVP has a self-expanding nitinol frame in four sizes $(3 \mathrm{Q}, 5 \mathrm{Q}, 7 \mathrm{Q}, 9 \mathrm{Q})$, each with a polytetrafluoroethylene (PTFE) covering. Prior to deployment, perforations were created in the PTFE that would reduce the cross-sectional area of the vessel by approximately $50 \%$ when the device was implanted. Careful hemodynamic assessments performed before and after implantation determined the effectiveness of flow restriction. Informed consent was obtained from each family after discussing the off-label use of this device. Patient 1 was born with PA/VSD and underwent ductal stent placement on DOL 4. Patient 2 was born with d-TGA, VSD, hypoplastic RV and underwent arterial switch operation and VSD closure but required placement of a BT shunt to augment pulmonary blood flow. In both cases there was evidence of pulmonary over-circulation with high saturations, renal dysfunction, LV dysfunction and failure to wean from mechanical ventilation. Modified 5Q MVPs were placed in the shunts with sustained reductions in arterial saturation and increases in diastolic blood pressure. Patient 1 was anticoagulated with lovenox and patient 2 with a heparin infusion. There was no clinical evidence of device thrombosis. In both cases, the families elected to withdrawal care 1 month post procedure in the setting of multiple non-cardiac comorbidities. Patient
3 was born with an unbalanced AV canal defect and underwent stage 1 palliation with a $6 \mathrm{~mm}$ Sano shunt. The patient developed severe AV valve regurgitation (AVVR) and evidence of excessive pulmonary blood flow resulting in failure to wean from respiratory support and necrotizing enterocolitis. A modified 7Q MVP was placed in the shunt which allowed the patient to tolerate feeds and wean from respiratory support. The patient was anticoagulated with lovenox. Due to persistent symptomatic AVVR despite surgical repair, the patient underwent successful heart transplant. This is the first series to describe MVP modification and deployment to palliate symptomatic pulmonary over-circulation in single ventricle patients. In each case, the expected physiologic response to a decrease in pulmonary blood flow was observed. Two patients died due to accumulated non-cardiac comorbidities. In the third patient, the intervention was sufficient to improve the clinical status of the patient while waiting for heart transplant. Thrombus formation is a significant concern but was not clinically observed with adequate anticoagulation with either heparin or lovenox. The extent of modification necessary to adequately restrict blood flow is unclear. In this series, modifications were conservative to reduce risk of excessive restriction and thrombus formation. The MVP can be modified to function as a vascular flow restrictor to restrict pulmonary blood flow although more work is necessary to study anticoagulation and techniques for device modification.

ETHICS DECLARATIONS

Conflict of interest: The authors declare that they do not have any conflict of interests.

Ethical Approval: Not applicable.

Consent for Publication: Not applicable.

\section{Right Ventricular Outflow Tract Perimeter by Cardiac Magnetic Resonance Imaging: A New Paradigm of Value in Transcatheter Pulmonary Valve Replacement}

\section{R. Allen Ligon, Larry A. Latson, Mark Ruzmetov, Lazaro Hernandez}

Joe DiMaggio Children's-Memorial Healthcare Systems, Hollywood, USA

At our center, right ventricular outflow tract (RVOT) intervention has evolved via a data-driven model to encompass transcatheter, surgical, or hybrid palliative methodologies. The latter includes off-bypass surgical plication then transcatheter pulmonary valve replacement (PVR). A standardized algorithm for pre-procedure workup includes magnetic resonance imaging (MRI) to include evaluation of a proper intended valve site within the RVOT by perimeter (circumferential) measurement in end-diastole, rather than the traditional diameter dimensions. Our primary aim was to compare patients by MRIderived perimeter values to their palliative interventions performed (transcatheter versus surgical/hybrid methodologies). Retrospective review of patients undergoing PVR palliation with a primary indication of pulmonary regurgitation between January 2017 and January 2021. All MRI studies were performed on a 1.5-Tesla clinical scanner with the RVOT perimeter and diameters retrospectively measured in all study patients by an imaging and interventional staff member agreeing on the intended valve site within the RVOT. MRI imaging encompassed multiplane reconstruction from either contrast MRA (Twist) or three-dimensional steady-state free-precession (SSFP) electrocardiographic-gated and respiratory navigated sequences. Patients who underwent computed tomography due to non-compatible materials or underwent surgical palliation due to coronary 
compression were excluded. Outcomes of palliative interventions were outlined via descriptive analyses. A total of 37 patients underwent palliative PVR that met study criteria - 21 transcatheter, 7 surgical, and 9 hybrid. Median age at palliative intervention was 26 years (range 8-70). The mean end-diastolic perimeter of the transcatheter cohort was $88.9 \pm 8.7 \mathrm{~mm}$ and in the surgical/hybrid cohort measured $106.6 \pm 7.5 \mathrm{~mm}$ (Fig. 1A). The diameter measurements from the same identified intended valve site within the transcatheter cohort were median $24.7 \mathrm{~mm}$ (range 19.9-27.2) in the smaller dimension and $31.3 \mathrm{~mm}(27-38.1)$ in the larger. For the surgical/hybrid cohort, this was $26.3 \mathrm{~mm} \mathrm{(22.5-33.1)} \mathrm{and} 39.7 \mathrm{~mm}$ (36.2-48.6), respectively. For the transcatheter cohort, the median "circularized" diameter derived from the perimeter measurement (divided by $\pi$ ) was $27.7 \mathrm{~mm}$ (range 24.3-32.4). Notably, this correlated $(\mathrm{r}=0.93, \mathrm{p}<0.01)$ with the median diameter of the narrowest region during actual transcatheter RVOT balloon sizing (lateral imaging) of $27.1 \mathrm{~mm}$ (range 23.2-30.1) (Fig. 1B). After transcatheter valve implantation, the mean outer diameter of the implanted prosthesis was $26.7 \pm 2.2 \mathrm{~mm}$. In comparison, the median circularized diameter based on the MRI-perimeter the surgical/hybrid cohort was $33.5 \mathrm{~mm}$ (range 30.3-38). MRI-derived RVOT perimeter is useful in planning PVR palliation in terms of candidacy of transcatheter PVR $(\leq 98 \mathrm{~mm})$ versus the need for a surgical/hybrid approach $(\geq 99 \mathrm{~mm})$. The circularized diameter can be calculated from this metric and correlates with transcatheter RVOT balloon sizing. Given the vast array of RVOT morphologies necessitating PVR, calculating
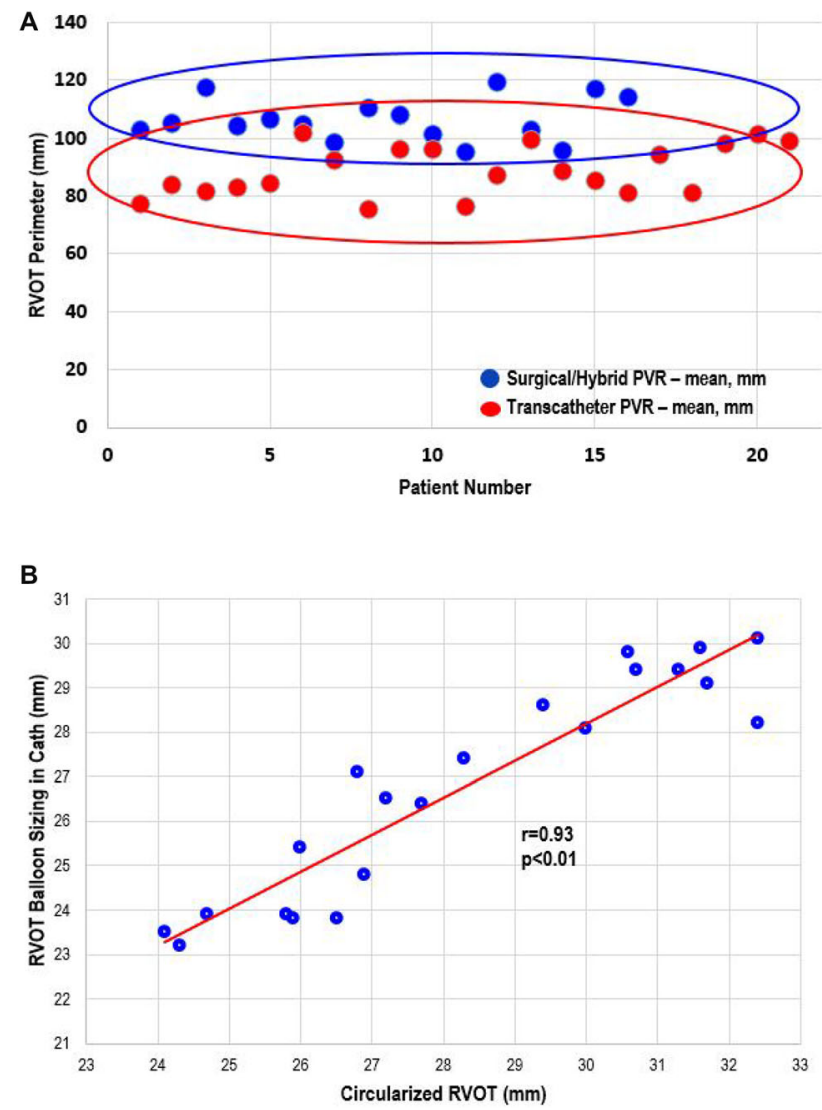

Fig. 1 Kaplan-Meier curve perimeter may prove more clinically beneficial than traditional measurements such as two-dimensional diameters.

ETHICS DECLARATIONS

Conflict of interest: The authors declare that they do not have any conflict of interests.

Ethical Approval: Not applicable.

Consent for Publication: Not applicable.

\section{Neonatal Myocardial Infarction: A Proposed Algorithm for Coronary Arterial Thrombus Management}

Hannah El-Sabrout, Srujan Ganta, Kanishka Ratnayaka, Gabrielle Vaughn, Amy Kimball, John Nigro, Howaida El-Said

Rady Children's Hospital/University of California, San Diego, San Diego, USA

Neonatal myocardial infarction (MI) is rare and associated with a high mortality of $40-50 \%$. We reviewed our experience with neonatal myocardial infarction, including presentation, management, outcomes, and the application of our current algorithm in managing these patients. The charts of newborns ( 1 day to 3 months) admitted to the neonatal intensive care unit (NICU) with a diagnosis of coronary artery thrombosis, coronary ischemia, and myocardial infarction between January 1, 2015 and May 31, 2021 were reviewed. 21 patients were identified. Thirteen $(62 \%)$ male and $8(38 \%)$ female had a median age and weight at diagnosis $1 \pm 8.35$ days and $3.20 \pm 0.71 \mathrm{~kg}$, respectively. Presentation was respiratory distress (16), shock (3), and murmur (2). Three had associated congenital heart disease (total anomalous pulmonary venous return, ventricular septal defect and coarctation of the aorta with a ventricular septal defect). Regional wall abnormality was noted in all 21 with varying degrees of depressed LV function [severe (8), moderate (6), mild (2) and low normal (5)]. EF was reported in $15 / 21$ with range of $20-51 \%$ (median 38\%). Mitral regurgitation was present in 19 (90\%) [mild (10), mild to moderate (2), moderate (6), moderate to severe (1)]. Left atrial hypertension was noted in $15(71 \%)$ and pulmonary hypertension in $18(86 \%)$. EKG was abnormal in 17 (81\%). Median Troponin was 0.18 (range 0.01-8). Median BNP 2100 (range 244 to $>5000$ ). Four patients had an MRI, all of which showed delayed enhancement. The etiology was unclear. All patients were treated based on our institutional management algorithm. Seventeen had documented coronary thrombosis by cardiac catheterization [LAD (4), Circ (3), $\mathrm{LAD}+\mathrm{Circ}(3), \mathrm{LMCA}+\mathrm{LAD}+\mathrm{Circ}(3), \mathrm{LAD}+\mathrm{RCA}$ (2), LMCA + LAD (1), LMCA + Circ + RCA 1). Seventeen of these patients $(81 \%)$ were treated with intracoronary TPA + systemic heparin + AT3 + IV nitroglycerin, and 4 (19\%) were treated with systemic heparin + AT3 + IV nitroglycerin alone. Fourteen (70\%) required inotropes, $16(80 \%)$ required a ventilator, and $5(25 \%)$ required INOs. Nineteen patients recovered. One patient died (this patient also had infra-diaphragmatic-TAPVR). One patient required a VAD and had a heart transplant; this patient presented late at 5 weeks of age and did not respond to TPA. Nineteen recovering patients returned to normal LV function with an EF of $60-74 \%$ (median $65 \%$ ) at latest follow up [median 6.8 months (range 19 days to 4.4 years)]. Two had residual trivial mitral regurgitation. Using our institutional neonatal coronary artery thrombus management algorithm, our institution experienced a much lower mortality rate than that reported in the literature. Using this algorithm may lead to improvement in patient outcomes following coronary arterial thrombus. 


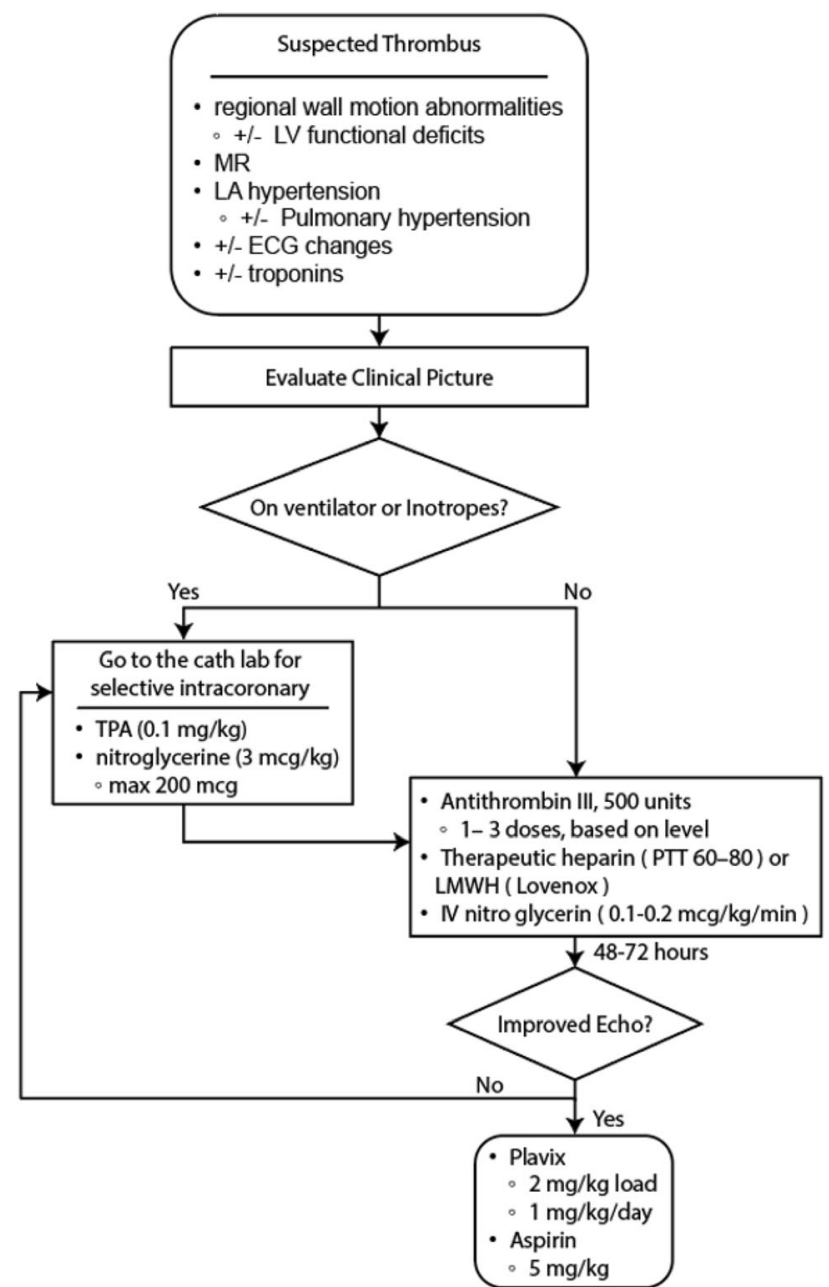

ETHICS DECLARATIONS

Conflict of interest: KR is an inventor of technology, assigned to National Institutes of Health, for transcatheter Glenn and Fontan procedures (US20170071722A1). Transmural Systems: collaborator (KR). Transmural Systems did not fund or support the research in this manuscript.

Ethical Approval: Not applicable.

Consent for Publication: Not applicable.

\section{A Novel Transcatheter Repair of Double Chamber Right Ventricle by Radio-Frequency Muscle Resection}

\section{Andrew Reittinger, Scott Lim, Nishtha Sodhi, Dale Fowler \\ University of Virginia, Charlottesville, USA}

Double-chambered right ventricle (DCRV) is a progressive hypertrophy of right ventricular (RV) muscle bundles causing obstruction at the right ventricular outflow tract (RVOT), typically associated with perimembranous ventricular septal defects (VSD). ${ }^{1}$ The current treatment strategy is surgical resection of the obstructive muscle bundle for patients who are symptomatic. ${ }^{2}$ There are currently no definitive treatment options for patients with DCRV that are not surgical candidates. Transcatheter modification of left-sided obstructive lesions using radiofrequency cutting wires has been developed in high-surgical risk patients, and therefore we proposed using these techniques for a patient at high-surgical risk with DCRV. A 73 year-old female with symptomatic, severe DCRV and a small perimembranous VSD, complicated by obstructive lung disease, presented with worsening dyspnea. Gradient by transthoracic echocardiography across the RVOT was $>100 \mathrm{mmHg}$, and by diagnostic catheterization proximal right ventricle (RV) was 120/16 compared to pulmonary pressure of $46 / 12$, with no oximetric shunt detected. Following review by the multi-disciplinary heart team, she was felt to be high risk for surgery based on age and comorbidities, and she consented for catheter-based intervention. For the procedure, a radiofrequency wire was advanced under the guidance of transesophageal echocardiography and angiography through the obstructing RVOT muscle bundles, and snared in the pulmonary artery (see Figure). Using $50 \mathrm{~J}$ of energy the bundles were cut in a single pass. After intervention, the RV pressure decreased to $54 / 14 \mathrm{mmHg}$, with RVOT gradient less than $10 \mathrm{mmHg}$, and no significant left-to-right shunt detected by oximetry. The patient at 1 month follow-up has had resolution of her symptoms. Transcatheter radiofrequency resection of muscle bundles obstructing the right ventricular outflow tract associated with DCRV was safe and effective in this high-risk surgical candidate. This opens up a potential novel treatment paradigm for DCRV.

Cohen MS, Lopez L. Chapter 30: Ventricular Septal Defects. In: Allen HD, Shaddy RE, Penny DJ, Feltes TF, Cetta F eds. Moss and Adams' Heart Disease in Infants, Children, and Adolescents. 9th ed. Wolters Kluwer. 2016:784-802.

McElhinney DB, Chatterjee KM, Reddy VM. Double-chambered right ventricle presenting in adulthood. Ann Thorac Surg. 2000 Jul;70(1):124-7. https://doi.org/10.1016/s0003-4975(00)01320-5. PMID: 10921695.

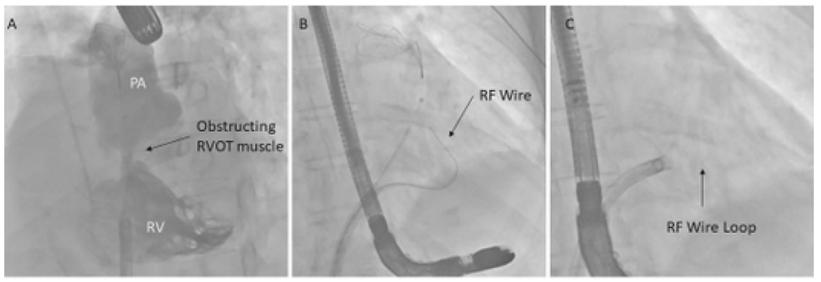

ETHICS DECLARATIONS

Conflict of interest: The authors declare that they do not have any conflict of interests.

Ethical Approval: Not applicable.

Consent for Publication: Not applicable.

\section{Identical Cardiac Lesions with Divergent Physiology in a Monozygous/Diamniotic Pregnancy}

\author{
Ugonna Nwankwo ${ }^{1}$, Chetana Reddy ${ }^{1}$, Beth Hill ${ }^{2}$, Amy Astudillo ${ }^{2}$ \\ ${ }^{1}$ Saint Louis University, St. Louis, USA. ${ }^{2}$ SSM Cardinal Glennon, St. \\ Louis, USA
}

This case study reviews a monozygous/diamniotic pregnancy resulting in twins with identical cardiac lesions with divergent physiology. Fetal Care patient with complications including monozygous/diamniotic pregnancy, Trisomy 21 in both twins, gestational diabetes, and growth restriction of Twin A. The fetal ECHO on Twin A showed Tetralogy of Fallot and arch hypoplasia. The fetal ECHO on Twin B showed Tetralogy of Fallot. The twins were born at 30 weeks gestation. The Apgars on twin A were 6 and 7, Apgars on twin B were 7 and 8. Postnatal echocardiogram for Twin A showed moderately sized perimembranous VSD with bidirectional flow, appears mostly 
covered by tricuspid valve tissue. Small to moderately sized PDA with bidirectional shunting. PFO vs secundum ASD with left to right flow, no left heart enlargement. Mild tricuspid regurgitation. Normal biventricular systolic function. Postnatal echocardiogram for Twin B showed moderately sized perimembranous VSD $(3.5 \mathrm{~mm})$ with bidirectional flow, partially covered by tricuspid valve tissue. Moderately sized PDA with bidirectional shunting. Patent foramen ovale vs secundum atrial septal defect with mostly left to right flow, no left heart enlargement. Mild tricuspid regurgitation. Normal biventricular systolic function. Both twins spent 3 months in the NICU mostly due to feeding difficulties resulting in a G-tube placement for both twins and respiratory failure. Both twins had been started on Lasix while in the NICU for clinical signs of pulmonary overcirculation. Both twins failed weaning from respiratory support due to desaturations and were discharged on $1 / 8 \mathrm{~L}$ nasal cannula. At their first outpatient clinic visit, both twins had low velocity flow across their ventricular septal defects and a soft murmur on exam concerning for elevated right heart pressures. A catheterization was completed on each twin with very divergent findings. The catheterization findings on Twin A showed a moderate perimembranous VSD, moderate secundum ASD, mildly elevated RVEDP $(11 \mathrm{mmHg})$, elevated PA pressure (40-42 mmHg), normal PVR (1.92 iWU), upper normal LVEDP $(10 \mathrm{mmHg}), \mathrm{Qp}: \mathrm{Qs}=4.25: 1$. The findings on Twin B showed a large perimembranous VSD with baseline Qp:Qs 2.27:1, elevated pulmonary vascular resistance at baseline 5.7 Woods units $\mathrm{x}$ $\mathrm{m} 2$, pulmonary vasodilator testing with iNO $40 \mathrm{ppm}$ and $\mathrm{FiO} 260 \%$ demonstrated improvement in PVR to high normal level 2.7 Woods units $\times \mathrm{m}^{2}$ and lowered normal cardiac output $2.4 \mathrm{~L} / \mathrm{min} / \mathrm{m}^{2}$. Based on catheterization twin A underwent surgical intervention closure of VSD, closure of ASD, and ligation of the ductus. Based on catheterization Twin B was started on Tadalafil prior to the operation. The surgical intervention completed was closure of an ASD and VSD and ligation of the ductus. The post-operative course for both twins was uncomplicated. Both twins had identical cardiac lesions, echocardiogram findings with low velocity shunting across the ventricular septal defect with a soft murmur. Their cardiac cath hemodynamics were drastically different with Twin A with a Qp:Qs of 4.25:1 and significant pulmonary overcirculation and Twin B with an elevated PVR at baseline that was reactive to pulmonary vasodilators and started medication prior to surgery.

ETHICS DECLARATIONS

Conflict of interest: The authors declare that they do not have any conflict of interests.

Ethical Approval: Not applicable.

Consent for Publication: Not applicable.

\section{Selective Valve Removal for Melody Valve Endocarditis: Practice Variations in a Multicenter Experience}

Arpine Davtyan $^{1}$, Peter Guyon ${ }^{1}$, Hannah El-Sabrout ${ }^{2}$, Nanda Ramchandar $^{1}$, Reid Ponder ${ }^{2}$, Rachel Weber ${ }^{1}$, Wagih Zayed ${ }^{1}$, Linda Drake ${ }^{3}$, Kanishka Ratnayaka ${ }^{1}$, John Nigro' ${ }^{1}$, John Moore ${ }^{1}$, Holly Bauser-Heaton ${ }^{4}$, Laith Alshawabkeh ${ }^{5}$, Ryan Reeves ${ }^{5}$, Daniel Levi ${ }^{2}$, Jamil Aboulhosn ${ }^{2}$, Henri Justino ${ }^{3}$, John Bradley ${ }^{1}$, Howaida El-Said ${ }^{1}$

${ }^{1}$ UCSD/Rady Children's Hospital, San Diego, USA. ${ }^{2}$ UCLA/Mattel Children's Hospital, Los Angeles, USA. ${ }^{3}$ Baylor University/Texas Children's Hospital, Houston, USA. ${ }^{4}$ Emory University/Children's Healthcare of Atlanta, Atlanta, USA. ${ }^{5}$ UCSD, San Diego, USA
To identify factors associated with surgical valve removal versus antimicrobial therapy in Melody transcatheter pulmonary valve (TPV) infective endocarditis (IE). Guidelines for management of Melody TPV IE are lacking. Multicenter retrospective analysis of all patients receiving Melody TPV from 10/2010 to 3/2019 was performed to identify cases of IE. Surgical explants versus non-surgical cases were compared. Of the 663 Melody TPV, there were 66 cases of IE in 59 patients $(59 / 663,8.8 \%)$. 39/66 (59\%) were treated with IV antimicrobials; 27/66 (41\%) underwent valve explantation. 26/59 patients (44\%) were treated medically without explantation or recurrence with average follow up time of 3.5 years (range 1-9). 32\% of Streptococcus cases, $53 \%$ of MSSA and all MRSA cases were explanted. 2 of the 4 deaths had MSSA. CART analysis demonstrated two important parameters associated with explantation: a peak echo gradient $\geq 47 \mathrm{mmHg}$ at IE diagnosis (OR 10.6, p < 0.001) and an echo gradient increase of $>24 \mathrm{mmHg}$ compared to echo before diagnosis (OR 6.7, p = 0.01). Rates of explantation varied by institution $(27 \%$ to $64 \%$ ). In our multicenter experience, $44 \%$ of patients with Melody IE were successfully medically treated without valve explantation or recurrence. The degree of valve stenosis at time of IE diagnosis was strongly associated with explantation. Rates of explantation varied significantly among the institutions.

ETHICS DECLARATIONS

Conflict of interest: The authors declare that they do not have any conflict of interests.

Ethical Approval: Not applicable.

Consent for Publication: Not applicable.

\section{Experience of Percutaneous Transcatheter Valve Replacement in Pediatric Patients After CHD Surgical Correction}

Manolis Pursanov, MD PhD, Mikhail Abramyan, MD PhD, Alexey Bedin, MD, Davron Pardaev, MD, Yuriy Shamrin, MD, Maria Kurako, MD, Vladimir Chagirev, MD, Elena Talalaeva, MD

Moscow State Budgetary Healthcare Institution «Morozov Children's City Clinical Hospital of the Moscow City Healthcare Department», Moscow, Russian Federation

Percutaneous transcatheter valve replacement (PTVR) using Melody (Medtronic) and Sapien XT Edwards LifeSciences in pulmonary or tricuspid position has become a standard procedure after CHD surgical correction. In Russian pediatric practice these procedures have been performed in a small number of patients with CHD, and in cases after correction using transannular patch-has not been performed at all. During the period from October 2019 to March 2021 we performed 6 PTVR procedures in 6 patients with a history of various CHD corrections: in 5 cases in pulmonary position (4-Sapien XT, 1 -Melody Medtronic), and in one case in degenerated tricuspid bioprosthesis (Melody Medtronic). Pulmonary PTVR was performed after pre-stenting in order to prepare space for prosthesis fixation. Sapien XT was used in pulmonary artery (PA) diameter of 23 to $29 \mathrm{~mm}$, while Melody Medtronic of 16 to $22 \mathrm{~mm}$; the diameters were measured by balloon catheter when coronary arteries were not compressed by inflated balloon. All patients selected for pulmonary PTVR had a history of tetralogy of Fallot radical correction: transannular patch repair $(n=3)$; multistage correction with PA stenosis $(n=1)$; for absent pulmonary valve (12 $\mathrm{mm}$ monoleaflet mechanical valve implantation) and concominant left PA hypoplasia $(\mathrm{n}=1)$. The 2 least mentioned patients had also undergone stenting of PA and left PA. Patients weighed from 36 up to 56 kilos, aged 10 to 17 years. The 
diameter of Sapien XT was of $26 \mathrm{~mm}$ in 3 cases, and $29 \mathrm{~mm}$ in 1 case; Melody Medtronic — of $22 \mathrm{~mm}$. After PTVR into the pulmonary position no regurgitation was detected and peak systolic pressure gradient did not exceed $17 \mathrm{mmHg}$. Transcatheter tricuspid "valve-invalve" replacement was performed in 14-year-old patient, who had undergone one and-a-half ventricle repair (bidirectional cavopulmonary shunt + prosthetic replacement of tricuspid valve with Mosaic Medtronic No. 25) at the age of 1 year for Ebstein's anomaly. The patient had bioprosthetic valve disfunction: peak systolic pressure gradient of $29 \mathrm{mmHg}$ and severe tricuspid regurgitation, right heart dilatation, right ventricle ejection fraction of $18 \%$. Transcatheter tricuspid "valve-in-valve" replacement using Melody $22 \mathrm{~mm}$ was successfully performed after the pre-stenting. After the procedure the peak systolic pressure gradient did not exceed $3 \mathrm{mmHg}$, without regurgitation. All patients received antimicrobial profilaxys for 2-3 days after the intervention and subsequent low-dose aspirin therapy (3-5 mg/kg/day) for 6 months. Patients were discharged on the 3-4th day after the procedure. Transcatheter valve replacement in pulmonary or tricuspid position may be considered as an alternative to the open heart surgery due to the satisfying clinical and hemodynamic effects, if patients are selected correctly.

\section{ETHICS DECLARATIONS}

Conflict of interest: The authors declare that they do not have any conflict of interests.

Ethical Approval: Not applicable.

Consent for Publication: Not applicable.

\section{Outcomes Following Stent Implantation of Aortic Arch and isthmus Obstructions}

\section{Manolis Pursanov, MD, PhD, Mikhail Abramyan, MD, PhD, Alexey Bedin, MD, Davron Pardaev, MD, Yuriy Shamrin, MD, Maria Kurako, MD, Elena Talalaeva, MD}

Moscow State Budgetary Healthcare Institution «Morozov Children's City Clinical Hospital of the Moscow City Healthcare Department», Moscow, Russian Federation

During the period from November 2018 to March 2021 stent implantation into the aortic isthmus was performed in 27 patients using 28 stents. The mean age of patients was $10.3 \pm 7.6$ years, from 4 months up to 31 years). The mean weight was $\overline{35.7} \pm 21.4$ (7-84) kilos. In the analyzed group the procedure was performed for isolated native coarctation of the aorta $(n=18)$ and recoarctation $(n=6)$, including 3 after CHD repair. Gothic aortic arch stenting was performed twice. Various balloon expandable vascular stents were used: «P» $(\mathrm{n}=2)$, «Covered CP $(\mathrm{n}=8)$, «IntraStent LD Max» $(\mathrm{n}=2)$, «Intrastent LD Mega» $(n=4)$, «Palmaz-Genesis XD» $(n=3)$, Bentley $(n=1)$, AndraStent $(n=5)$, «Valeo» $(n=3)$. In the majority of cases the percutaneous approach was used, however in 4 cases arteriotomy was preferred. Immediately after the stenting peak systolic gradient between the ascending and descending aorta decreased from $47.7 \pm 13.7$ to $3.2 \pm 5.5 \mathrm{mmHg}$. All stent implantations were considered successful: peak systolic gradient did not exceed $20 \mathrm{mmHg}$ or was not detected. Two patients developed complications after the procedure: in one case during the first $24 \mathrm{~h}$ aortic dissection with hemothorax which required Covered $\mathrm{CP}$ implantation for urgent perforation closure and thrombotic mass removal from the left pleural cavity on the 3rd day. In another case an episode of ventricular fibrillation was detected during stenting of recoarctation in patient who had previously undergone the Norwood procedure, 4 days after successful intervention the patient died from resistant to treatment arrhythmia. Long- term follow-up (observation period 6-28 months). In three patients balloon dilatation of previously implanted stents was successfully performed as planned because of child's natural growth. Late complications related to the stent implantation were not registered. Stent therapy for coarctation of the aorta is an effective method for the management of the obstruction and may be considered as an alternative to the open-heart surgery.

ETHICS DECLARATIONS

Conflict of interest: The authors declare that they do not have any conflict of interests.

Ethical Approval: Not applicable.

Consent for Publication: Not applicable.

\section{Transcatheter Closure of Perimembranous Ventricular Septal Defects Using Different Generations of Amplatzer Devices: Multicenter Experience}

Roberto Mijangos Vázquez ${ }^{1}$, Rogelio Hernández Reyes ${ }^{1}$, Amal El-Sisi $^{2}$, Carlos Zabal ${ }^{3}$, José Antonio García Montes ${ }^{3}$, Juan Pablo Sandoval $^{3}$, Rodina Sobhy ${ }^{2}$, Antoine Abdelmassih ${ }^{2}$, Mohammed Soliman', Safaa $\mathrm{Ali}^{4}$

${ }^{1}$ Pediatric Specialties Hospital, Tuxtla Gutiérrez, Mexico. ${ }^{2}$ Cairo University Children Hospital, Cairo, Egypt. ${ }^{3}$ National Institute of Cardiology "Ignacio Chávez", Mexico, Mexico. ${ }^{4}$ Sohag University Hospital, Sohag, Egypt

To demonstrate safety and efficacy of using different generations of softer Amplatzer ${ }^{\mathrm{TM}}$ devices for ventricular septal defect (VSD) closure to avoid serious complications at follow-up. Transcatheter closure of perimembranous ventricular septal defects (PmVSD) is a well-established procedure; however, it is associated with unacceptable incidence of complete heart block. Great advantages have been achieved by using softer devices for VSD transcatheter closure..e first and second generation of Amplatzer ${ }^{\mathrm{TM}}$ occluders (AVP II, ADO, and ADO II) seem to offer a safe and attractive alternative for this procedure. These devices can be delivered using either an arterial (retrograde) or venous (prograde) approach. Patients with congenital PmVSD who underwent transcatheter closure using ADO, ADO II, and AVP II devices were included. Primary end point was to determine efficacy and safety of these generations of devices and to determine the incidence of complications at follow-up (complete AV block and aortic/tricuspid/mitral regurgitation). One hundred and nineteen patients underwent VSD closure at a median age of 5 years ( 8 months to 54 years). During the catheterization, there were only minor complications and at follow-up of $36 \pm 25.7$ months (up to 99 months), the closure rate was high of $98.3 \%$ and freedom from AV block was $100 \%$. Conclusions: The use of softer Amplatzer ${ }^{\mathrm{TM}}$ devices is a good alternative to achieve PmVSD closure safely with no risk of AVB during the procedure or at midterm follow-up.

\section{ETHICS DECLARATIONS}

Conflict of interest: The authors declare that they do not have any conflict of interests.

Ethical Approval: Not applicable.

Consent for Publication: Not applicable. 
053. Percutaneous Closure of Patent Ductus Arteriosus in Premature Infants: Single Center Experience Comparing the Use of Multiple Devices

Peter Guyon ${ }^{1}$, Nicole Duster ${ }^{2}$, Anup Katheria ${ }^{3}$, Caitlin Heyden ${ }^{1}$, Kanishka Ratnayaka $^{1}$, Howaida El-Said ${ }^{1}$

${ }^{1}$ Division of Pediatric Cardiology, Rady Children's Hospital and UC San Diego School of Medicine, San Diego, USA. ${ }^{2}$ Department of Pediatrics, Rady Children's Hospital and UC San Diego School of Medicine, San Diego, USA. ${ }^{3}$ Department of Neonatology, Sharp Mary Birch Hospital for Women and Newborns, San Diego, USA

Innovative techniques and novel devices have led to successful percutaneous closure of patent ductus arteriosus (PDA) in increasingly small premature infants. Our center has successfully used the Microvascular Plug, "MVP" (Medtronic, Minneapolis, MN); Micro Plug Set, "Micro Plug" (KA Medical, Minneapolis, MN); and Amplatzer Piccolo Occluder, "Piccolo" (Abbot, Santa Clara, CA). We report our experience and compare demographic factors and procedural outcomes between patients grouped by device selected for PDA closure. This is a retrospective review of all ex-premature infants who underwent percutaneous closure of PDA at a single center (June 2018 to May 2021). Patients were grouped based on the initial device selected for PDA closure (intention to treat analysis). ANOVA (analysis of variance) was used to compare numerical data; KruskalWallis Rank Sum Test was used to compare ordinal data. Statistical analysis was performed using $\mathrm{R}$ version 4.0.2 ( $\mathrm{R}$ Foundation for Statistical Computing, Vienna, Austria). 58 premature infants underwent successful percutaneous PDA closure (mean gestational age $27 \mathrm{w} 2 \mathrm{~d}$; mean weight $990 \mathrm{~g}$; mean age at procedure 29 days). Groups based on device were: MVP $(n=25)$, Micro Plug $(n=25)$, and Piccolo $(\mathrm{n}=8)$. Pre-procedural demographics (gestational age, birth weight, procedure weight, sex, respiratory support) and procedural data (PDA size, fluoroscopy time, radiation, time to extubation) were similar between the three groups. One MVP device was deployed (not released) then retrieved for sub-optimal fit and changed to a Micro Plug device. Two Piccolo devices were also retrieved and changed to MVP devices for the same reason. None of the Micro Plug devices were retrieved/exchanged. Three devices (2 MVP, 0 Micro Plug, 1 Piccolo, $\mathrm{p}=0.28$ ) embolized in the immediate post-procedure setting. All three devices were retrieved successfully using a snare and angled glide catheter. The two patients with embolized MVPs were referred to surgery after retrieval, while the third (Piccolo) patient had successful placement of an MVP during the retrieval procedure. One MVP was removed two days post procedure for partial obstruction of the aorta with a gradient by echo; after retrieval the PDA spontaneously closed. There was one mortality: in addition to PDA the patient had Beckwith Weidman Syndrome and ruptured omphalocele with recurrent episodes of sepsis and renal failure. 10 days after transcatheter PDA closure, embolization, then successful surgical closure, the patient's parents withdrew life support for intractable multi-system organ failure. Device selection evolved over time. Initially, the MVP was used exclusively. In late 2019 and early 2020, the Piccolo was introduced. Our program quickly transitioned to the Micro Plug device once it was introduced in early 2020, and since September 2020 (15 consecutive cases), the Micro Plug has been used exclusively. PDA closure in premature infants was performed safely using MVP, Piccolo, and Micro Plug devices. Demographic and procedural factors were similar between the groups. In contrast to the other devices, the Micro Plug did not require exchange for sub-optimal fitting or embolize (not statistically significant). Our center has evolved to exclusive use of the Micro Plug device for premature infant PDA device closure.
Conflict of interest: The authors declare that they do not have any conflict of interests.

Ethical Approval: The study was IRB approved with a waiver of consent.

Consent for Publication: Not applicable.

\section{Transcatheter Stent Placement Between the Left Innominate Artery and the Left Pulmonary Artery in a Newborn with Tetralogy of Fallot and Disconnected Left Pulmonary Artery}

\author{
Josue Diaz-Frias $^{1}$, Ahmad Khalil ${ }^{1}$, Yasser Al-Khatib ${ }^{1,2}$, Enrique \\ Oliver Aregullin 1,2 \\ ${ }^{1}$ Congenital Heart Center, Spectrum Health Helen DeVos Children's \\ Hospital, Grand Rapids, USA. ${ }^{2}$ Pediatrics and Human Development, \\ Michigan State University College of Human Medicine, Grand \\ Rapids, USA
}

Disconnected pulmonary artery or ductal origin of the pulmonary arteries is a rare congenital defect with an estimated incidence of 1 in 200,000 as an isolated anomaly and a prevalence of $0.4 \%$ when associated with other congenital malformations. Failure of early detection and management leads to intraparenchymal pulmonary arterial hypoplasia, formation of aortopulmonary collateral vessels, and increased risk for pulmonary infections, hemorrhage, pulmonary hypertension, thoracic asymmetry and scoliosis. We describe the case of an 11-day-old newborn initially referred to the cardiology clinic for evaluation of a murmur who was found to have tetralogy of Fallot, right aortic arch and concerns for disconnected left pulmonary artery (LPA) on the initial echocardiogram. The patient underwent cardiac catheterization for intended transcatheter stenting of the diffusely narrow arterial duct between the left innominate artery and the LPA. We initially established wire connection from the stump of the left innominate artery to the distal LPA in a retrograde fashion with the aid of a 4 French 2.5 JR catheter and a 0.035 -inch glidewire, hand injection angiography confirmed adequate position (Fig. 1). Wire exchange was then done with 0.014-inch Prowater Ashashi wire. From retrograde approach through a $3 / 4$ french femoral artery sheath,

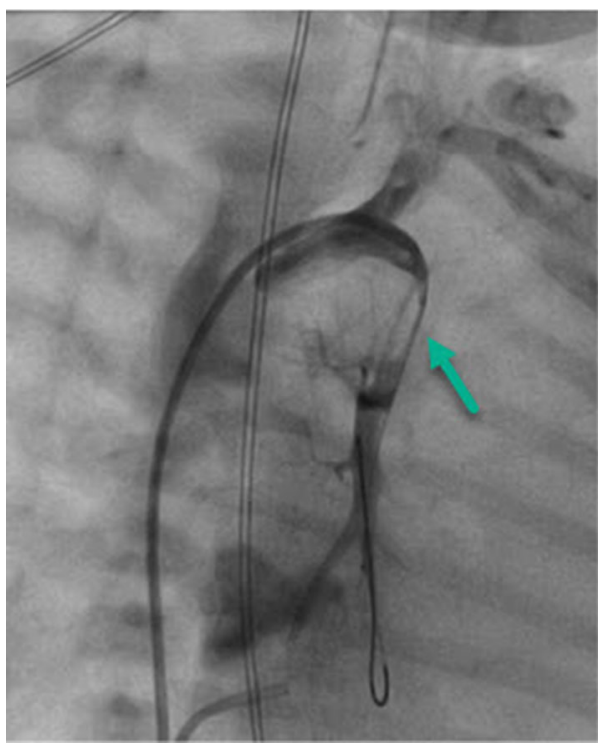

Fig. 1 . 


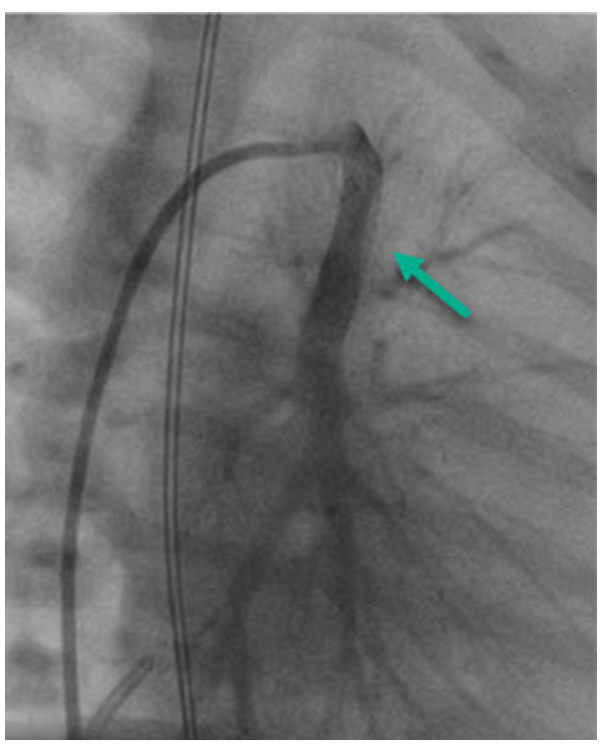

Fig. 2 .

an Integrity $3.5 \times 22 \mathrm{~mm}$ coronary stent was advanced and deployed, securing the connection between the left innominate artery and the intraparenchymal LPA. Post-intervention angiography showed excellent flow and stent position with adequate filling of the LPA, and normal pulmonary vein return (Fig. 2). Post-procedure course was complicated by left pulmonary edema that resolved in $48 \mathrm{~h}$, and low diastolic pressures with development of necrotizing enterocolitis that was managed conservatively. Patient underwent complete surgical repair sixteen days after catheter procedure due to persistent low diastolic blood pressure and occult blood on stool, with excellent hemodynamic and echocardiographic results and was discharged home fourteen days later.

Discussion: A disconnected pulmonary artery is a rare condition that can have deleterious complications if left untreated, hence, the importance of early recognition and intervention. Nevertheless, the diagnosis can present a challenge to clinicians, particularly in the setting of an isolated defect in which patients may be asymptomatic. Transcatheter arterial duct stenting has been described as an initial intervention that can aid re-implantation of the pulmonary artery in those patients with late diagnosis and significant pulmonary arterial hypoplasia. The ultimate therapeutic goal is to provide effective pulmonary blood flow to the affected vessel and help with growth stimulation. However, patients can have significant hemodynamic changes following the procedure, including the development of pulmonary edema or post-reperfusion injury, elevated right ventricular pressures and potential dilation of the right ventricle. Additionally, in the setting of a large ductal stent, there could be a significant diastolic run-off that could lead to systemic steal with relevant clinical repercussions. Overall, the management of a disconnected pulmonary artery requires a multidisciplinary strategy with close monitoring. The decision of transcatheter intervention should be weighted by the hemodynamic impact it will have in each case and the possible complications that may arise after this approach.

\section{ETHICS DECLARATIONS}

Conflict of interest: The authors declare that they do not have any conflict of interests.

Ethical Approval: Not applicable.

Consent for Publication: Not applicable.

\section{Multi-Disciplinary Airway Evaluation and Interventions in the Cardiac Catheterization Laboratory}

\author{
Nikita Mittal ${ }^{1}$, Peter Guyon ${ }^{1}$, Kanishka Ratnayaka ${ }^{1}$, Matthew \\ Brigger $^{2}$, Howaida El-Said ${ }^{1}$ \\ ${ }^{1}$ Division of Pediatric Cardiology, Rady Children's Hospital and UC \\ San Diego School of Medicine, San Diego, USA. ${ }^{2}$ Division \\ of Pediatric Otolaryngology, Rady Children's Hospital and UC San \\ Diego School of Medicine, San Diego, USA
}

Augmented imaging available in the cardiac catheterization lab (biplane X-Ray fluoroscopy and contrast bronchography) in addition to standard laryngoscopy and bronchoscopy has enhanced complex airway evaluation and allowed for multi-disciplinary interventions. We conducted a retrospective review of complex airway disease patients evaluated and treated in the cardiac catheterization lab over 3 years (September 2017 to May 2021). A multi-disciplinary complex airway team (interventional cardiology, cardiothoracic surgery, otolaryngology, and pulmonology) evaluated patients using bronchoscopy, laryngoscopy, X-Ray fluoroscopy, and contrast bronchography. Patients failing initial conservative management strategies or with treatment refractory complex anatomy were considered for rescue balloon dilation and airway stenting. Evaluation and treatment of complex airway disease with augmented imaging by a multi-disciplinary team in the cardiac catheterization lab was successful in all 11 patients attempted. There were no procedural adverse events and there were no complications at most recent follow up (mean 13 months, range 1-44 months). Airway stent implantation was performed in 6/11 cases; airway balloon dilation was performed in 3/11 cases; diagnostic fluoroscopy and bronchograms only (no intervention indicated) were performed in $2 / 11$ cases. Of the 6 airway stent patients, 5 bare metal stents [4 Integrity and 1 Visi-Pro (Medtronic, Minneapolis, MN)] were placed to relieve stenosis (mean increase from 54 to $99 \%$ post stenting of the appropriate airway diameter). One covered stent (Viabahn, W. L. Gore, Flagstaff, AZ) was placed to treat a large slit-like tracheoesophageal fistula. All patients had immediate clinical improvement and there were no stent-related complications such as embolization, migration, or erosion at most recent follow up. Two stents remain patent and well seated into surrounding tissue. One stent was safely removed after 13 months in situ; the patient is clinically well and there was resolution of bronchomalacia at stent site. Three patients died from unrelated complications. However, the most recent evaluation prior to death demonstrated patency with no evidence of airway complications in each patient. Of the 3 patients who underwent airway balloon dilation without stenting, 2 patients were initially referred for subglottic/tracheal stenosis and one for a tracheal diverticulum with chronic infections and stridor. In 1 tracheal stenosis patient, balloon dilation resulted in mild improvement from 53 to $64 \%$ of appropriate airway diameter. In the other patient, the result was graded qualitatively as "mild improvement" in stenosis. In the patient with tracheal diverticulum, an otolaryngology surgeon first made a membrane incision with sickle knife and microscissors. This was followed by balloon dilation of the stenotic area by the interventional cardiology team, resulting in immediate and on-going mucous clearance and improvement of symptoms. The 2 patients who underwent fluoroscopy and bronchograms only remained free from any complications at the time of most recent follow up. In 11 patients with complex airway disease, treatment in the cardiac catheterization lab by a multi-disciplinary complex airway team resulted in successful evaluation and safe interventions as indicated. This approach can likely serve as a model for other institutions in the management of complex airway disease. 
ETHICS DECLARATIONS

Conflict of interest: The authors declare that they do not have any conflict of interests.

Ethical Approval: Not applicable.

Consent for Publication: Not applicable.

\section{Hybrid Pulmonary Valve Replacement: Cleveland Clinic Experience}

Rida Shahid, Petcharapong Suntharos, Edgard Bendaly, Alistair Phillips

\section{The Cleveland Clinic, Cleveland, USA}

Surgical repair of conotruncal anomalies often results in pulmonary valve dysfunction which can lead to long term negative effects. Since its introduction in 2000, trans-catheter pulmonary valve replacement (tPVR) has shown to be a viable alternative to surgery especially in the high risk population. However, multiple factors limit the feasibility of tPVR such as right ventricular outflow tract (RVOT) dilatation with an unsuitable landing zone and small patient size. Prior to approval of Harmony valve, at our center, we had adopted the Hybrid procedure that combines the strengths of both the surgical and trans-catheter techniques, by avoiding cardiopulmonary bypass and complete median sternotomy. Through a limited sternotomy approach, either the MPA is externally banded to decrease the diameter of the landing zone or the valve apparatus is delivered through a ventriculotomy in younger patients and in patients with no viable vascular access. We present the characteristics and outcomes of all the patients that underwent Hybrid pulmonary valve replacement (hPVR) at The Cleveland Clinic over a 16 month period. A retrospective analysis of all the patients who underwent hPVR at The Cleveland Clinic between August 2019 and December 2020. A total of 11 patients were eligible for hPVR. Two patients required conversion into surgical pulmonary valve replacement, one with significant mediastinal adhesions and the other because of malfunctioning of the delivery system. A total of 6 males and 3 females underwent successful hPVR. Median age was 17 years (range $7-57$ years). The primary diagnoses were congenital pulmonary valve stenosis in 6 patients (native RVOT in 5 patients and 1 patient had a bio prosthetic valve) and Tetralogy of Fallot in 3 patients (native RVOT in 2 patients and 1 patient had an RV to PA conduit). All patients had moderate to severe pulmonary regurgitation and only one patient had significant bio-prosthetic valve stenosis. 6 patients were asymptomatic. Three patients had symptoms of dyspnea on exertion (NYHA functional class II-IV). Pulmonary regurgitation decreased to only trivial to none in 7 patients and mild regurgitation in 2 patients with resolution of symptoms in all. The average peak/mean pressure gradient was $13 \mathrm{mmHg} / 6.6 \mathrm{mmHg}$ immediately post procedure. Short term follow up showed excellent valve function with the average peak and mean valve gradient of $14 \mathrm{mmHg}$ and $7 \mathrm{mmHg}$, respectively. There was no significant pulmonary valve regurgitation and no recurrence of symptoms. Procedural complications included a small contained conduit tear in 1 patient and in another, a small contained MPA tear with a small flail segment of tricuspid valve was noted. The average length of ICU stay was 1.3 days (range 1-2 days) and average total hospital stay was 4 days (range 2-11 days). Hybrid PVR is emerging as a promising technique for PVR in high risk patients that are not eligible for current trans-catheter pulmonary valve replacement. In our small cohort, we saw excellent short term results after $\mathrm{hPVR}$.

ETHICS DECLARATIONS

Conflict of interest: The authors declare that they do not have any conflict of interests.
Ethical Approval: Not applicable.

Consent for Publication: Not applicable.

\section{Intravenous alternative to inhalational Nitric Oxide in resource constrained environment: Randomized Controlled Trial}

\section{Neeraj Awasthy, Gaurav Kumar}

Max Hospital, Delhi, India

In children with congenital heart disease with Pulmonary artery Hypertension, inhaled Nitric Oxide has been advised to use for Acute vasoreactivity testing as Class I recommendation. Inhaled Nitric Oxide has some disadvantages with some patients failing to respond to Nitric Oxide inhalation. Its use as an acute vasodilator is cumbersome, requiring an expensive medical form of Nitric Oxide gas and involving a complicated delivery system and monitoring equipment. We hypothesized that Intravenous Sildenafil is equally effective and accurate for Acute Vaosreactivity testing with no significant difference as compared to Nitric Oxide in subgroup of children with congenital heart disease with PAH. Mann Whitney test was used to assess association Nitric Oxide and IV Sildenafil for all variables. There was no significant difference between the values of Nitric Oxide vs Sildenafil confirming the assumption that IV sildenafil is equally efficacious for Pulmonary Vasoreactivity testing when compared to Nitric Oxide.

\section{ETHICS DECLARATIONS}

Conflict of interest: The authors declare that they do not have any conflict of interests.

Ethical Approval: Not applicable.

Consent for Publication: Not applicable.

\section{Percutaneous Coronary Intervention with Everolimus-Eluting Versus Sirolimus-Eluting Stents in Diabetic Patients Presented with Acute Coronary Syndromes: A Short-Term Comparative Clinical Outcome Study}

Karim Mohamed Fahmy, Mohamed Ibrahim², Raghda Ghonimy El Sheikh ${ }^{1}$, Mai Abdulmonaem

Salama ${ }^{1}$, Medhat Mohamed Ashmawy ${ }^{1}$, Hatem Mohamed ElSokkary ${ }^{1}$

${ }^{1}$ Department of Cardiology, Faculty of Medicine, Tanta University, Egypt. ${ }^{2}$ Department of Cardiology, Faculty of Medicine, Helwan University, Egypt

The use of drug-eluting stents (DES) resulted in innovative progress in the field of interventional Cardiology. Nowadays, the most common indication for coronary stenting is acute coronary syndromes (ACS) due to the better clinical consequences of PCI compared with conservative management. However, in diabetic patients, there is a large debate in the literature regarding the selection of an optimal drug-eluting stent. This work aimed to compare short term clinical outcome post-Percutaneous Coronary intervention with Everolimuseluting stents (EES) versus Sirolimus-eluting stents (SES) in diabetic patients presented with acute coronary syndromes. The present study was carried out on 120 diabetic patients presented with acute coronary syndromes (Non-STEMI, Unstable angina) and divided into two groups; group 1 included patients treated with PCI with Everolimus eluting stenting (EES), and group 2 included patients treated with PCI 
with Sirolimus-eluting stenting (SES). After 6 months of follow-up, the percentages of treated patients who were complicated with sudden cardiac death, congestive heart failure, and planned further PCI were $5 \%, 6.7 \%$, and $6.7 \%$, respectively in group 1 , and were $3.3 \%, 8.3 \%$, and $10 \%$ respectively in group 2 . While no acute coronary syndrome or repeated coronary angiography was reported in both groups. In the current study, there was no statistically significant difference between the EES group and the SES group in diabetic patients either during implantation or during 6 months follow up (P-value $>0.05)$. In this current study, both EES and SES are comparable to each other regarding the treatment of acute coronary syndrome in diabetic patients. Abbreviations: PCI: Percutaneous Coronary Intervention, DES: drug-eluting stenting, EES: Everolimus eluting stenting, SES: Sirolimus-eluting stenting, PES: paclitaxel-eluting stents LL: In-stent late loss, BMS: Bare-metal stents, CABG: Coronary artery bypass grafting, ACS: Acute coronary syndrome, MACE: Major adverse cardiac effect. Keywords: Everolimus eluting stenting, Sirolimuseluting stenting, diabetic patients.

\section{ETHICS DECLARATIONS}

Conflict of interest: The authors declare that they do not have any conflict of interests.

Ethical Approval: Not applicable.

Consent for Publication: Not applicable.

\section{An Attempt to Overcome the Size Limitation of Pulmonary Percutaneous Valve Implantation in Pre- stented native Right Ventricular Outflow Tract: First Experience with Myval Transcatheter Heart Valve in Large RVOT}

\section{Alejandro Rodríguez Ogando ${ }^{1}$, Fernando Ballesteros Tejerizo², Jose Luis Zunzunegui Martinez ${ }^{1}$ \\ ${ }^{1}$ Gregorio Marañon Hospital, Madrid, Spain. ${ }^{2}$ Gregoeio Marañon Hospital, Madrid, Spain}

Pulmonary percutaneous valve implantation (PPVI) is feasible with satisfactory mid-term results in patients with native right ventricular outflow tract (RVOT) and has been increasingly used instead of surgically implantable pulmonary valves. Creating a stable landing zone with a diameter less than the largest commercially available valve (previously available $29 \mathrm{~mm}$ and currently available $32 \mathrm{~mm}$ ) is crucial for technical success of the procedure, limiting the number of suitable candidates for PPVI. The aim of this study is to present our results and feasibility of PPVI with Myval transcatheter heart valve in a large native RVOT (pre-stented) lesion who had Tetralogy of Fallot surgically corrected. Four tetralogy of Fallot patients were enrolled. Before PPVI, severe pulmonary regurgitation (mean pulmonary regurgitation fraction, $43.4 \% \pm 5.2 \%$; range, $38.2-48.6 \%)$ and enlarged right ventricular volume (mean indexed right ventricular end-diastolic volume, $153.7 \pm 10.3 \mathrm{ml} / \mathrm{m}^{2}$; range $143.4-164 \mathrm{ml} / \mathrm{m}^{2}$ ) were present. The median age at PPVI was 17.2 years (range 11-20 years). All patients were pre-stented with Andra-stent XXL, mounted on Nucleus Balloon of $28 \mathrm{~mm}$ (Numed), Andra Balloon of $32 \mathrm{~mm}$ (2 patients) and Valver Balloon of $35 \mathrm{~mm}$ (Balton). Three months after pre-stenting, PPVI were successfully implanted with Myval THV of $26 \mathrm{~mm}$ (first case) and Myval THV XL of $32 \mathrm{~mm}$, loaded on the 26 Fr GORE $\AA$ DrySeal sheath. Myval THV crimped on Navigator delivery system was inserted in the target RVOT area and positioned carefully. In the last case ( $35 \mathrm{~mm}$ RVOT diameter), the valve was deployed at the target area by adding $8 \mathrm{ml}$ extra-volume for the nominal balloon configuration $(40 \mathrm{ml})$ to reach $35 \mathrm{~mm}$. Pulmonary artery angiogram showed no significant pulmonary regurgitation and three-dimensional transesophageal echocardiography showed good valve coaptation. No significant periprocedural complications were noted in any patient. There was no adverse event associated with the valve. Our results demonstrate the first experience of the feasibility of successfully implanting Myval THV in large native RVOT and the first experience with a $32 \mathrm{~mm}$ BE-THV in a patient with a large pre-stented native RVOT lesion (up to $35 \mathrm{~mm}$ ) without any procedural complications. The upcoming new device technology (valves and delivery systems) could facilitate and extend PPVI in conduit-free "native" RVOT. Future studies with a larger number of patients can further establish the safety and effectiveness of this novel BE-THV for the treatment of large native RVOT lesions.

ETHICS DECLARATIONS

Conflict of interest: The authors declare that they do not have any conflict of interests.

Ethical Approval: Not applicable.

Consent for Publication: Not applicable.

\section{Effect of Previous Percutaneous Coronary Intervention on Early Outcome After Coronary Artery Bypass Grafting}

\section{Yasser Mubarak}

Minia University, Minia, Egypt. Madinah Cardiac Center, Madinah Al Munawarra, Saudi Arabia

Previous percutaneous coronary intervention (PCI) may affect or alter the outcome in patients who undergo coronary artery bypass grafting (CABG). To evaluate and compare early in-hospital postoperative outcomes in patients underwent CABG with or without previous PCI. The study included 160 patients underwent isolated elective on-pump CABG at our department from January 2010 to December 2014. Patients with previous PCI $(\mathrm{n}=38)$ were compared to patients without previous PCI $(n=122)$. Preoperative, operative and early inhospital postoperative data were analyzed. The study end points were in-hospital mortality and postoperative major adverse events. There was insignificant difference between both groups regarding preoperative demographic data, risk factors, left ventricular ejection fraction, NYHA class, Euroscore, presence of left main disease, reoperation for bleeding, postoperative acute myocardial infarction, neurological deficit, need for renal dialysis, hospital stay and in-hospital mortality. The average time from PCI to CABG was $13.9 \pm 5.4$ years. Group of previous PCI had significant increase in proportion of patients with any in-hospital major adverse event $(15.8 \%$ versus $2.5 \%, \mathrm{p}=0.002)$. On multivariate analysis, only previous PCI was a significant predictor of major adverse events (OR: 0.16, 95\% CI: 0.03-0.71, $\mathrm{p}=0.01$ ). Previous percutaneous coronary intervention has a significant effect on the incidence of early major adverse events after $\mathrm{CABG}$, but does not affect the mortality rate. Further large-scale and long-term studies are recommended.

\section{ETHICS DECLARATIONS}

Conflict of interest: The authors declare that they do not have any conflict of interests.

Ethical Approval: Not applicable.

Consent for Publication: Not applicable. 
061. Multi-center Experience: Early Outcome of Mitral Valve Repair in Patients with Ischemic Mitral Regurgitation

\section{Yasser Mubarak}

Minia University, Minia, Egypt. Madinah Cardiac Surgery, Madinah Al Munawarra, Saudi Arabia

Ischemic mitral regurgitation (IMR) is associated with poor outcomes. The optimal surgical strategy for management of IMR is still debated. The objective of this study is to evaluate early mortality and morbidity of mitral valve repair ( $\mathrm{MVr}$ ) in patients with IMR undergoing coronary artery bypass grafting (CABG). We performed a retrospective, observational; cohort study was undertaken of prospectively collected data on 136 consecutive CABG patients with IMR undergoing MVr between January 2016 and January 2020. Perioperative echo findings, operative procedure, and outcome were analyzed. The overall mortality rate was $4.4 \%$. Mitral valve repair with low Ejection Fraction (EF) has a fourfold increase in the risk of death compared with $\mathrm{MVr}$ with preserved $\mathrm{EF}>30 \%$. However, after adjusting for preoperative risk factors, the number of grafts was not an independent risk factor for mortality (OR 0.18 , 95\% CI $0.03-2.81$, $\mathrm{p}=0.84$ ). Multivariable analysis showed that preoperative LVEF (OR 1.14, 95\% CI 0.82-4.86, $\mathrm{p}=<0.01$ ), and preoperative LVESD (OR 1.03, 95\% CI 0.65-3.51, $\mathrm{p}=<0.01$ ) and preoperative LVED (OR 0.99, 95\% CI 0.64-3.28, $\mathrm{p}=0.04$ ) were independent risk factors of mortality. MV Repair can be performed safely, concomitantly with CABG, in patients with (moderate, moderately severe, and severe) IMR.

\section{ETHICS DECLARATIONS}

Conflict of interest: The authors declare that they do not have any conflict of interests.

Ethical Approval: Not applicable.

Consent for Publication: Not applicable.

\section{Fetal Pulmonary Valvuloplasty: Single Center Experience}

Victorio Lucini, Grinenco Sofia, Lucas Otaño, Marantz Pablo, Horacio Aiello, Luis Trentacoste, Marcelo Rivarola

Hospital Italiano, Buenos Aires, Argentina

A group of patients with pulmonary atresia (PA) or critical pulmonary stenosis (CPS) and with intact ventricular septum evolve prenatally with hypoplasia of the right ventricle (RV) and univentricular circulation at birth, with increased mid and long-term associated mortality. Fetal pulmonary valvuloplasty (FVP) has been proposed as a means to promote RV growth and avoid significant RV hypoplasia at birth. The aim of this study is to present a series of cases of patients selected for FPV at Hospital Italiano de Buenos Aires. Patients with membranous PA or CPS; intact ventricular septum and hypoplastic borderline RV detected prenatally; with no significant maternal nor fetal extracardiac morbidity, and selected for FPV in our center between February 2012 and February 2021 were included. Echocardiographic data, procedural aspects and clinical outcome were assessed retrospectively. Among 9 patients selected for FPV, 1 mother declined the procedure and in 3 patients FPV could not be performed because appropriate fetal position could not be achieved, among these; 2 evolved with a RV hypoplasia and univentricular physiology; one was lost to follow-up and 1 evolved with a biventricular circulation after postnatal pulmonary valvuloplasty. From the 5 patients that underwent FPV, one fetus with PA died immediately during FPV, and 4 fetuses with CPS underwent successful FPV with a favorable clinical outcome, and postnatal biventricular circulation after neonatal pulmonary valvuloplasty. There were no maternal complications. Fetal pulmonary valvuloplasty is a feasible procedure. All fetuses with successful CPS have postnatal biventricular circulation in our series.Unfavorable fetal position and atresia of the pulmonary valve posed technical difficulties. Large multicenter studies are needed to assess patient selection criteria and clinical outcome.

ETHICS DECLARATIONS

Conflict of interest: The authors declare that they do not have any conflict of interests.

Ethical Approval: Not applicable.

Consent for Publication: Not applicable.

\section{Transcatheter Fontan Fenestration from LPA to $\mathbf{L A}$}

Ahmad Khalil ${ }^{1,2}$, Josue Diaz-Frias ${ }^{1,2}$, Christopher Ratnasamy ${ }^{1,2}$, Joseph Vettukattil ${ }^{1,2}$

${ }^{1}$ Helen DeVos Children's Hospital, Grand Rapids, USA. ${ }^{2}$ Michigan State University, Grand Rapid, USA

In Fontan patients, a fenestration is frequently created between the Fontan baffle and the right atrium in an effort to delay the potential complications of resultant a high venous pressure including pleural effusions, plastic bronchitis and protein-losing enteropathy. Also, a Fontan fenestration is frequently used as an access point for catheter ablation of arrythmias. In many patients, a fenestration creation might be indicated, whether for a failing non-fenestrated Fontan, or after spontaneous closure of the fenestration. Transcatheter approach is preferred given the higher morbidity with the surgical approach, especially with the extra-cardiac modification where a Gore-Tex tube is placed between the inferior vena cava and the pulmonary arteries. But, because of the angle needed for the puncture from the femoral or internal jugular access, the Gore-Tex conduit having a smooth inner surface that is difficult to engage with the needle, and the conduit being tough, traditional method of puncturing through the conduit might be difficult and a nontraditional method is sometimes needed. A 25-year-old lady with history of Tricuspid Valve Atresia underwent modified lateral tunnel Fontan procedure in 1996. She developed recurrent atrial arrhythmia with a dilated Fontan circuit, then underwent Fontan revision to a fenestrated extracardiac Fontan conduit with $22 \mathrm{~mm}$ Gore-Tex tube and a $5 \mathrm{~mm}$ fenestration in 2020, along with placement of dual chamber epicardial pacemaker and Maze procedure. Nevertheless, she continued to have significant arrhythmia burden. Her Fontan fenestration could not be visualized by either TEE or angiogram. Cardiac CT was performed and showed that the GoreTex Fontan conduit extends very low, with no possible area of adjacency between the IVC and the RA (Fig. 1), making her unsuitable for fenestration creation in the classical position given the challenges discussed above. An interactive 3D visualization, based on the Cardiac CT, showed that the left branch PA was in close proximity to the roof of the LA (Fig. 2). With access through the right IJ vein, angiogram showed the LPA/LA junction at the roof of the left atrium. Under fluoroscopic guidance and transesophageal echocardiogram, 8.5 French Agilis sheath was introduced into the area, and the hard end of a Mailman guidewire was used to puncture the LPA and to enter the LA (Fig. 3). A $4 \times 12 \mathrm{~mm}$ then a $4 \times 20 \mathrm{~mm}$ Sprinter OTW high pressure balloons were advanced consecutively and inflated with the balloon communicating across the LPA and the roof of LA (Fig. 4). Another wire was advanced and the Agilis sheath was advanced further into the LA over the two wires, giving access for EP study. EP study was carried out and arrhythmogenic focus was 


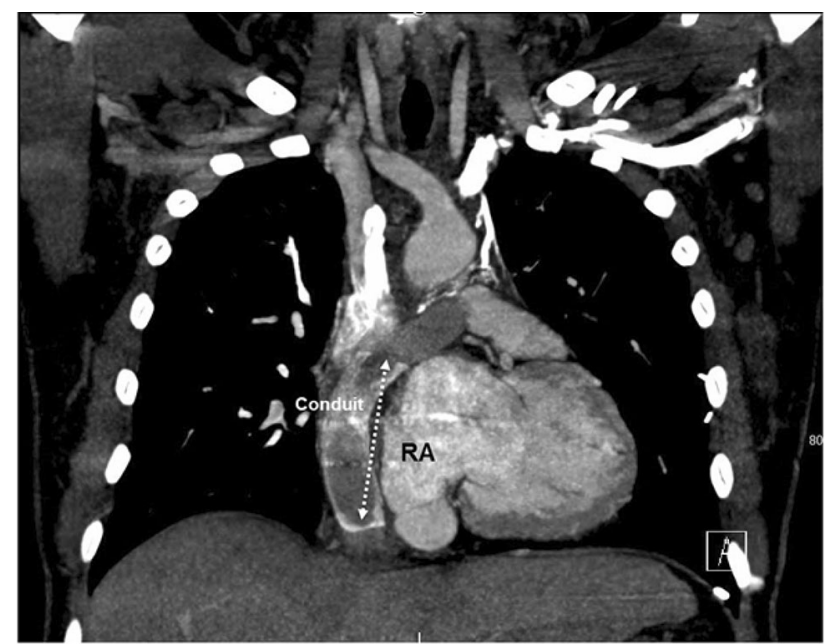

Fig. 1 Cardiac CT showing that Gore-Tex Fontan conduit extends very low, with no possible area of adjacency between the native IVC and the RA

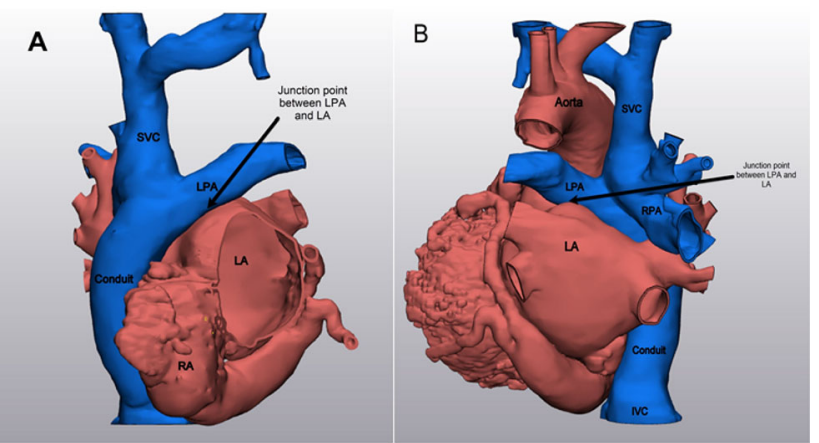

Fig. 2 Interactive 3D visualization images based on the cardiac CT, showing inferior surface of LPA on top of the LA roof in anterior view $(\mathrm{A})$ and posterior view $(\mathrm{B})$

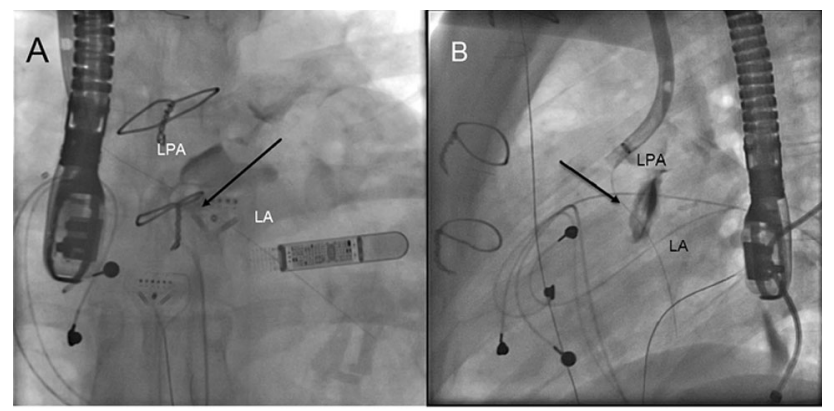

Fig. 3 Fluoroscopic images showing wire position crossing from LPA to LA on AP projection (A) and RAO projection (B)

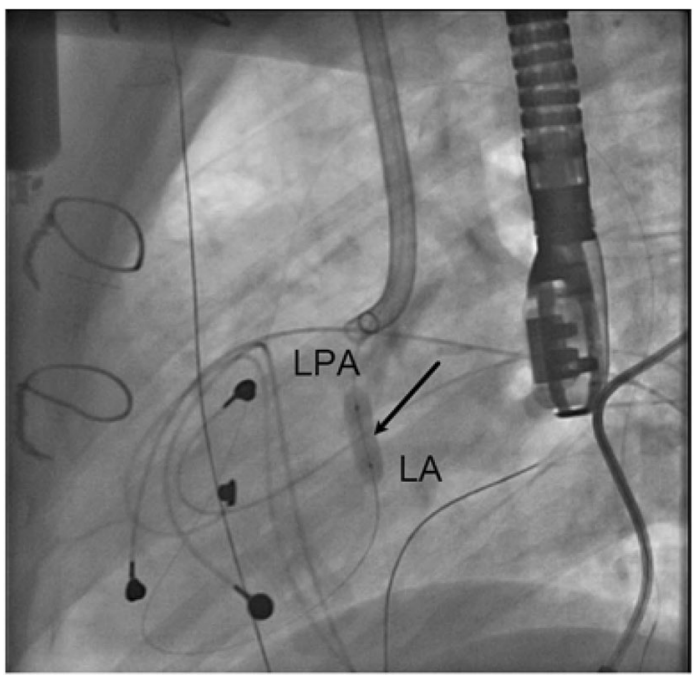

Fig. 4 RAO fluoroscopic images showing balloon positioned over the wire across the communication between LPA and LA

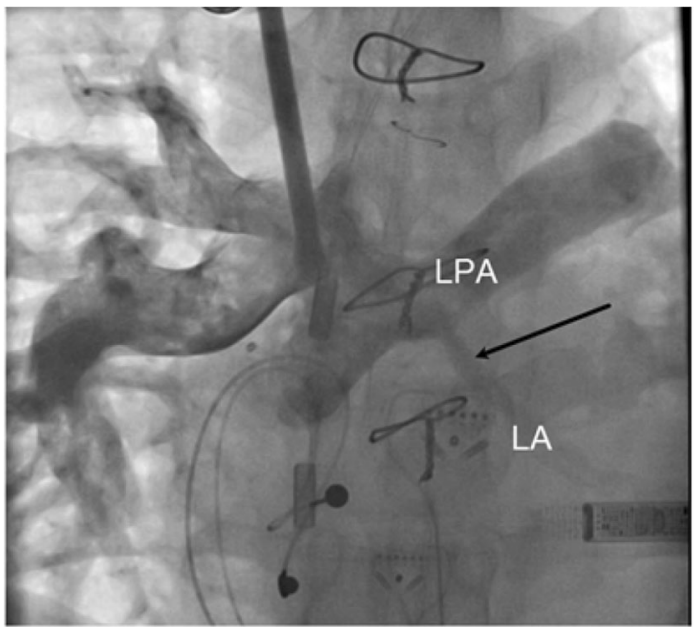

Fig. 5 AP angiogram through the right IJ vein into the MPA showing contrast draining into the LA through the patient fenestration between LPA and LA

successfully ablated. At the end of the study and after removal of all catheters, an angiogram of the LPA was performed and showed patent fenestration from LPA to LA, with good flow and without extravasation into the pericardial space (Fig. 5). Creation of Fontan fenestration can be done safely using transcatheter approach, avoiding the morbidity the accompanies surgical procedures, although nontraditional approach might be needed in some cases.

\section{ETHICS DECLARATIONS}

Conflict of interest: The authors declare that they do not have any conflict of interests.

Ethical Approval: Not applicable.

Consent for Publication: Not applicable. 


\section{Polypharmacy Overdose and Increased Percutaneous ECMO Utilization at a Tertiary Care Center During the COVID-19 Pandemic}

\author{
Barry O'Callaghan, Gareth Morgan, Shannon Buckvold, Jenny \\ Zablah
}

Children's Hospital Colorado, Aurora, CO, USA

Background: The COVID-19 pandemic has placed significant strain on both individuals and health services. We report a cluster of adolescent patients with polypharmacy overdose refractory to medical therapy and cannulated to percutaneous femoral veno-arterial (VA) extracorporeal membrane oxygenation (ECMO) during the early phase of the COVID-19 pandemic at our tertiary care center. ECMO is an effective management strategy for the provision of cardiorespiratory support in patients with potentially reversible, lifethreatening conditions that are unresponsive to conventional therapy. Our institutional ECMO database was interrogated for patients requiring ECMO for vasoplegic shock secondary to para-suicidal events. Patient demographics, clinical data, procedural data and outcomes were assessed retrospectively to profile this subgroup of patients who required ECMO support. Four pediatric patients received percutaneous femoral ECMO cannulation and a single patient received surgical ECMO cannulation during this period for similar indications. Herein we describe our procedural approach to percutaneous ECMO in children $(>30 \mathrm{~kg})$ and the outcomes of those who underwent ECMO cannulation during the pandemic timeframe. Three patients who had percutaneous ECMO survived and one died secondary to complications of polypharmacy overdose. A single patient who was not suitable for percutaneous ECMO cannulation was surgically cannulated to ECMO and died thereafter. A cluster of adolescent patients with polypharmacy ingestion during the early stage of the COVID-19 pandemic resulted in a temporal increase in percutaneous ECMO utilization at our institution. Percutaneous ECMO is a potentially life-saving procedure for the stabilization of critically-ill pediatric patients and remains an effective modality to improve survival to discharge.

ETHICS DECLARATIONS

Conflict of interest: The authors declare that they do not have any conflict of interests.

Ethical Approval: Ethical Approval to report this case series was obtained from our Institutional Review Board.

Consent for Publication: Not applicable.

\section{Left Ventricular Dilation is Associated with Decreased Left Ventricular Systolic Function After Transcatheter PDA Closure in Premature Neonates}

\section{Aravinth Karunanandaa MD ${ }^{1}$, Kamala Swayampakala PhD, MSPH $^{2}$, Joseph Paolillo MD ${ }^{1,3}$, Matthew Saxonhouse MD ${ }^{1}$, Matthew C. Schwartz MD M, $^{1,3}$ \\ ${ }^{1}$ Atrium Health Levine Children's Hospital, Charlotte, USA. ${ }^{2}$ Atrium Health Carolinas Medical Center, Charlotte, USA. ${ }^{3}$ Sanger Heart and Vascular Institute, Charlotte, USA}

Transcatheter patent ductus arteriosus (PDA) closure has emerged as an important therapeutic option for premature neonates. Following PDA closure, left ventricular (LV) systolic function can decrease due to an acute increase in afterload. Changes in LV systolic function have not been well described in premature neonates after device PDA closure. Likewise, patient factors that are associated with the development of LV systolic dysfunction after device closure have not been described in this population. We retrospectively identified all premature neonates less than $3 \mathrm{~kg}$ who underwent successful transcatheter PDA closure at our center between January 1, 2015 through January 31, 2021. Patients with decreased LV systolic function prior to the procedure were excluded. Relevant preprocedural and procedural patient variables were extracted and echocardiogram reports after catheterization were reviewed. Changes in LV systolic function after closure were described and analysis performed to identify patient variables that were associated with the development of decreased LV systolic function (defined as LV ejection fraction $(\mathrm{EF}) \leq 55 \%$ ) at $24 \mathrm{~h}$ after PDA closure. 23 patients were included with a with a mean gestational age of $25.8 \pm 2.3 \mathrm{wks}$, a mean age at catheterization of $65 \pm 25.7$ days, and a mean procedural weight of $1894 \pm 622$ g. $13(56.5 \%)$ patients were on ventilator support at the time of catheterization. All had an LVEF $>55 \%$ prior to catheterization with a mean LVEF of $68 \pm 7.5 \%$. The mean LV end-diastolic dimension $\mathrm{z}$-score was $+3.1 \pm 1.2$ and $22(96 \%)$ patients had a moderate or greater PDA. All patients had successful closure; $18(78.3 \%)$ had no residual flow and $5(21.7 \%)$ had trivial residual flow by echocardiogram at the end of the case. On follow up echocardiogram at $24 \mathrm{~h}$ after PDA closure, 6 (26\%) had an LVEF $<55 \%$ with a mean LVEF of $48 \pm 7.2 \%$ and LVSF of $22 \pm 3.8 \%$. Patients who had an LVEF $\leq 55 \%$ at $24 \mathrm{~h}$ had a higher pre-procedure LV end-diastolic dimension z-score (4.2 vs 2.7 , $\mathrm{p}=0.004)$ and a higher preprocedure LV end-diastolic volume (17.6 vs 11.3, $\mathrm{p}=0.03)$ compared to those who demonstrated an LVEF $>55 \%$ at $24 \mathrm{~h}$ after PDA closure. Of the 6 patients who had $\mathrm{LVEF} \leq 55 \%$ at $24 \mathrm{~h}$ after the procedure, none were placed on inotropic support and all were discharged from the hospital. One patient did not have further imaging in our system, but the remaining 5 patients all showed normalization of LVEF $(>55 \%)$ at a median of 30 (range 6-67) days post catheterization. In a cohort of premature neonates who underwent transcatheter device PDA closure, the development of decreased LV systolic function $24 \mathrm{~h}$ after closure was common, but normalized in all patients at short-term follow up. Patients with LV dilation prior to the procedure were at increased risk for the development of abnormal LV systolic function after closure.

ETHICS DECLARATIONS

Conflict of interest: The authors declare that they do not have any conflict of interests.

Ethical Approval: Not applicable.

Consent for Publication: Not applicable.

\section{Novel Diabolo Modification of a Large Diameter Stent to Treat Cyanosis Related to Microvascular Pulmonary Arteriovenous Malformations}

\author{
$\underline{\text { Sarosh Batlivala }}^{1,2}$, Amr Matoq ${ }^{1}$ \\ ${ }^{1}$ Cincinnati Children's Hospital, Cincinnati, USA. ${ }^{2}$ University \\ of Cincinnati College of Medicine, Cincinnati, USA
}

A large burden of microvascular pulmonary arteriovenous malformations (pAVMs) can lead to profound hypoxemia. An as yet unidentified "hepatic factor" is postulated to play a mechanistic role in the development of these lesions. Certain subsets of patients with congenital heart disease, particularly those with heterotaxy syndromes and after complex Fontan palliation, are at particular risk to develop pAVMs. Ideally, the underlying cause leading to pAVM development is identified and corrected early, though in some patients with longstanding pAVMs, adjusting flow to optimize the circulation (i.e. 
distribution of the putative "hepatic factor") may not lead to rapid resolution of the pAVMs. We report the case of a teenage girl with heterotaxy syndrome $\mathrm{s} / \mathrm{p}$ Fontan who had significant pAVMs, undergone Fontan revision that resulted in equal distribution of hepatic venous effluent to both lungs, but who remained profoundly desaturated, polycythemic, and with very limited exertional tolerance for more than one year after Fontan revision. Rather than perform the drastic action of complete pulmonary arterial occlusion/ligation or pneumonectomy, we performed a novel method to produce a diabolo modification of a large covered stent to significantly reduce flow to the affected lung, while maintaining the potential to serially dilate the stent if the pAVMs regress over time. This technique involved placement of a suture around the central portion of a long CP covered stent to create the diabolo configuration. The procedure resulted in an immediate increase in systemic saturations while maintaining some flow patency for future PA rehabilitation if indicated. Diabolo stent configuration can provide unique therapeutic options for specific situations. Though other techniques have been described in congenital cardiac catheterization, we report another technique for potential use with larger stents and vessel caliber.

\section{ETHICS DECLARATIONS}

Conflict of interest: The authors declare that they do not have any conflict of interests.

Ethical Approval: Not applicable.

Consent for Publication: Not applicable.

\section{Piccolo Device Utility in the Treatment of Pulmonary Sequestration in Infants}

\section{Justo Santiago, Jorgue Alvarado, Claudia Flores, Helena Franco}

\section{Colombia Cardiovascular Foundation, Floridablanca, Colombia}

Pulmonary sequestration is a rare anomaly that represents 0.15 to $6.4 \%$ of all pulmonary malformations (1). There is currently evidence supporting endovascular embolization as a definitive treatment option (2). The most widely used devices have been coils. We present a series of patients under $3.5 \mathrm{~kg}$ who were approached using the Amplatzer Piccolo devices. Three patients under three months of age (Table Table 1 Hemodynamic and angiographic data analyses comparing Gore-Tex valved and Homograft conduits; reported as median (IQR)TotalGore-TexHomograftp-valueN $=56 \mathrm{~N}=18 \mathrm{~N}=39$ Conduit diameter $(\mathrm{mm})$ Nominal20 $(16,23) 16(16,20) 22(18,24) 0.005$ Pre- intervention12 $(10,15.8) 10.9(9.4,12.9) 13(10.2,16.5) 0.02$ Post-intervention19.2 (18.4, 21)18.6 (17.5, 20)19.9 (18.5, 22)0.16Percent change in conduit diameter (\%)Nominal to pre-intervention64.1 $(52.6,77.5) 63.2(55.6,72.7) 64.1(49.6,80) 0.95$ Pre- to post-intervention157.4 (127.9, 183.5)169.7 (151.2, 188.7)151.7 (118.6, 183.5)0.07Nominal to post-intervention100 (12, 110.5)107.5 (100, 118.75)100 (16.9, 109.1)0.03Conduit peak gradient (mmHg)Pre-intervention42 $(33,47) 39(29,43) 42(33,49) 0.37$ Post-intervention 13 $(9,17) 12(6,16) 14(10,19) 0.12$ Percent Change in Conduit Gradient (\%)32.2 (23.1, 44.5)30.3 (26.2, 38.1)33.4 (22, 53.9)0.481) with clinical manifestations of pulmonary overcirculation, prior informed consent of the parents, underwent cardiac catheterization and endovascular occlusion of the aberrant vessels that nourished the pulmonary sequestration using the main implant of Amplatzer devices Piccolo through 4 frech release system.

In all cases, the implant could be performed without complications, they evolved favorably with clinical and radiological improvement and remission of the pulmonary hyperflow data. In one patient, residual flow was appreciated that warranted the implantation of an additional coil with total closure of the flow (Figs. 1 and 2).

The complications of pulmonary sequestration are the development of heart failure and recurrent pneumonia. The endovascular treatment of this pathology was described for the first time by Rothman (5). Since then, the most widely used devices have been coils; however, in few cases of large children and adults the use of Amplatzer-type devices has been described $(3,6-8)$. In these patients, depending on the diameter of the device, the use is warranted of 5 or 6 French release systems. The Piccolo ductus arteriosus occluder device is a self-expanding device made of wire mesh assembled with nitinol and interwoven polyester for remote tissue growth promotion (9). It has a central waist from 3 to $5 \mathrm{~mm}$ in diameter and two retention discs

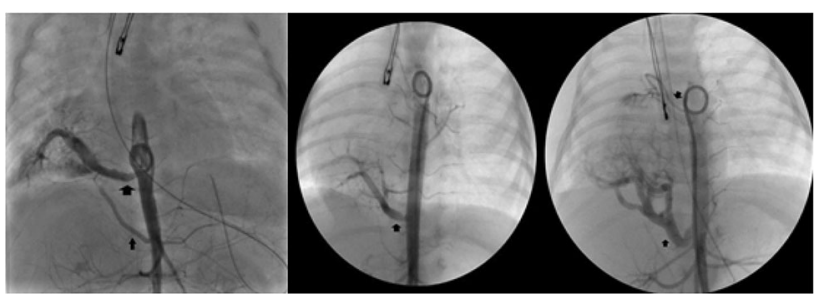

Fig. 1 Basal aortogram showing aberrant vessels

Table 1 Characteristics of patients with pulmonary sequestration who underwent endovascular occlusion with Amplatzer Piccolo devices

\begin{tabular}{|c|c|c|c|}
\hline & Case 1 & Case 2 & Case 3 \\
\hline Age (days) & 60 & 24 & 14 \\
\hline Gender & Female & Female & Male \\
\hline Peso: (kg rs) & 3.46 & 2.7 & 3.3 \\
\hline Associated Heart Disease & VSD, PPVAD (Scimitar), SCDV, ASD, & PPVAD (Scimitar), ASD & ASD \\
\hline Clinical manifestation & $\begin{array}{l}\text { Heart failure, Enterocolitis III-B Heart } \\
\text { failure }\end{array}$ & Heart failure, Enterocolitis & $\begin{array}{l}\text { Intercostal } \\
\text { pulling }\end{array}$ \\
\hline Secuestro type & Extraloba & Extralobar & Intralobar \\
\hline Aberrant vessel number/diameter & $2 / 3.9 \mathrm{~mm}, 1.6 \mathrm{~mm}$ & $1 / 3.9 \mathrm{~mm}$ & $1 / 3 \mathrm{~mm}$ \\
\hline $\begin{array}{l}\text { Fluoroscopy time (min)/radiation (mGy } \\
\mathrm{cm}^{2} \text { ) }\end{array}$ & $31.48 / 5036.36$ & $21.17 / 1268.42$ & $8.4 / 335.77$ \\
\hline Devices used & $\begin{array}{l}\text { Piccolo 5-6 mm } \\
\text { Coil 3-10 mm }\end{array}$ & $\begin{array}{l}\text { Piccolo 5-4 mm, Piccolo } 4-4 \mathrm{~mm} \text {, Coil } \\
\text { 5-10 mm }\end{array}$ & $\begin{array}{l}\text { Piccolo } \\
\quad 5-6 \mathrm{~mm}\end{array}$ \\
\hline
\end{tabular}




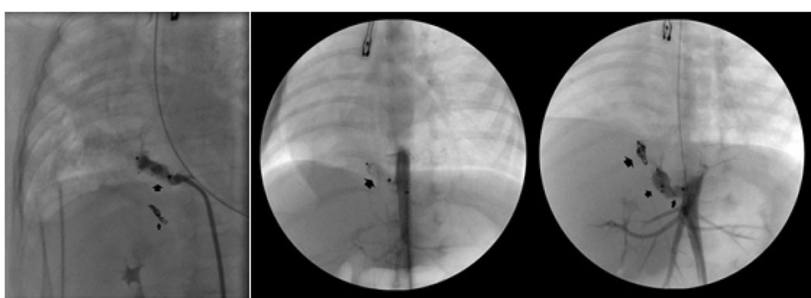

Fig. 2 Post-implant angiography of the Amplatzer Piccolo devices

with a diameter from 4 to $6.5 \mathrm{~mm}$, uses a $4 \mathrm{~F}$ release system which is an advantage for use through the artery of young children like those described in our series. The endovascular treatment of pulmonary sequestration by means of the implantation of Piccolo Amplatzer devices is a feasible and safe technique that can be used in infants and newborns.

Bib*liography

1. Rodríguez $\mathrm{L}$ et al. Tratamiento endovascular del secuestro pulmonar con dispositivos tipo Amplatzer ${ }^{\circledR}$. An de pediat 2012: 76; 285-289).

2. Brown $\mathrm{S}$, et al. Treatment strategies for pulmonary sequestration in childhood: resection, embolization, observation? Acta Cardiologica, 67:6, 629-634.

3. Rothman A, Tong AD. Percutaneous coil embolisation of superfluous vascular connections in patients with congenital heart disease. Am Heart J 1993;126:206-13.

4. Ganeshan A, Freedman J, Hoey ET, Steyn R, Henderson J, Crowe PM. Transcatheter coil embolisation: a novel definitive treatment option for intralobar pulmonary sequestration. Heart Lung Circ 2010;19:561-565.)

5. Schwartz M. The Amplatzer vascular plug and Amplatzer vascular plug II for vascular occlusion procedures in 50 patients with congenital cardiovascular disease. Catheter Cardiovasc Interv 2010 Sep 1;76 (3):411-7.

6. Sathanamdam et al. Amplatzer Piccolo occluder clinical trial for percutaneous closure of the patents ductus arteriosus in patients $\geq 700$ g. Catheter cardiovascular interv. 2020;96:1266-1276.

\section{ETHICS DECLARATIONS}

Conflict of interest: The authors declare that they do not have any conflict of interests.

Ethical Approval: Not applicable.

Consent for Publication: Not applicable.

\section{Safety of Continuing Systemic Anticoagulation Prior to a Cardiac Catheterization in Pediatric Patients: A Los Angeles Center Experience}

\section{Mounica Y. Rao, Patrick M. Sullivan, Cheryl Takao, Sarah Badran, Neil D. Patel \\ Children's Hospital Los Angeles, Los Angeles, USA}

Historically, systemic anticoagulation has been held in adult patients 24-72 h prior to a cardiac catheterization (CC). At our center we typically do not hold anticoagulation prior to CC. In 2020, we first reported on the safety of preforming $\mathrm{CC}$ in 46 pediatric patients while still on systemic anticoagulation. Here we present our findings from a larger cohort of pediatric patients. A single-center retrospective chart review was performed from 11/2014 to $10 / 2019$. Patients who were treated with systemic anticoagulation and undergoing $\mathrm{CC}$ were identified. Individuals on aspirin monotherapy were excluded. Specific variables assessed include sheath size, hemostasis times, interventions performed, indications for anticoagulation, and complications. Data are provided as median [range]. $151 \mathrm{CC}$ were identified in 111 patients who were being treated with systemic anticoagulation. Seven cases were excluded because anticoagulation was held prior to CC. The median age and weight were 6.3 [0.01-20.9] years and 19.3 [2.1-172.5] kg, respectively. The systemic anticoagulation medications used were warfarin $(n=57)$, heparin $(n=52)$, enoxaparin $(n=25)$, fondaparinux $(n=5)$, rivaroxaban $(\mathrm{n}=2)$, and both heparin and warfarin $(\mathrm{n}=3)$. The median precatheterization international normalized ratio in the patients on warfarin was 2.4 [1.4-6.6], and the median pre-catheterization anti-Xa in the patients on heparin was $0.41[0.05-1.96]$. The opening activated clotting time for all cases was 184 [77-308]. The most common indications for anticoagulation were arterial/venous thrombus $(n=45)$, Fontan physiology including primary prophylaxis, fenestration, or thrombus $(\mathrm{n}=32)$, mechanical valve $(\mathrm{n}=27)$, extracorporeal membrane oxygenation $(\mathrm{n}=16)$, heart failure $(\mathrm{n}=8)$, and coronary artery aneurysm $(n=8)$. Ultrasound was utilized for vascular access in 78 cases (54\%). Both arterial and venous access were obtained in 95 cases (66\%), only arterial access in 8 cases $(6 \%)$, and only venous access in 41 cases $(29 \%)$. The largest arterial access obtained was a $7 \mathrm{Fr}$ sheath in 1 case. The most commonly used sheaths for arterial access were a $4 \mathrm{Fr}$ sheath in 50 cases, $5 \mathrm{Fr}$ sheath in 29 cases, 3.3 Fr sheath in 7 cases, and a 3 Fr catheter or 0.018 " dilator in 16 cases. The largest venous access was a $22 \mathrm{Fr}$ sheath, and a sheath larger than $7 \mathrm{Fr}$ was used in 32 cases. Additional anticoagulation was administered during CC in 95 cases (heparin $=90$, bivalirudin $=5$ ). Interventions were performed in 96 cases (67\%): angioplasty $(n=67)$, stent implantation $(n=31)$, vascular embolization $(n=18)$, transeptal puncture $(n=10)$, liver biopsy $(n=10)$, thrombectomy $(n=11)$, fenestration occlusion $(n=6)$, valvuloplasty $(n=2)$, and balloon atrial septostomy $(\mathrm{n}=1)$. The median length of the procedure was 122.5 [15-760] min, and the median time to achieve hemostasis was 18.0 [1-76, IQR: 13-25] min. Complications were present in 6 cases $(4 \%)$ and included sheath dislodgement $(n=1)$, access-site bleeding on either POD \#0 $(\mathrm{n}=1)$ or on POD \#1 ( $=1)$, non-occlusive femoral artery thrombus $(\mathrm{n}=1)$, and a small hematoma $(n=2)$. CC with a variety of interventions and large sheath sizes in pediatric patients on therapeutic anticoagulation can be performed safely. The rate of bleeding related complications is low and observed complications are mild in nature.

ETHICS DECLARATIONS

Conflict of interest: The authors declare that they do not have any conflict of interests.

Ethical Approval: CHLA IRB Approved (CHLA-19-00,456).

Consent for Publication: Not applicable.

\section{Timing of Balloon Atrial Septostomy in Patients with d-TGA and Association with Birth Location and Patient Outcomes}

\section{Courtney Thomas, Sunkyung Yu, Ray Lowery, Jeffrey Zampi}

CS Mott Children's Hospital, Ann Arbor, MI, USA

Patients with d-looped transposition of the great arteries (d-TGA), especially those without an adequate atrial septal defect, can be subject to profound hypoxemia and hemodynamic compromise in the neonatal period. An important component of initial management of these patients is expedited access to medical and interventional therapies such as balloon atrial septostomy (BAS) to avoid prolonged 
hypoxemia. However, a number of patients with d-TGA are delivered at centers without such capabilities. The aim of this study was to evaluate the time from birth to BAS for infants born at a tertiary care center as compared to those requiring transport from other institutions and to examine correlation between time to BAS and patient outcomes. This was a retrospective review of d-TGA patients who underwent urgent or emergent BAS at our institution between January 2010 and January 2021. Our primary outcome was time from birth to BAS. Our secondary outcomes included hospital and ICU length of stay, overall mortality, and evidence of sequelae from neonatal hypoxia or left atrial hypertension including pulmonary hypertension, duration of mechanical ventilation, abnormal neuroimaging, or clinical seizures. Standard descriptive statistics were reported and univariate analysis was performed to examine correlation between time to BAS/birth location and patient outcomes. Of the 96 patients included, $67(70 \%)$ were born at our institution and $69(72 \%)$ were prenatally diagnosed with d-TGA, with only 3 prenatally diagnosed patients born at other institutions. The median time to BAS was $4 \mathrm{~h}$ for patients born at our institution compared to $14.1 \mathrm{~h}$ for those born elsewhere $(\mathrm{p}<0.0001)$. In addition, prior to BAS, patients born at other institutions were more likely to require inotropic support $(\mathrm{p}<0.0001)$ and 2 patients born at other institutions had cardiac arrests prior to BAS. A longer time from birth to BAS was associated with a longer ICU $(r=0.21, \mathrm{p}=0.046)$ and hospital length of stay $(r=0.24, \mathrm{p}=0.02)$ after definitive cardiac surgery as well as an increased likelihood of having an elevated right ventricular (RV) pressure (greater than half systemic) on post-operative discharge echocardiogram $(\mathrm{p}=0.01)$. There were no differences in duration of mechanical ventilation, pulmonary vasodilator requirement, need for ECMO, or mortality between the groups. For patients with d-TGA requiring an urgent or emergent BAS, being born at an outside hospital was associated with significantly longer times from birth to creation of an atrial septal defect. Although overall outcomes were largely similar regardless of place of birth or time to BAS, duration of ICU and hospitalization were longer and elevated RV pressures were more common in those who underwent a later BAS. This may indicate lasting effects of left atrial hypertension and/or post-natal hypoxia in these patients. Delivery of infants with d-TGA at institutions with interventional capabilities may mitigate some of these complications. Further evaluation of late neurologic sequelae could provide additional insight.

\section{ETHICS DECLARATIONS}

Conflict of interest: The authors declare that they do not have any conflict of interests.

Ethical Approval: Not applicable.

Consent for Publication: Not applicable.

\section{Initial Experience with Oversized Balloon Dilation of the exGraft Conduit in the Right Ventricle to Pulmonary Artery Position}

\section{Ryan Halickman, Alexandra Erdmann, Sara Trucco, Melita Viegas, Victor Morell, Jacqueline Kreutzer, Bryan Goldstein \\ UPMC Children's Hospital of Pittsburgh, Pittsburgh, USA}

To describe the early single-center experience with balloon dilation of the expandable exGraft vascular graft in the right ventricle to pulmonary artery (RV-PA) position. Surgical placement of a RV-PA conduit is common in the treatment of congenital heart disease, but conduit longevity can be limited by somatic growth and the development of patient-prosthesis mismatch. The exGraft vascular graft (PECA Labs, Inc. Pittsburgh, PA) is a novel single-layered expanded polytetrafluoroethylene surgical conduit, without an outer wrap, thereby allowing for increased plasticity and low-pressure expansion beyond nominal diameter. These conduits also have radio-opaque markers which allow for visualization of initial conduit size as well as approximating conduit diameter on radiographs (Fig. 1). Use of a dilatable RV-PA conduit could reduce operative frequency and morbidity as well as diminish duration of exposure to conduit dysfunction. Two patients with tetralogy of Fallot, pulmonary atresia, and distal PA stenoses were included in this descriptive series. Surgical conduit placement was performed at 15 days and 3 months of age; nominal conduit diameter was 10 and $8 \mathrm{~mm}$, respectively. Conduit stenosis developed at 8 months of age in both cases, reflecting a

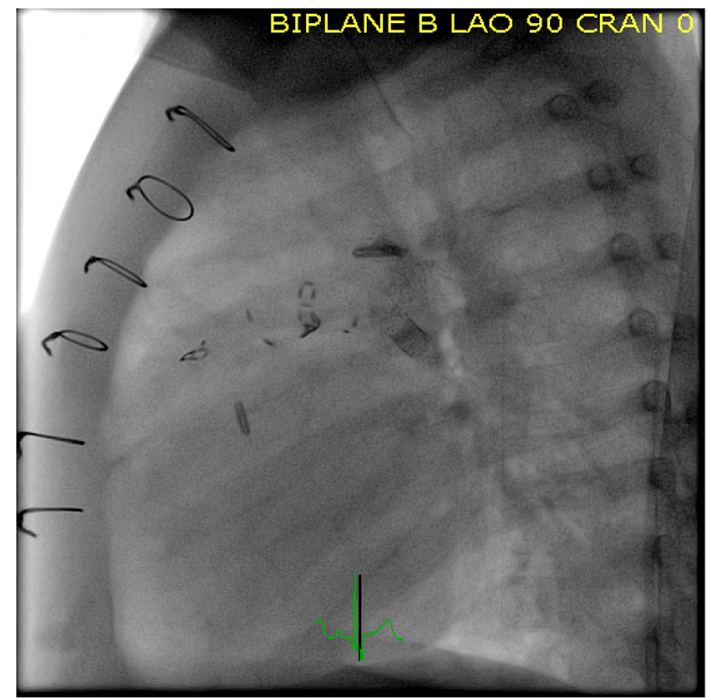

Fig. 1 Lateral projection of exGraft RV-PA conduit highlighting the radio-opaque conduit markers

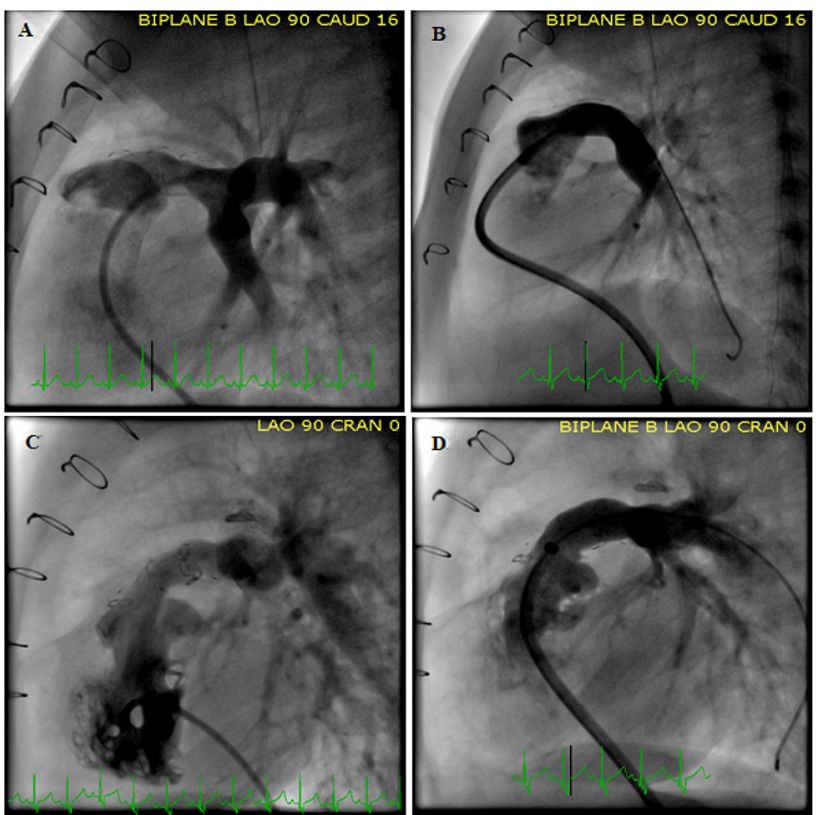

Fig. 2 Lateral angiography of pre- and post-intervention exGraft RVPA conduits in Case 1 (A, B) and case 2 (C, D) 
change in weight of $181 \%$ from implant to time of transcatheter dilation. Hemodynamic assessment revealed a median RV systolic pressure of $70 \mathrm{mmHg}(\mathrm{IQR} 61,78)$ and a median RV:Aortic pressure ratio of $95 \%$ (IQR 80, 109). In both cases, there was evidence of conduit diameter loss, with a median current: nominal conduit diameter ratio of 0.72 (IQR 0.67, 0.76). Balloon angioplasty followed a recommended oversized balloon dilation protocol, with slow dilation up to 8 atmospheres of pressure and serial increase in balloon diameter in $2 \mathrm{~mm}$ increments. Final angioplasty balloon utilized was a mean of $125 \%$ (IQR 113, 138\%) of the nominal conduit diameter, to achieve a final: nominal conduit diameter ratio of 0.99 (IQR 0.94, 1.04). Figure 2 shows angiographic improvement in conduit diameters for both cases. There was associated hemodynamic improvement to a median RV systolic pressure of $51 \mathrm{mmHg}(\mathrm{IQR} 45,56)$ and an RV:Aortic pressure ratio of $61 \%$ (IQR 52, 70). A small, contained conduit injury was identified in one case. No subsequent reinterventions have been performed to date. When utilized in the RV-PA position, the exGraft conduit can be safely and effectively dilated beyond the nominal diameter with resultant improvement in hemodynamic burden. Although recoil occurs following balloon dilation, sustained restoration to nominal conduit diameter occurred in both patients without the need for stent placement. Despite this interventional response, conduit diameter loss following surgical implantation is a concern in this series.

\section{ETHICS DECLARATIONS}

Conflict of interest: The authors declare that they do not have any conflict of interests.

Ethical Approval: Not applicable.

Consent for Publication: Not applicable.

\section{What have We Learnt from KONAR-MFO on VSD Closure Under 10 kg?}

\section{Jesus Damsky Barbosa ${ }^{1}$, Liliana Ferrín ${ }^{2}$ \\ ${ }^{1}$ Pedro de Elizalde, Children's Hospital, Buenos Aires, Argentina. \\ ${ }^{2}$ Institute of Cardiology, Corrientes, Argentina}

Endovascular VSD closure is a complex intervention. In patients (p) under $10 \mathrm{~kg}$, small and flexible devices are necessary to treat these defects. The KONAR-Multifunctional Occluder (MFO) is a soft and low profile device which allows to close large diameter defects. Objective is to evaluate the feasibility and outcomes of Konar-MFO to occlude VSDs in patients less than $10 \mathrm{~kg}$ as well as the short and mid-term results. This is a prospective, descriptive and observational study. Since March 2018, endovascular VSDs closure were done in $14 \mathrm{p}$ under $10 \mathrm{~kg}$. The selection and measurements were made by transthoracic echocardiography (TTE), including left and right orifices as the VSD length taking into account Doppler color jet. The procedures were guided by transesophageal echocardiography (TEE) when it was feasible. Gender: 5 males and 9 females. All of them were under 7 months old. Weights: medium $4.6 \mathrm{~kg}$ (2900 to $10 \mathrm{~kg})$. The statistical analysis was made by t-test or student test. Statistical significance $\mathrm{p}<0.05$. Measurements: right orifice: 4 to $8 \mathrm{~mm}$; entry: 5 to $12 \mathrm{~mm}$ and length: 3 to $15 \mathrm{~mm}$. The MFO chosen was $3 \mathrm{~mm}$ bigger than the right orifice (p: 0.003). MFO implanted: 10-8: 6; 8-6: 4; 7-5: 2 and 6-4: 2 . As the association with other cardiopathies was frequent in this cohort, the approach was different depending on the association, avoiding arterial access in 9 of 14 p. In 4/14 p arteriovenous loop, $1 \mathrm{p}$ crossing the VSD without artery approach and $9 / 14 \mathrm{p}$ venous-venous loop ( 1 with inferior vena cava, 8 with right femoral and jugular veins.) $8 \mathrm{p}$ crossing an ASD, $1 \mathrm{p}$ crossing a subaortic VSD. Associations: 1p DORV with subaortic and apical VSD; 1 Subaortic VSD and apical VSD with banding of the pulmonary artery;
1 apical VSD with atrial septal defect; 1 aortic coarctation, aortic stenosis and midmuscular VSD; 1 aortic coarctation and midmuscular VSD; 1 Transposition of the Great Vessels (TGV) with midmuscular VSD; $1 \mathrm{p}$ anomalous drainage and stenosis of pulmonary veins and midmuscular VSD; 1 Gerbode postsurgical residual shunt; 1p subpulmonar VSD and 5 muscular VSDs. All the procedures were combined. 3/14 procedures were failed: 1 perimembranous VSD because of the left disc generated subaortic stenosis; 2 midmuscular VSDs due to both devices being smaller than VSD diameters; both patients had severe cardiac insufficiency. Follow-up: 2/11 p died: TGV died by sepsis; $1 \mathrm{p}$ with stenosis of pulmonary veins died during the surgery trying to correct the stenosis. The $\mathrm{p}$ with Gerbode residual shunt continues with residual shunt. All of the $p$ do not show residual shunt. (1) MFO is a good choice to close VSDs in p under $10 \mathrm{~kg}$. (2) The association with severe congenital diseases were frequent. (3) To cross directly the VSD as the venous-venous loop are excellent approaches avoiding the arterial access. (4) TTE and TEE allow to measure the VSDs and to guide the procedure. (5) Regarding muscular VSDs, the oversize was the correct decision to close them.

ETHICS DECLARATIONS

Conflict of interest: Proctor and Consultant of Lifetech Scientific. Ethical Approval: Not applicable.

Consent for Publication: Not applicable.

\section{Hybrid Versus Percutaneous Left Atrial Decompression in Infants with Hypoplastic Left Heart Variants and an Intact or Highly Restrictive Atrial Septum: A Multicenter PICES Study}

Konstantin Averin ${ }^{1,2}$, MIchael Seckeler ${ }^{3}$, Holly Bauser-Heaton ${ }^{4}$, $\overline{\text { Matthew Schwartz }}^{5}$, Paul Tannous ${ }^{6}$, Cameron Seaman ${ }^{7}$, Wendy Whiteside $^{8}$, George Nicholson', Priti Patel ${ }^{10}$, Brent Gordon ${ }^{11}$, Ryan Romans ${ }^{12}$, Rajiv Devanagondi ${ }^{13}$, Carrie Herbert ${ }^{14}$, Shawn Batlivala $^{15}$, Brian Boe ${ }^{16}$, Gurumurthy Hiremath ${ }^{17}$, Jeffrey Zampi $^{8}$

${ }^{1}$ Stollery Children's Hospital, Edmonton, Canada. ${ }^{2}$ University of Alberta, Edmonton, Canada. ${ }^{3}$ Banner University Medical Center, Tucson, USA. ${ }^{4}$ Sibley Heart Center, Atlanta, USA. ${ }^{5}$ Levine Children's Hospital, Charlotte, USA. 'Lurie Children's Hospital, Chicago, USA. ${ }^{7}$ Perth Children's Hospital, Perth, USA. ${ }^{8}$ C.S. Mott Children's Hospital, Ann Arbor, USA. ${ }^{9}$ Children's Hospital at Vanderbilt, Nashville, USA. ${ }^{10}$ Children's Hospital of Illinois, Peoria, USA. ${ }^{11}$ Loma Linda University Heart Institute, Loma Linda, USA. ${ }^{12}$ Children's Mercy, Kansas City, USA. ${ }^{13}$ University of Rochester Medical Center, Rochester, USA. ${ }^{14}$ Medical City Dallas Hospital, Dallas, USA. ${ }^{15}$ Cincinnati Children's Hospital, Cincinnati, USA. ${ }^{16}$ Nationwide Children's Hospital, Columbus, USA.

${ }^{17}$ University of Minnesota, Minneapolis, USA

Neonates with hypoplastic left heart variants with an intact or highly restrictive atrial septum (HLH-IAS) are critically ill at birth and require immediate intervention to survive. The current approach to management generally involves an emergent post-natal left atrial decompression (LAD) procedure via a percutaneous or hybrid approach, depending on center preference and provider experience. We performed a multi-center retrospective review of all neonates with HLH-IAS from January 2009 to March 2020 at 14 North American congenital cardiac programs who underwent LAD in the first $36 \mathrm{~h}$ of life. Patient and procedural characteristics, survival to hospital discharge and long-term outcomes were collected and compared between treatment strategies. Of the 132 patients with HLH-IAS, 105 $(80 \%)$ underwent percutaneous and 23 (17\%) hybrid LAD; $4(3 \%)$ 
had surgical septectomy and were excluded from further analysis. There were no significant pre-procedural differences between the groups in birth weight $(3.1 \pm 0.6 \mathrm{~kg} \vee 3.2 \pm 0.6 \mathrm{~kg}, p=0.453)$, presence of an ASD (66\% v 61\%, $p=0.659)$, ASD gradient $(14.4 \pm 6.9 \mathrm{mmHg}$ $12.7 \pm 4.6 \mathrm{mmHg}, p=0.456)$ or lowest $\mathrm{pH}$ $(7.13 \pm 0.14$ v $7.09 \pm 0.15, p=0.193)$. Hybrid LAD patients were more likely to be delivered via planned C-Sect. (96\% v $74 \%$, $p=0.031)$, had shorter time from birth to procedure $(52 \mathrm{v} 120 \mathrm{~min}$, $p=0.005$ ) and had shorter time to ASD creation (29 vs $55 \mathrm{~min}$, $p=0.002$ ). There were no differences between post-procedure oxygen levels, final ASD gradients, and final ASD size. There was a trend towards fewer complications with hybrid LAD (17\% v 36\%, $p=0.141$ ). Most patients (67\%) who underwent hybrid LAD had simultaneous pulmonary artery band placement (PAB) with an additional 3 requiring $\mathrm{PAB}$ in a subsequent procedure; $21 \%$ of percutaneous LAD patients required reoperation for PAB. All hybrid LAD were technically successful, while $10 \%$ of percutaneous LAD were not with 9 of these 11 unsuccessful procedures requiring conversion to surgical septectomy. Outcomes for percutaneous v hybrid groups were similar at a median of 20.5 vs 10 months follow upsurvival to Stage 1 palliation ( $76 \%$ vs $74 \%$ ), late survival ( 39 vs $52 \%$ ) or heart transplantation rates (11\% vs $13 \%)$. In patients with HLHIAS, a hybrid approach for LAD allows for faster and more reliable creation of an atrial septal defect with $100 \%$ technical success and less overall procedural complications compared to percutaneous LAD. The hybrid approach also allows for concomitant palliation with placement of pulmonary artery bands. Although late outcomes were similar between LAD approaches, the results highlight the overall high mortality in this high risk subset of patients with hypoplastic left heart.

ETHICS DECLARATIONS

Conflict of interest: The authors declare that they do not have any conflict of interests.

Ethical Approval: Institutional review board approval was obtained at all sites.

Consent for Publication: Not applicable.

\section{Novel Use of Drug Coated Balloons (DCB) in Stenotic Congenital or Postoperative Vascular Lesions}

\section{Adam Sinder, Sara M Trucco, Jacqueline Kreutzer}

UPMC Children's Hospital of Pittsburgh, Pittsburgh, USA

Although current interventional therapies offer high success rates for most stenotic congenital heart lesions there are a subset of patients who develop recurrent stenosis or have high risk for re-stenosis and require repeat procedures, often with limited long-term success. Numerous interventional therapies have been tried including ultrahigh pressure balloons, cutting balloons, drug eluting stents and systemic antiproliferative therapies with variable rates of success and risks. Since 2016 we used drug coated balloons (DCB) as an alternative therapy for such lesions. Explore outcomes of DCB interventions in congenital or postoperative vascular stenosis, determine risk profile and reintervention and restenosis rates. IRB approved retrospective single institution chart review study. Patient population included all patients who underwent use of DCB during catheterization procedure at UPMC Children's Hospital of Pittsburgh between January 2016 and March 2020. Demographic and procedural data were collected, including prior interventional history, vessel type (pulmonary vein, pulmonary artery, systemic vessel), original lesion, follow up cath data, percent restenosis, and safety outcomes. Measurements reviewed included pre and post intervention vessel diameters. Follow up data included repeat intervention, and results of imaging studies (echocardiogram, nuclear medicine lung perfusion study, and/or CT/MRI). 30 patients with a total combined 39 vessels were included with an average age of 8.6 years. Most common diagnoses included Tetralogy of Fallot with branch pulmonary artery stenosis, and total anomalous pulmonary venous return, although our data represented a heterogenous population. Average lumen diameter pre and post DCB intervention was 2.8 and $5.9 \mathrm{~cm}$, respectively $(p<0.0001)$. Safety profile of the 39 vessels showed no reported vascular trauma (no aneurysms, confined nor unconfined tears) attributable to the DCB. Two patients had lung reperfusion injury. Follow up ranged from 1 to 5 years. $8 / 17$ vessels that had $>2$ prior interventions did not require reintervention and did not develop symptomatic restenosis or echocardiographic evidence of restenosis with at least one year of follow up. 12/16 vessels with in-stent stenosis had no evidence of restenosis compared to $11 / 19$ vessels without stents. Additionally, $4 / 5$ vessels with congenital stenosis in the absence of surgical intervention had no evidence of recurrent stenosis compared to $12 / 25$ with prior surgical intervention on the specific vessel. Although all subgroups did not reach statistical significance $(\mathrm{P}>0.05)$, trends identified may likely show differences with a larger sample size. Furthermore, there appeared to be a trend, as displayed below, regarding the interval between catheterization procedures for individuals and vessels who had two or greater prior vessel balloon interventional procedures. Our populations showed an acceptable safety profile compared to conventional balloons with successful increased time to restenosis in a portion of the intervened vessels. No subgroup analysis was statistically significant. Future work will need to prospectively assess DCB usage in a larger sample size to determine beneficial subgroups.

\section{Pre and post drug coated balloon intervention catheterization interval for vessels with $>1$ prior catheterization}

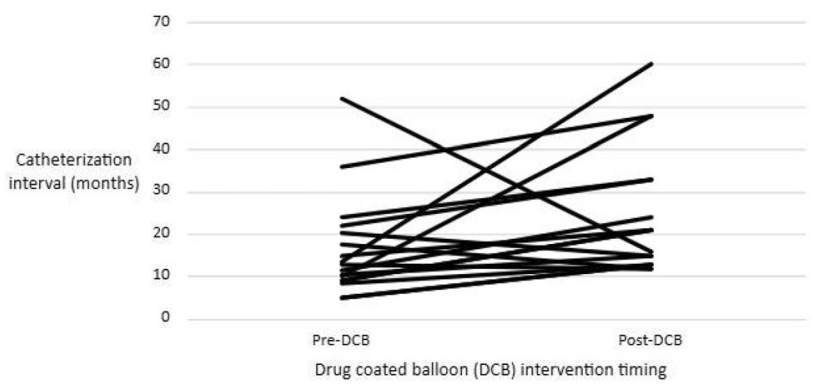

ETHICS DECLARATIONS

Conflict of interest: The authors declare that they do not have any conflict of interests.

Ethical Approval: Not applicable.

Consent for Publication: Not applicable.

\section{Coronary Ischemia and Cardiac Arrest Following Atrial Septal Defect Device Closure: An Anomalous Origin of the Coronary Artery with a Not So "Benign" Retroaortic Course}

Sergio Tolento, Luis Romero, Jose Antonio Cornejo, Jose Antonio Garcia Montes, J. Zaval, Juan Pablo Sandoval

Instituto Nacional de Cardiologia, Ciudad de Mexico, Mexico 
Anomalous origin of the coronary artery from the opposite sinus of Valsalva is a rare congenital anomaly that is characterized by an anomalous course of a native coronary vessel. The traditional definition differentiates between malignant and benign variants. The former represents an interarterial course between the pulmonary artery and aorta at risk of myocardial ischemia and lethal arrythmias, while the latter includes pre-pulmonic (in front of the pulmonary artery) and those with a retroaortic course. We report the case of an asymptomatic 4-year-old boy with a moderate to large ostium secundum atrial septal defect (ASD) with right volume overload. Relevant additional findings included dextrocardia, persistent left superior vena cava and history of pulmonary sequestration surgically repaired during infancy. The patient was scheduled to undergo elective ASD device closure. Cardiac catheterization was performed under general anesthesia. Intracardiac echo (ICE) demonstrated an $18 \mathrm{~mm}$ ASD with deficient aortic rim. Device closure was attempted initially with a $20 \mathrm{~mm}$ Amplatzer Septal Occluder (ASO). However, despite several attempts, deployment and stable position of the device was not achieved, therefore a $22 \mathrm{~mm}$ ASO was used instead. Device deployment was uneventful as seen on ICE imaging with stable position and no residual shunt thus released. Few seconds after, ST segment elevation in DI and reciprocal changes DII and DIII were noted, followed by bradycardia, complete heart block and cardiac arrest requiring immediate cardiopulmonary resuscitation. Swift snare of the right atrial disc screw allowed successful retrieval of the device restoring spontaneous blood circulation with resolution of ST-segment elevation and return to sinus rhythm. Under a stable hemodynamic condition coronary angiography and subsequent cardiac CT scan demonstrated a previously unknown anomalous origin of the coronary artery with a retroaortic course. The patient was deferred for uneventful surgical closure. Unlike a normally structured heart, an anomalous origin of the coronary artery with a retroaortic course should raise extreme awareness in patients with atrial septal defects. In the presence of a deficient anterosuperior (aortic) rim, device implantation may impinge and compress the coronary artery causing life-threatening complications.

ETHICS DECLARATIONS

Conflict of interest: The authors declare that they do not have any conflict of interests.

Ethical Approval: Not applicable.

Consent for Publication: Not applicable.

\section{Hybrid Versus Surgical Norwood Palliation for Functional Single Ventricle: Mid-to Long-Term Single Center Outcomes}

\section{Rupesh Kumar Natarajan, Sameh Said, Elizabeth Braunlin, John Bass, Gurumurthy Hiremath}

University of Minnesota Masonic Children's Hospital, Minneapolis, USA

Recent data suggested lower early survival and higher reintervention rate in higher-risk single ventricle $(\mathrm{SV})$ patients $(<34$ weeks and/ or $<2.5 \mathrm{~kg}$ at birth) after Hybrid Norwood (HN) in comparison to standard surgical approach (SN). The mid-to long-term outcomes of the hybrid approach in comparable-risk patients remains unknown. This is a retrospective single-center study comparing Hybrid stage I palliation to surgical Norwood (18 vs. 27) in neonates with SV between March 2007 and December 2020. Primary outcomes were transplant-free-survival (TFS), and re-intervention rate. The majority were beyond 34 weeks GA $(44,98 \%)$ and weighed $>2.5 \mathrm{~kg} \mathrm{(41,}$ $91 \%$ ) at birth. There was no significant difference in high-risk features between the two groups including prematurity $<34$ weeks, birth weight below $2.5 \mathrm{~kg}$, mild ventricular dysfunction, $\geq$ mild systemic $\mathrm{AV}$ valve regurgitation, hemodynamic instability or restrictive atrial septum. 31 out of 45 patients $(69 \%)$ were HLHS or its variants. Prenatal diagnosis and known associated genetic abnormality were noted in $78 \%$ and $13 \%$ respectively. The median age (GA) at stage 1 was 8 days (IQR 6, 12) with median weight of $3.3 \mathrm{~kg}(3.09,3.76)$, both comparable between $\mathrm{HN}$ and SN groups. Out of 18 patients in the hybrid arm who had a ductal stent $(6-10 \mathrm{~mm}$ in diameter), 7 needed more than one stent. Pulmonary artery (PA) bands were $3.5 \mathrm{~mm}$ in 14 patients. Classic SN was performed in 4 patients, while Norwood/Sano was done in the remaining 23. After median follow up of 823 days (IQR 312, 2359), there was no significant difference in overall TFS between Hybrid and Norwood arm (33\% vs. $48 \%$, $\mathrm{p}=0.22$ ). Overall survival was slightly lower in $\mathrm{HN}$ vs. SN (45\% vs. $70 \%, \mathrm{p}=0.08$ ) and transplantation rates were lower in HN vs. SN $(11 \%$ vs. $22 \%, p=0.34)$, but both did not meet statistical significance. Survival at 30 days $(100 \%$ vs. $96 \%, p=0.41)$, at 6 months ( 89 vs. $88 \%, p=0.96)$ and at 1 year $(72 \%$ vs. $78 \%, p=0.67)$ were all comparable between two groups. The median days to extubation (6 vs. 2 days, $\mathrm{p}=0.02$ ) and median length of hospital stay (16 vs. 34 days, $\mathrm{p}=0.008$ ) were shorter after $\mathrm{HN}$ when compared to the $\mathrm{SN}$. 35 out of 45 patients $(78 \%)$ needed a total of 70 catheter and/or surgical reinterventions. Rates of re-intervention were similar in $\mathrm{HN}$ group (15 of 18) when compared to the $\mathrm{SN}$ group (20 of 27) [83\% vs. $74 \%, \mathrm{p}=0.18]$. The most common indication for repeat surgical reoperation was recurrent arch obstruction [7/17 patients (41\%)]. Of 53 transcatheter interventions, 17 were due to PA branch stenosis $(32 \%)$ and $16(30 \%)$ were for balloon/stenting of recurrent coarctation. Overall mortality was higher in patients needing re-intervention after HN compared to after SN (53 vs. $20 \%, \mathrm{p}=0.04$ ). In this cohort of comparable risk patients, there was no significant difference in mid-to long-term outcomes between Hybrid and Norwood palliation. Although rates of re-intervention were comparable between two groups, overall mortality was significantly higher in patients needing re-intervention after a Hybrid procedure.

ETHICS DECLARATIONS

Conflict of interest: The authors declare that they do not have any conflict of interests.

Ethical Approval: Not applicable.

Consent for Publication: Not applicable.

\section{Feasibility and Safety of Arterio-Venous ECMO (SAVE study) Cannulation by Interventional Cardiologists during eCPR in Children: A Single Center, Pilot Study Results}

Ashley Kiene, MD, Cihangir Buyukgoz, MD, Hitesh Sandhu, MD, Umar Boston, MD, Christopher Knott-Craig, MD, B. Rush Waller, MD, Shyam Sathanandam, MD

University of Tennessee Health Science Center, Memphis, USA

Veno-arterial extracorporeal membrane oxygenation (VA-ECMO) cannulations have been successfully performed in adults by interventional cardiologists (IC) during cardiopulmonary resuscitation (eCPR), but little data exists in children. This study aimed to describe the safety and efficacy of ultrasound-guided percutaneous VA-ECMO cannulation performed by IC for eCPR. VA-ECMO cannulations performed by IC during eCPR between January 2018 and June 2021 in a tertiary care children's hospital were reviewed. Feasibility was defined as successful initiation of VA-ECMO following CPR without utilizing a surgical cut-down. Safety endpoints included the need for 
additional procedures related to the cannulation procedure. Fourteen VA-ECMO cannulations were attempted on thirteen patients with $100 \%$ success rate. One patient was cannulated twice at two separate CPR events. Two other patients had initiation of the cannulation procedure by the IC during CPR but was abandoned as these patients achieved hemodynamic stability before commencement of ECMO. Four other patients underwent veno-venous ECMO cannulation by the IC and were not included. Seven patients had history of congenital heart disease, one patient had idiopathic pulmonary hypertension, one patient had T-cell lymphoma and four patients had cardiomyopathy. Mean age was $10.7(0.2-18)$ years, mean weight was $48(3-109) \mathrm{kg}$. Only one patient $(3 \mathrm{~kg})$ had arterial cannulation in the carotid artery. The rest had femoral arterial cannulation. Seven cannulations $(50 \%)$ were performed with two venous drainage cannulas to support ECMO flow. A retrograde, arterial, distal perfusion cannula was also placed percutaneously in 11 of 13 femoral arterial cannulation. Median venous cannula size was $21(10-25)$ Fr and median arterial cannula size was 17 (8-17) French, and median re-perfusion cannula size was 8 (6-8) French. Median cannulation start time to ECMO flow time was 34.5 (18-112) min. Median ECMO duration was 3 (0.3-50) days. Survival at ECMO decannulation was $72 \%$ with same 30 -day survival rate as well. The overall complication rate was $21.4 \%$. One patient $(16 \mathrm{~kg})$ developed avulsion of the intima of the left common femoral artery that needed to be repaired, while 2 patients required a surgical cut down for placement of re-perfusion cannula for distal limb perfusion. It is feasible and safe to perform VA-ECMO cannulation by IC during eCPR in children. However, larger studies and further investigations are necessary especially in smaller children before advocating routine practice this strategy in children.

\section{ETHICS DECLARATIONS}

Conflict of interest: The authors declare that they do not have any conflict of interests.

Ethical Approval: Not applicable.

Consent for Publication: Not applicable.

\section{Fate of the Unligated Vertical Vein After Repair Of Infracardiac Anomalous Pulmonary Venous Connection: Transcatheter Closure of Patent Vertical Vein After Repair with a Big Device (22 mm) Amplatzer Vascular Plug-II}

\section{Carlos Guerrero, Yeny Briones, Luis Cardenas}

Hospital Calvo Mackenna, Santiago, Chile

The vertical vein (VV) may need to be left open in surgical repair of total anomalous pulmonary venous connection (TAPVC). On most occasions, the vertical vein spontaneously closes due to preferential flow to the left atrium as its compliance improves. Transcatheter closure of unligated vertical vein in supracardiac TAPVC using devices occluders has been reported. However, device closure of an unligated descending vertical vein in infracardiac TAPVC with intact atrial septum has not been previously reported. We report a case whose unligated descending vertical vein remained open following repair of infracardiac TAPVC with drainage to the portal venous system. A transcatheter closure of persistent descending vertical vein with intact atrial septum with radiofrequency (RF) transseptal perforacion technique was performed using an Amplatzer Vascular Plug II (AVP II) device (Abbott, United States) of $22 \mathrm{~mm}$. At 15-year-old $54 \mathrm{~kg}$ after follow-up with progressive fatigue appearing on moderate effort, echocardiography and CT scanner showed dilation of the right heart chambers with intact atrial septum and demonstrated a widely patent descending vertical vein of $18 \mathrm{~mm}$ of diameter. Cardiac catheterization study under general anesthesia was performed to assess Hemodynamics including quantification of the shunt by Fick's method, estimation of pulmonary vascular resistance, recording of pressures from, PA, LV, LA and trial occlusion with device. Right femoral venous and arteria access was used for the procedure. A separate femoral venous access (right) was taken to Intracardiac echocardiography (ICE) and measure the (PA) pressure. Transseptal catheterization was performed with the ICE catheter in the RA, and $\mathrm{RF}$ transseptal perforation technique. Cardiac index was $2.2 \mathrm{l} / \mathrm{min} / \mathrm{m}$ with a Qp/Qs of 1.75:1. The pulmonary vascular and systemic venous resistances were 1.33 and 24.6 indexed Wood units respectively. The mean right, left atrial (LA) and pulmonary artery (PA) pressures were normal. The left ventricle (LV) pressure was normal. Selective descending VV injections and levophase of PA showed dilated patent VV measured $18 \mathrm{~mm}$ and landing zone measure $14 \mathrm{~mm}$. Angiography at the vertical vein showed drainage is located to the portal venous system with no connection to the inferior vein cava. Subsequently, an 8 Fr Flexor Raabe Guiding Sheath (Cook Medical Corp.) by transseptal access was positioned in the VV and was occluded using a $22 \mathrm{~mm}$ AVP II. The cardiac output, PA, LA and LV pressures were measured $10 \mathrm{~min}$ after trial occlusion. The hemodynamics remained stable during trial occlusion. The $22 \mathrm{~mm}$ AVP II was deployed in the VV and its position confirmed in selective in LA injections. The patient was discharged from the hospital on the next day. Six months following the procedure, echocardiography showed no heart chamber dilatation, normal biventricular systolodiastolic function. We advised to continue oral aspirin and clopidogrel for 6 months. Late presentation of patent descending VV mimics pretricuspid shunt. Transcatheter closure through femoral approach after RF transseptal perforation technique is reasonably and effective alternative to surgical technique. The challenge appears to be not simple due to the alignment of the delivery sheath during vascular plug deployment in descending vertical vein through access transeptal. In this case was a big patent descending VV. We report a rare case of successful transcatheter closure of persistent descending VV following repair of infracardiac TAPVC using an AVP II $22 \mathrm{~mm}$. We suggest that transcatheter closure of descending VV should be considered as a treatment option (Figs. 1, 2 and 3).

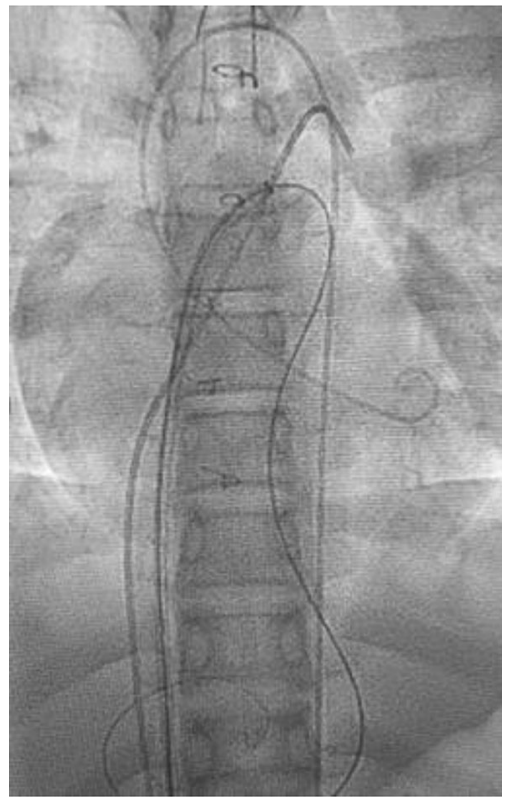

Fig. 1 Fluoroscopy steps of assess Hemodynamics 


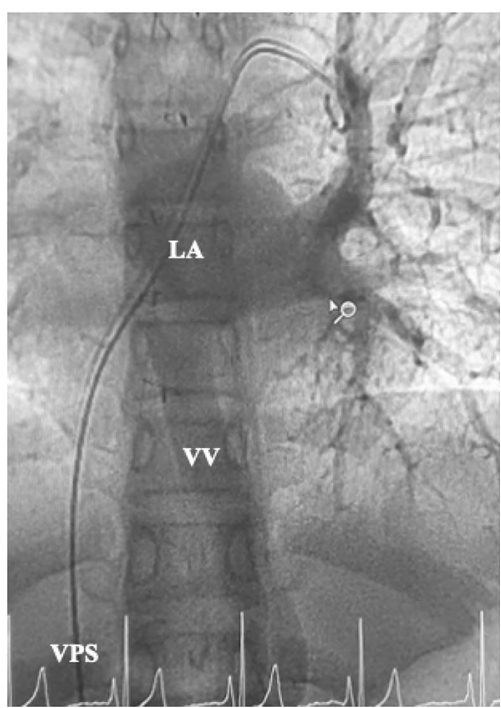

Fig. 2 PA levophase is showing pulmonary venous flow drains into (LA) and widely patent (VV). The widely patent VV drained into the portal venous system (PVS)

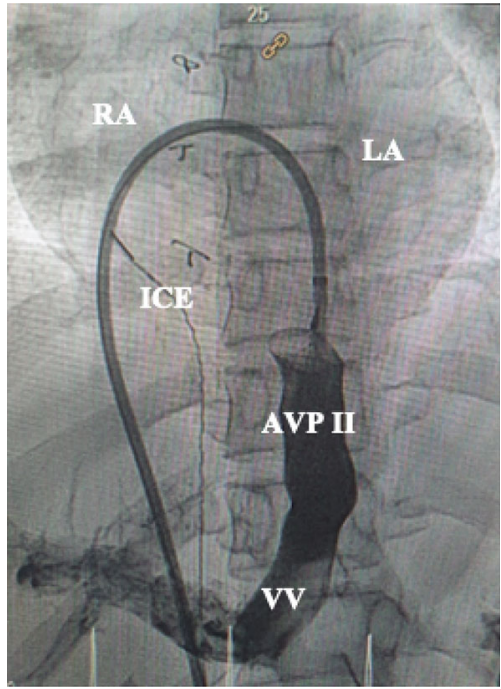

Fig. 3 Cine angiogram showing injection in the VV

\section{ETHICS DECLARATIONS}

Conflict of interest: The authors declare that they do not have any conflict of interests.

Ethical Approval: Not applicable.

Consent for Publication: Not applicable.

\section{Transhepatic Closure of Atrial Septal Defect in a child with Interrupted Inferior Vena Cava}

Celia Silva, Gustavo Feitosa Santos, Rômulo Tristão Santos, Veronica Guillen Cádima, Juliana Taguchi, Rebeca Araújo Bravo, Daniel Garoni Peternelli, Allana Lobo, Roberta François Batista, Leila Costa, Thaiza Noronha, Mariana Nunes, João Saba, Valdir Moisés, Denis Szejnfeld

UNIFESP, São Paulo, Brazil
ASD closure is routinely performed trough femoral vein access, rarely this access cannot be an option, which can occur due to anomalous drainage or blocked inferior vena cava. The transhepatic puncture is an option either through the right hepatic or the left hepatic vein. We report a case of a 9- year- old girl, with a genetic syndrome under investigation, possibly William's syndrome. Clinical examination revealed significant developmental delay, weighing only $9 \mathrm{~kg}$ and $98 \mathrm{~cm}$ of height. Her transthoracic echo showed moderated-size secundum atrial septal defect (ASD) with hemodynamic repercussion.She was sent to the Cath Lab for percutaneous closure of her ASD. During her cardiac catheterization a previously unknown systemic vein anomaly, an absent inferior vena cava was noticed. Our first option was to change the access route to the right internal jugular vein. From this access we had difficulty to manipulate the catheter across the atrial septum. The procedure was then rescheduled to try a transhepatic access. A week later she returned to the Cath Lab and under ultrasound guidance a transhepatic puncture was performed from the left lobe using a micropuncture kit NPAS-100 (Cook $\left.{ }^{\circledR}\right)$. We used a $21 \mathrm{G}$ Chiba needle $(15 \mathrm{~cm})$, with the intention to get into left hepatic vein ( LHV), which was confirmed after a short injection of contrast media. Then a 0.018 guidewire was introduced trough the needle and placed into the right atrium. A triaxial introducer was advanced over the guide wire, and then was exchanged for a 0.035 hydrophilic guide wire. The triaxial introducer was removed and a $7 \mathrm{~F}$ valved sheath was inserted. After what a $5 \mathrm{~F}$ right JR catheter was advanced, and successfully crossed into the left atrium (LA), without difficulty. Then a 0.035 extra- stiff guide wire was advanced and placed into the lefy pulmonary vein. The ASD diameter measured by a sizing -balloon (stop-flow) was $13 \mathrm{~mm}$. The sizing -balloon was removed, and a $8 \mathrm{~F}$ Mullins sheath and dilator were advanced over the guide wire. The dilator and guide wire were then removed. A $13 \mathrm{~mm}$ Amplatzer Septal Occluder device (AGA Medical corp.) was loaded and deployed successfully under transesophageal echocardiography and fluoroscopy guidance There was no residual shunt or malposition of device after its release. At the end of the procedure, we inserted the femoral dilator into the sheath and slowly pulled back the introducer to push the blood patch formed inside the introducer into the puncture course of the liver parenchyma in order to reduce the risk of bleeding. We performed a FAST ultrasound after removal of the introducer from the liver parenchyma, which showed no signs of complication. The patient was discharge home in $24 \mathrm{~h}$, without any complication. Transhepatic access for percutaneous closure of ASD is safety and a viable option. Despite the best angulation from the RHV puncture for the ASD occlusion the LHV puncture through the left hepatic lobe has the advantage of easier manual compression, and less risk of bleeding.

\section{ETHICS DECLARATIONS}

Conflict of interest: The authors declare that they do not have any conflict of interests.

Ethical Approval: Not applicable.

Consent for Publication: Not applicable.

\section{9. "One Way or Another": A Rare Case of Pulmonary Artery Supply from the Left Main Coronary Artery}

Roberto Rafael Dawson-Perry, Andrea Castillo-Gonzalez, JA Garcia-Montes, S Tolento, E Lupinta, R Querevalu, C Zabal, JP Sandoval

Instituto Nacional de Cardiologia Ignacio Chavez, Mexico City, Mexico 
Pulmonary blood flow may be supplied directly from the aorta or major aortopulmonary collaterals (MAPCAS) in approximately $20-40 \%$ of patients with pulmonary atresia with ventricular septal defect (PA-VSD). Although presence of coronary-to-pulmonary artery fistulous communications have been described, complete branch pulmonary artery supply from the coronary circulation is unusual. We present the case of a 9-month-old girl with history of cyanosis. Echo findings confirmed PA-VSD but imaging was unable to delineate accurate pulmonary blood supply. A diagnostic catheterization was performed to outline the pulmonary circulation. The angiograms revealed absent central pulmonary arteries. The origin of the left pulmonary artery (LPA) arising from an arterial duct whilst the right pulmonary artery (RPA) originated from the left main coronary artery (LMCA). In addition, two MAPCAS supplied the right mid and upper lobe segments. The latter were occluded using $6 \mathrm{~mm}$ Amplatzer Vascular Plugs (AVP) during the procedure. Two days after, the patient underwent elective repair using a $14 \mathrm{~mm}$ right ventricle-to-pulmonary artery ContegraÒ conduit (RV-PA) and VSD closure. Right pulmonary artery division at the origin of LMCA and LPA division at the ductal origin were performed and both anastomosed to the RV-PA conduit under brief cardiopulmonary bypass. During the early postoperative period, significant proximal LPA stenosis was diagnosed and successfully addressed implanting a $9 \times 16 \mathrm{~mm}$ Intrastent MegaÔLPA stent with significant decrease of the right ventricle systolic pressure to one-third systemic pressure. The patient had good recovery and postoperative course was uneventful thereafter. Pulmonary blood flow arising from the coronary circulation in patients with PA-VSD is extremely rare. Cardiac catheterization remains an undisputable diagnostic tool to properly assess pulmonary circulation in these patients and thus decide the optimal surgical strategy when undergoing complete repair.

ETHICS DECLARATIONS

Conflict of interest: The authors declare that they do not have any conflict of interests.

Ethical Approval: Not applicable.

Consent for Publication: Not applicable.

\section{Transcatheter Closure of an Unknown Fontan Fenestration Through a Coronary Sinus and Left Atrial Fistula}

\author{
Josue Diaz-Frias $^{1}$, Ahmad Khalil ${ }^{1}$, Sihong Huang ${ }^{1,2}$, Joseph \\ Vettukattil ${ }^{1,2}$ \\ ${ }^{1}$ Congenital Heart Center, Spectrum Health Helen DeVos Children's \\ Hospital, Grand Rapids, USA. ${ }^{2}$ Pediatrics and Human Development, \\ Michigan State University College of Human Medicine, Grand \\ Rapids, USA
}

The creation of a fenestration on the Fontan circulation is intended to decrease the systemic venous pressure, increase the ventricular preload, and improve the cardiac index. Nevertheless, this is at the cost of chronic hypoxemia that affects the quality of life of the individuals with this palliation. Additionally, it may present a risk factor for future thromboembolic events. In this setting, closure of the fenestration may be beneficial. Due to method variability upon surgeon predilection and institutional strategic approach, certain anatomic locations of the fenestration may become a challenge for transcatheter closure when indicated. We describe the case of a 52-year-old female with past medical history significant for tricuspid atresia type 1B, VSD and pulmonary stenosis, s/p atrio-pulmonary Fontan with sinus node dysfunction and symptomatic bradycardia that required a transvenous pacemaker implantation complicated by cardiac arrest with a thromboembolic stroke, and developmental delay. She presented with chronic cyanosis, clubbing and hepatic cirrhosis. She had an unsuccessful attempt of transcatheter fenestration closure at a different center and presented to our emergency department with diagnosis of possible malignancy. Given her significant desaturation at rest and previous stroke event, we proceeded to identify the location of the fenestration with a 3D TEE (Fig. 1). Angiographic injection into the dilated right atrium confirmed the opening of the coronary sinus (CS) into the systemic venous circuit and a large communication of the CS into the native left atrium (LA) (Fig. 2). The unrestricted flow from CS to LA was confirmed by the test occlusion of the mouth of the coronary sinus. Using a 9 French ASD delivery system, a $20 \mathrm{~mm}$ AVP 4 was advanced under fluoroscopic and echocardiographic guidance across the mouth of the coronary sinus with expansion of the distal disc and body of the occluder in the coronary sinus, and deployment of the proximal disc in the right atrium. Post-intervention there were no ischemic events to the coronary veins and the angiography showed excellent device stability and position (Fig. 3). The post-procedure course was uncomplicated with excellent echocardiographic results and the patient was discharged home the following day. Her myeloproliferation was attributed to

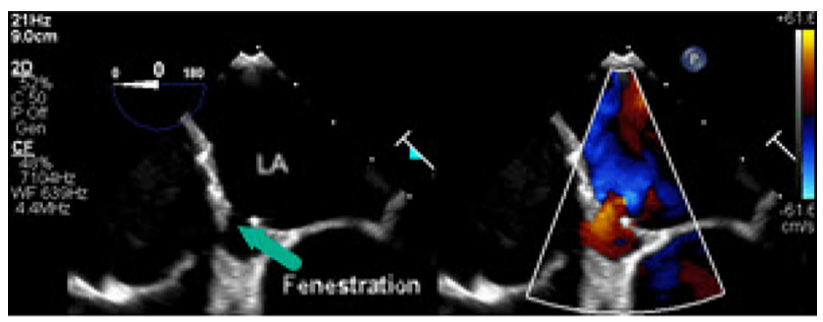

Fig. 1 .

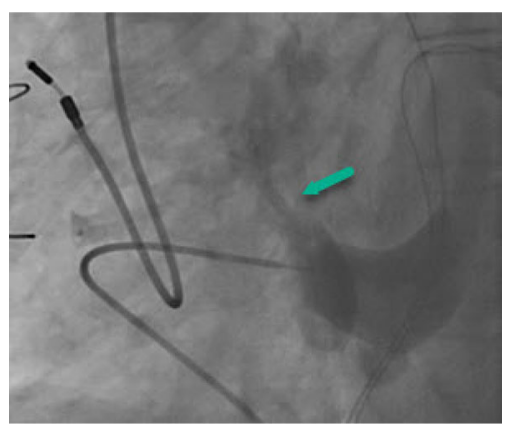

Fig. 2 .

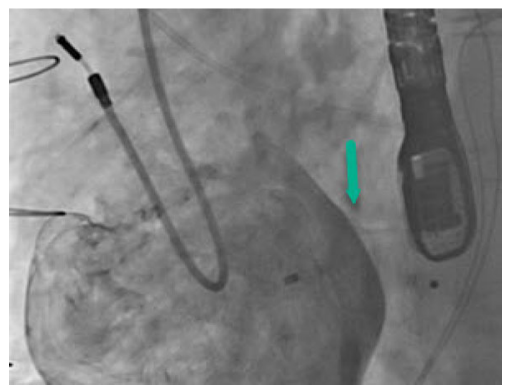

Fig. 3 . 
chronic hypoxia as the cancer evaluation was negative and she has made significant improvement after discharge.

Transcatheter closure of Fontan fenestrations with atypical locations require a detailed characterization of the shunt for an adequate device selection and determination of feasibility. The decision of performing an intervention including pacing and closing a fenestration in the Fontan palliation should be made by evaluating each case individually, assessing the benefits or disadvantages that may present in the immediate or long-term period following the procedure.

ETHICS DECLARATIONS

Conflict of interest: The authors declare that they do not have any conflict of interests.

Ethical Approval: Not applicable.

Consent for Publication: Not applicable.

\section{Transcatheter Occlusion of Left to Right Shunts in Premature Infants with Bronchopulmonary Dysplasia}

\section{Firezer Haregu, Michael McCulloch, Michael Hainstock}

University of Virginia Children's Hospital, Charlottesville, USA

Bronchopulmonary dysplasia (BPD) is a common and significant morbidity associated with prematurity, affecting $42 \%$ of infants born before 28 weeks gestation. A significant proportion of these patients require supplemental oxygen or respiratory support, as well as diuretic therapy throughout their hospitalization and sometimes at the time of discharge. It is well documented that large left to right shunting lesions (e.g. atrioventricular septal defect, truncus arteriosus, ventricular septal defects, etc.) are associated with significant pulmonary over-circulation that can worsen lung function, especially in patients with underlying lung disease. What is less clear is the impact on lung function of smaller left to right shunting lesions (specifically, atrial septal defects (ASDs) or patent ductus arteriosus (PDAs)) on premature infants with BPD. Recent trend in neonatology practice is to not surgically close ASDs or PDAs due to the risks associated with this surgery in premature infants. There have been no case-control studies to date assessing whether transcatheter occlusion of these lesions improves respiratory status in infants with BPD. To demonstrate whether transcatheter occlusion of left to right shunting lesions (i.e. ASDs and PDAs) in premature infants with BPD will result in improved respiratory status as compared to premature infants with BPD who did not have similar lesions closed. This is a retrospective case-control study in which we identified all premature infants with BPD from this institution who underwent transcatheter occlusion of ASDs or PDAs from 2015 to 2019. We then identified a control group of premature infants with BPD from this institution born between 2015 and 2019 who had a small to large ASD or PDA that never underwent surgical or transcatheter closure of the lesion. We then compared BPD severity between the two groups at monthly intervals during the first six months of life. 12 infants underwent transcatheter occlusion of either an ASD or PDA at an average age of 44 weeks corrected gestational age. All of the procedures were successful and there were no serious procedure related complications. There was no statistically significant difference in baseline demographics between the interventional group and the control group. Throughout the first six months of life, there was a greater proportion of infants who had an improvement in their BPD severity in the interventional group as compared to the control group. Our data show that transcatheter occlusion of ASDs and PDAs in premature infants is feasible and safe. It also appears BPD severity can be improved by eliminating these lesions. The data show that there is a shift towards improvement in the BPD severity in the interventional group as compared to the control group, but the number of patients in the interventional group isn't large enough to show statistical significance. We are currently adding additional patients to the interventional group to see if we can show a statistically significant difference in BPD severity between the two groups.

\section{ETHICS DECLARATIONS}

Conflict of interest: The authors declare that they do not have any conflict of interests.

Ethical Approval: Not applicable.

Consent for Publication: Not applicable.

\section{Catheter Interventions for Failed Convalescence Following Fontan Palliation}

\section{Stephen Clark, Christian Tan, Mark Law \\ University of Alabama at Birmingham, Birmingham, USA}

While early Fontan failure rates have decreased with improved patient selection, surgical techniques, and perioperative management, there continues to be significant morbidity in the early post-Fontan period. We seek to describe characteristics of patients who required unexpected early cardiac catheterization for failed typical post Fontan convalescence and the types of interventions performed. Retrospective single center chart review of all patients who underwent Fontan procedure from January 2013 to March 2021. Baseline characteristics, preoperative data, and postoperative course were reviewed. Early postoperative cardiac catheterization (EPC) was defined as unexpected catheterization occurring within three months of initial Fontan palliation. 113 patients underwent Fontan procedure during the study period. $69(61.1 \%)$ were male. Median age and weight at time of Fontan were 4.2 years (IQR 3.3, 5.3) and $16.1 \mathrm{~kg}(14.1,19.4$ ), respectively. All but one patient underwent pre-Fontan cardiac catheterization. 111/113 patients underwent placement of an extracardiac conduit, with most frequent size being $16 \mathrm{~mm}$ (77\%). Thirtyone patients $(27.4 \%)$ had Fontan fenestration. Median post-operative length of stay (LOS) was 8 days ( 7,14 days); there was one mortality and no patients listed for transplant. 26 patients $(23 \%)$ underwent EPC within 3 months of initial Fontan operation. Median time to EPC was 21 days (IQR 5, 39 days). Catheterization indications included: prolonged chest tube drainage $(18,69 \%)$, low cardiac output $(5$, $19 \%)$, unexplained hypoxia $(1,4 \%)$, plastic bronchitis $(1,4 \%)$, and acute liver injury $(1,4 \%)$. No catheter-based intervention was performed in 6 patients, with 4 patients undergoing primary surgical reintervention for Fontan takedown/revision and 2 receiving medical therapies only. 20 patients underwent 32 interventions. Common interventions included aortopulmonary collateral occlusion $(11,34 \%)$, followed by pulmonary artery balloon and/or stent angioplasty (6, $19 \%)$, SVC/innominate vein balloon and/or stent angioplasty $(5,16 \%)$ and IVC/Fontan stent angioplasty $(1,3 \%)$. Five patients underwent occlusion of residual antegrade pulmonary blood flow [MPA (3), Sano conduit (1), PDA (1)]. One patient underwent mechanical thrombolysis. Compared to patients not needing EPC, patients who underwent EPC were similar in age and weight (Table). Patients undergoing EPC had longer cardiopulmonary bypass times (median 109 vs 84 min, $p=0.014$ ) and were more likely to have had fenestration placed (50\% vs $21 \%, \mathrm{p}=0.003)$. Unsurprisingly, patients requiring catheterization had longer postoperative LOS (median 19 vs. 8 days, $\mathrm{p}<0.001)$. Patients undergoing EPC had significantly higher pulmonary vascular resistance at preoperative catheterization (1.6 vs. 1.3 Woods units, p 0.01), although other preoperative hemodynamic findings were similar. While Fontan procedure mortality is low, we demonstrate the persistence of significant 
postoperative morbidity. EPC is frequently undertaken for diagnostic and therapeutic purposes, with most common interventions being occlusion of pathways which volume- and/or pressure-load the Fontan circuit and eliminating Fontan pathway obstructions. Further study is needed to evaluate efficacy of these interventions and long-term implications.

Comparison of patients with or without early postoperative catheterization (EPC) following Fontan palliation.

\begin{tabular}{|l|c|c|c|}
\hline & $\begin{array}{c}\text { EPC } \\
\mathrm{N}=26\end{array}$ & $\begin{array}{c}\text { No EPC } \\
\mathrm{N}=87\end{array}$ & P value \\
\hline Age at Fontan, years & $4.4(3.4,5.6)$ & $4.1(3.3,5.3)$ & 0.635 \\
\hline Weight at Fontan, kg & $16.7(13.8,20.0)$ & $15.8(14.2,19.0)$ & 0.638 \\
\hline Systemic RV, yes & $16(62 \%)$ & $45(52 \%)$ & 0.378 \\
\hline Heterotaxy, yes & $5(19 \%)$ & $16(18 \%)$ & 0.923 \\
\hline Preoperative mean PAp, mm Hg* & $12(11,13.5)$ & $11(10,13)$ & 0.128 \\
\hline Preoperative PVR, Woods units* & $1.6(1.3,2.1)$ & $1.3(1.0,1.6)$ & 0.01 \\
\hline Preoperative ventricular EDP, mm Hg* & $8(8,10)$ & $9(8,10)$ & 0.30 \\
\hline Preoperative catheter intervention, yes & $12(46 \%)$ & $49(56 \%)$ & 0.461 \\
\hline CPB time, min & $109(81,142)$ & $85(63,116)$ & 0.014 \\
\hline $\begin{array}{l}\text { Additional procedure at time of Fontan, } \\
\text { yes }\end{array}$ & $12(46 \%)$ & $33(38 \%)$ & 0.452 \\
\hline Fenestration, yes & $13(50 \%)$ & $18(21 \%)$ & 0.003 \\
\hline Postoperative hospital LOS, days & $19(10,29)$ & $8(6,10)$ & $<0.001$ \\
\hline Mortality & $1(4 \%)$ & $0(0 \%)$ & 0.066 \\
\hline
\end{tabular}

Data presented as median (interquartile range).
${ }^{*} \mathrm{~N}$ does not equal full cohort

\section{ETHICS DECLARATIONS}

Conflict of interest: The authors declare that they do not have any conflict of interests.

Ethical Approval: Not applicable.

Consent for Publication: Not applicable.

\section{Comparison of U.S. Hospital Costs Between Surgical Pulmonary Valve Replacement and Transcatheter Pulmonary Valve Replacement in 2017-2019 Claims Data}

\section{Jennifer Williams, Pang Fang}

\section{Medtronic, Mounds View, USA}

Given the expansion of therapies to treat patients via transcatheter pulmonary valve replacement (TPV), there is great interest in understanding how overall inpatient hospital costs compare between TPV and surgical replacement (SPV) and how those costs continue to evolve. This analysis updates a previous one by including another year of claims data. To evaluate in-hospital costs across U.S. hospitals, we conducted a retrospective analysis of patients undergoing TPV or SPV between January 1, 2017 and December 31, 2019 using the Premier Hospital Database. Patients were included in the study if they underwent a TPV or SPV procedure based on ICD-10 procedure codes. Patients were matched 1:1 using propensity score method based on patient age, Charlson comorbidity index grouping (4 indices), gender, race, and index encounter year. In-hospital costs were defined as the total hospitalization cost including operating room, supply, room and board, ICU, lab, etc. plus pharmacy cost, adjusted to 2019 dollars. We matched 753 TPV and SPV patients across 2017, 2018, and 2019 claims years. The average hospital cost for TPV was comparable to the SPV comparator in 2017, 2018 and 2019 claims respectively (in 2019, $\mathrm{TPV}=\$ 55,944$ vs $\mathrm{SPV}=\$ 55,017$ ). The average total inpatient costs for TPV continue to be lower than SPV, despite a higher average supply cost for TPV (in 2019, TPV total $\mathrm{IP}=\$ 42,199, \mathrm{SD}=\$ 40,597$ versus $\mathrm{SPV}=\$ 53,070, \mathrm{SD}=\$ 22,979$ and TPV supply cost $=\$ 42,649, \quad \mathrm{SD}=\$ 10,091$ versus $\mathrm{SPV}=$ $\$ 16,223, \mathrm{SD}=\$ 9667)$. TPV had lower average costs in many cost centers, including room and board (in 2019, TPV $=\$ 4066$, SD $=$ $\$ 10,091$ versus $\mathrm{SPV}=\$ 17,295, \mathrm{SD}=\$ 15,400)$ and operating room (in 2019, $\mathrm{TPV}=\$ 5364, \mathrm{SD}=\$ 7349$ versus $\mathrm{SPV}=\$ 14,073, \mathrm{SD}=$ \$7299). Average length of stay for TPV continued to be significantly lower than SPV, calculating 64\% lower in 2019 claims and demonstrating $32 \%$ in an outpatient site of service. TPV continues to demonstrate comparable hospital costs to SPV, with its lower length of stay and lower costs of multiple cost centers offsetting the higher supply cost for TPV. This cost data demonstrates the necessity of hospital valve programs to consider total overall hospital costs of pulmonary valve replacement therapies versus focusing on supply costs.

\section{ETHICS DECLARATIONS}

Conflict of interest: Employee of Medtronic.

Ethical Approval: Not applicable.

Consent for Publication: Not applicable.

\section{Transcatheter Large Vessel Anastomosis for Adjacent and Non-Adjacent or Remote Vessels: Medium-Term Follow-up}

\section{Peter Guyon, Caitlin Heyden, Justin Ryan, John Moore, Howaida} EI-Said, Kanishka Ratnayaka

Division of Pediatric Cardiology, Rady Children's Hospital, UC San Diego, San Diego, USA

Transcatheter (non-surgical, closed chest) large vessel anastomosis was performed in two congenital heart disease patients. The first transcatheter anastomosis (Patient \#1) was performed in adjacent or neighboring vessels (superior vena cava and right pulmonary artery). A 35-year-old unoperated patient with functional single ventricle and limited pulmonary blood flow presented as a poor surgical candidate with New York Heart Association (NYHA) Functional Class IV heart failure symptoms. She underwent transcatheter bidirectional cavopulmonary anastomosis. The second transcatheter anastomosis (Patient \#2) was performed in non-adjacent or remote vessels (hepatic veins to azygous continuation of interrupted inferior vena cava). A 10 -year-old with prior surgical palliation of heterotaxy syndrome developed progressive cyanosis and dyspnea on exertion secondary to minimal left pulmonary artery hepatic blood flow resulting in left pulmonary arteriovenous malformations. He underwent transcatheter extra-anatomic re-routing of hepatic flow to the azygous vein [distance $=6-21 \mathrm{~mm}(\mathrm{~mm})$ varying with respiration]. We performed a retrospective review of medical records including clinic visits, echocardiograms, advanced imaging [computed tomography (CT), magnetic resonance imaging (MRI)], cardiac catheterization, and exercise stress tests through June 2021. Patient \#1 (transcatheter cavopulmonary anastomosis) has 52 months of post-procedure follow-up. She had immediate clinical improvement $(20 \mathrm{~mm}$ anastomosis; resting room-air oxygen saturation $60-70 \%$ to $85 \%$; NYHA Class IV to I) and has had no complications. Most recent echocardiogram (47 months), CT (13 months), MRI (23 months) and cardiac catheterization (38 months) demonstrated patent, unobstructed transcatheter cavopulmonary anastomosis with no stenosis or endoleak. She is being evaluated for the next stage in functional single ventricle palliation. Patient \#2 (transcatheter hepatic re-route of distant vessels) has 20 months of post-procedure follow-up. He had significant clinical improvement $(13 \mathrm{~mm}$ anastomosis; resting roomair oxygen saturation $78 \%$ to $96 \%$; NYHA Class III to I) and has had no significant complications. 3-month surveillance radiography noted stent fracture prompting elective cardiac catheterization revealing no pressure gradient, no stenosis, and no other complications. A stent (Palmaz Genesis, Cordis, Santa Clara, CA) to bridge the fractured section was deployed. Most recent echocardiogram (16 months), CT 
(9 months), and cardiac catheterization (16 months) demonstrated patent, unobstructed transcatheter cavopulmonary anastomosis with no stenosis or endoleak. Pre-intervention, he could not complete exercise stress test; post-intervention (16 months), he completed testing with minimal desaturation (4 percentage points). Mediumterm follow-up of patients undergoing transcatheter (non-surgical, closed chest) large vessel anastomosis in adjacent and non-adjacent vessels had no significant procedural, short-term, or medium-term adverse events or complications. Transcatheter anastomoses remain patent and unobstructed.

\section{ETHICS DECLARATIONS}

Conflict of interest: The authors declare that they do not have any conflict of interests.

Ethical Approval: Not applicable.

Consent for Publication: Not applicable.

\section{Comparison of Length of Stay} between Transcatheter and Surgical Pulmonary Valve Replacement claims and Harmony ${ }^{\mathrm{TM}}$ Transcatheter Pulmonary Valve (TPV) Clinical Trial

\section{Jennifer Williams, Megan Mueller, Pang Fang}

\section{Medtronic, Mounds View, USA}

In current and previous claims analyses, transcatheter pulmonary valve replacement (TPV) procedures have demonstrated lower lengths of stay than surgical pulmonary valve replacement (SPV) procedures. This analysis assesses the Harmony ${ }^{\mathrm{TM}}$ TPV Clinical Trial length of stay data to 2017-2019 claims data of TPV and SPV procedures.To evaluate in-hospital costs across U.S. hospitals, we conducted a retrospective analysis of patients undergoing TPV or SPV between January 1, 2017 and December 31, 2019 using the Premier Hospital Database. Patients were included in the study if they underwent a TPV or SPV procedure based on ICD-10 procedure codes. Patients were matched 1:1 using propensity score method based on patient age, Charlson comorbidity index grouping (4 indices), gender, race, and index encounter year. Trial data was provided via the Harmony ${ }^{\mathrm{TM}}$ studies (EFS, IDE and CAS). Claims analysis: We matched 753 TPV and SPV patients across 2017, 2018, and 2019 claims years. Average length of stay for TPV was significantly lower than SPV, calculating 64\% lower in 2019 claims and demonstrating $32 \%$ of procedures in an outpatient site of service. Mean age of patients was 29.85 and 30.29 for SPV and TPV respectively in 2019 claims. Clinical Trial analysis: 106 Harmony TPV patients were included, demonstrating a $49.3 \%$ lower length of stay than the 2019 TPV claims and $82 \%$ lower length of stay than 2019 SPV claims. Mean age of patient in the trial was 28.8. In its clinical trials, Harmony ${ }^{\mathrm{TM}} \mathrm{TPV}$ demonstrated a significantly lower length of stay than claims data for both SPV and TPV procedures. This lower length of stay may assist in offsetting the higher supply cost for TPV. This data demonstrates the necessity of hospital valve programs to consider total overall hospital costs of pulmonary valve replacement therapies, including length of stay and site of service, versus focusing on supply costs when evaluating new devices.

ETHICS DECLARATIONS

Conflict of interest: Employee of Medtronic.

Ethical Approval: Not applicable.

Consent for Publication: Not applicable.
086. Percutaneous Pulmonary Valve Implantation with Meril-Myval in Patients with Native and Large Right Ventricular Outflow Tract; Preliminary Results

Ahmet Celebi, Illker Kemal Yucel, Mustafa Orhan Bulut, İbrahim Halil Demir, Murat Surucu

Siyami Ersek Hospital for Cardiology and Cardiovascular Surgery, Department of Pediatric Cardiology, Istanbul, Turkey

We present early results of PPVI with Myval in repaired tetralogy of Fallot (TOF) patients with native-large RVOTs. $12 \mathrm{~s} / \mathrm{p}$ repaired TOF patients with native RVOT with free pulmonary regurgitation and right ventricular dilatation without significant stenosis were enrolled to study. Also, one had previous Sapien valve dysfunction. Balloon sizing was performed with compliant (34 mm Amplatzer sizing), and semicompliant balloons were used for interrogation (RVOT occlusion test). The size of the Z-Med/BIB balloons that the Andra Stents XXL would be mounted on was decided up to the indentation diameter that occurred during sizing, as at least $1 \mathrm{~mm}$ larger than the indentation diameter. If the indentation diameter was larger than $29 \mathrm{~mm}$ with sizing, RVOT occlusion test was performed with $30 \mathrm{~mm}$ semi-compliant balloons if no contrast passage during the occlusion test prestenting with $30 \mathrm{~mm}$ balloons was performed. After prestenting valve implantation was performed at the same or subsequent session. Mean age and weight of the patients were $19 \pm 7.7$ (8-32) years and $55 \pm 19(24-85) \mathrm{kg}$, respectively. Before presenting pressure gradient between the right ventricle and pulmonary artery was $5.2 \pm 3.2(0-9) \mathrm{mmHg}$. Indentation diameter with balloon sizing was $28.1 \pm 2.6(24-32.2) \mathrm{mm}$. The balloon size used for prestenting was $29.8 \pm 1.9(28-35) \mathrm{mm}$. Successful valve implantation was achieved in all patients; $32 \mathrm{~mm}$ in two, $29 \mathrm{~mm}$ in ten, and $26 \mathrm{~mm}$ (patient with valve in valve) in one. Valvulation was performed in the same session in five and 8-16 weeks after prestenting in eight. Valve function was good in all immediately after and at the last follow-up; a median of 4 months (3-7 months). Mild paravalvular leakage was observed only in one. No reintervention required yet. Myval is a bovine pericardium tri-leaflet balloon expandable valve. It is feasible and safe in patients with larger native RVOT without stenosis in adolescents and adults. Myval is currently commercially available in nine sizes; $20 \mathrm{~mm}, 21.5$, $23,24.5,26,27.5,29,30.5,32 \mathrm{~mm}$. Myval device is crimped directly on the balloon outside the body. The crimped valve with the delivery system is then loaded through 14Fr Python - Expandable Sheath. The $14 \mathrm{Fr}$ profile is used for all Myval diameters.

\section{ETHICS DECLARATIONS}

Conflict of interest: The authors declare that they do not have any conflict of interests.

Ethical Approval: Not applicable.

Consent for Publication: Not applicable.

\section{Transcatheter Interventions for Supravalvar and Branch Pulmonary Artery Stenosis After the Arterial Switch Operation}

Ahmet Celebi, İlker Kemal Yucel, Mustafa Orhan Bulut, Murat Surucu, İbrahim Halil Demir, Huseyin Karadag

Siyami Ersek Hospital for Cardiology and Cardiovascular Surgery, Department of Pediatric Cardiology, Istanbul, Turkey

Although early mortality and morbidity are low, and midterm results are excellent, many patients who undergo the arterial switch (ASO) eventually require reintervention. The pulmonary artery branches are splayed around the ascending aorta and may be compressed. Patients 
are prone to develop supravalvar (SV) and/or branch pulmonary artery (BPA) stenosis after LeCompte maneuver and they are under the risks of adverse consequences after balloon/stent procedures. We reviewed catheterization procedures performed in our institution after ASO. We performed a retrospective review of patients with d-TGA post ASO who had cardiac catheterization over a 9-year period. In 54 patients, we performed 62 procedures because of supravalvar and/or branch pulmonary artery stenosis. Indications for catheterization were determined by echocardiogram with evidence of high-pressure RV and/or narrowing of the SV or BPA.At catheterization indications for interventions included RV pressure greater than half systemic, significant angiographic narrowing, and/or pressure gradients greater than $30 \mathrm{mmHg}$. At the time of catheterization, patient mean age and weight were $5.8 \pm 4.2$ (4 months-18 years) and $23 \pm 16.9$ $(5.5-70 \mathrm{~kg}) .12$ patients were infant. Indications for catheterization were isolated BPA stenosis in 12, isolated SV in 15. Stenotic lesions were mixed (at least one BPA plus SV) in 27. 14 patients underwent cutting balloon angioplasty for BPA and among these patients, 8 has concomitant SV stenosis which underwent balloon dilation on the same session. 18 patients underwent stenting for BPA (covered in 8) whereas valve sparing SV stenting was performed in 7 (both BPA and SV stenting was performed in four). Other patients underwent balloon angioplasty with high pressure balloons (HPB) for BPA and/or SV stenosis. Acute outcomes of catheterizations in these patients included reduction of RV systolic pressure from mean of $89.1 \pm 19.6 \mathrm{mmHg}$ to $49.2 \pm 17.2 \mathrm{mmHg}$ and angiographic improvement in minimum vessel diameter. Of the 54 patients ten of them sent to surgery for ineffective balloon dilation of the SV despite very effective BPA dilation with cutting balloons and/or HBP's. Patients which are unresponsive to balloon dilation had significantly lower age and weight $(\mathrm{p}<0.05)$ when compared with responsive patients. There were four major adverse events. All were aorta-pulmonary fistula which occurred after balloon angioplasty to BPA with HBP in three and RPA stenting in one. Oversized balloon /stent dilation were used in all. Three of them sent to surgery after unsuccessful closure attempt with device/and or covered stent. Aorta to pulmonary fistula was closed with a CP covered stent in only one. No coronary compression was observed in stented patients. Other patients did not require redo surgery at a median 38 months follow up. Patients who undergo the arterial switch (ASO) may eventually require reintervention. Both angioplasty and stent implantation are effective for relieving BPA stenosis. The benefit of balloon angioplasty of the SV area can be temporary especially in infants. For SV stenosis best approach is valve sparing stent implantation. Iatrogenic AP communications after transcatheter interventions are not uncommon in patients with TGA and overdilation of the BPA's must be avoided.

ETHICS DECLARATIONS

Conflict of interest: The authors declare that they do not have any conflict of interests.

Ethical Approval: Not applicable.

Consent for Publication: Not applicable.

\section{Initial Feasibility Testing in a Swine Model of a Novel Stent and Intravascular Flow Resistor: Hope for an Entirely Percutaneous Stage 1 Palliation in Single Ventricle Congenital Heart Disease}

\section{George Nicholson, Dana Janssen, Thomas Doyle}

Vanderbilt University Medical Center, Nashville, USA

Despite great advances in surgical palliation for infants born with single ventricle heart disease, significant morbidity and mortality persist. This is particularly true for infants undergoing stage 1 palliation in the neonatal period. Our ultimate goal is to perform an entirely percutaneous stage 1 palliation utilizing devices specifically designed for this patient population. Objective is to perform initial proof of concept testing of a novel stent and intravascular flow resistor. Testing performed on a benchtop vascular simulator and then in a swine model. Benchtop testing was performed using $3 / 8^{\prime \prime}$ (outer diameter) silicone tubing with a flow rate of $11 / \mathrm{min}$ as shown in Fig. 1a. Benchtop testing was performed in both the resistor prototype and a modified micro vascular plug (Medtronic) (Fig. 1b). Initial proof of concept testing was performed in a porcine model documenting stent and intravascular resistor deployment, retrieval/ repositioning, and release. Figures 2 and 3 display the design of the novel stent and intravascular resistor prototypes. The design of these novel devices is intended to minimize apposition to the vascular wall, allow for controlled release of the device and deployment over a guidewire, and allow for device retrieval for repositioning or removal. Benchtop testing performed using an $8 \mathrm{~mm}$ intravascular resistor with a distal hole of $2.25 \mathrm{~mm}$ in the ePTFE conical membrane resulted in a pressure differential of $25 \mathrm{mmHg}$. This was comparable to a pressure differential of $28 \mathrm{mmHg}$ noted across the modified microvascular plug. In addition, the prototype device did not migrate during the
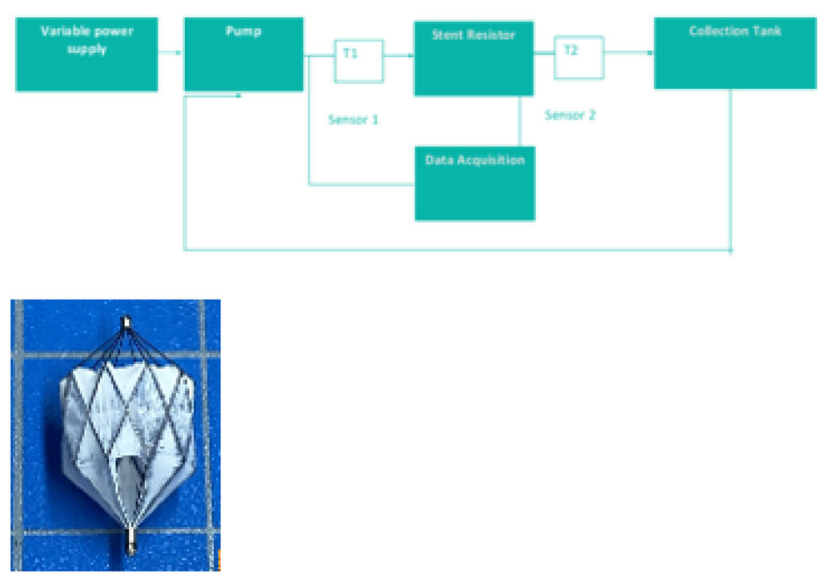

Fig. 1 (a) Benchtop test setup. (b) modified medtronic microvascular plug

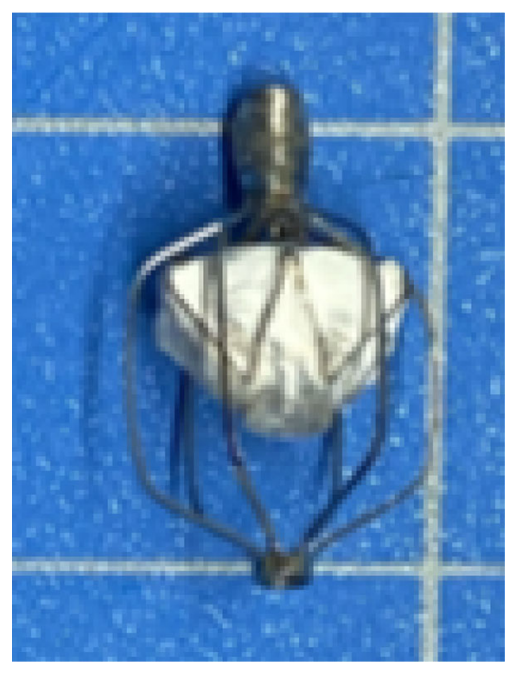

Fig. 2 Intravascular resistor design 


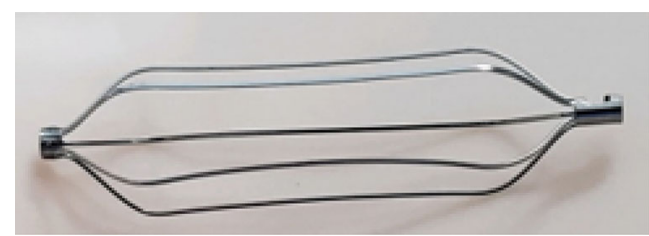

Fig. 3 Stent prototype

performance testing. In-animal testing was performed documenting feasibility of stent and intravascular resistor deployment, repositioning, and release. The measured pressure differential across the implanted resistor in a distal branch pulmonary artery was $\sim 15$ $\mathrm{mmHg}$. In addition, device retrieval after release and repositioning was able to be achieved. Further on-going in-animal testing is currently being performed to evaluate for evidence of longer-term vessel patency, development of hemolysis, and ability to remove implanted devices and evaluation for vessel wall injury. Initial proof-of-concept testing revealed feasibility of the novel design concepts in both benchtop and swine models. Further prototype refinement and inanimal testing is underway to investigate longer term viability of the implanted stent and vascular resistor devices.

ETHICS DECLARATIONS

Conflict of interest: The authors declare that they do not have any conflict of interests.

Ethical Approval: Not applicable.

Consent for Publication: Not applicable.

\section{Unique Modifications to 3D Printed Models can aid in Procedural/Surgical Planning and Patient Education in Congenital Heart Disease}

\section{Randall Bissette, Matthew Schwartz, T. Curtis Alford, Joseph Paolillo}

Sanger Heart \& Vascular Institute, Atrium Health, Charlotte, USA

$3 \mathrm{D}$ printing is a well-established technology with increasing application in medicine. Anatomically accurate congenital heart disease models have been shown to aid in surgical/procedural planning and patient education. Modifications to these models that better enable physicians to interact with them, however, are incompletely described. All patients for which a 3D printed model with a unique modification produced between 2/01/2019 and 6/01/2021 were retrospectively identified. Models were derived from patient CT scans, segmented using Materialise software, and printed on a Stratasys Objet 350 Connex 3 printer. Modifications were defined as any structure that aids in the model's presentation or usability, while preserving anatomic accuracy. These include snaps or recessed magnets for cross sectional viewing, hinges for interior viewing, and stands to present relevant anatomy in anatomically correct position for interventional planning. Relevant patient variables were extracted. 90 models were printed during the study period, 7 of which had unique modifications incorporated ( 2 blood pool models and 5 myocardial/vessel). Two blood pool models were created explicitly for patient education. First, a model of d-transposition with Mustard palliation using snaps through a modified coronal plane was created to delineate systemic vs. pulmonary venous atrium. The second teaching model was of an extracardiac Fontan circuit with complex aortic arch obstruction. The Fontan circuit and aorta were attached to each other with magnets in order to demonstrate the entire vascular anatomy while being able to disconnect the aorta for teaching purposes. Three models were created primarily to guide planning for interventional procedures.
Two myocardial/vessel models of recurrent pulmonary vein obstruction were produced, one with magnets and the other with hinges. Both demonstrated relevant areas of stenosis and aided with approaches to treatment. These were also used to educate the respective families. The other interventional model was created to assist with transcatheter aortic valve placement with unusual coronary anatomy, using an aortic valve and root model on a stand in an anatomically correct position. The last two models were printed to assist with surgical decision-making. Both models used magnets with modified coronal cuts of the myocardium to evaluate for potential bi-ventricular repair in variants of complex double outlet right ventricle. Unique physical modifications to $3 \mathrm{D}$ printed congenital heart models can improve their usability by enhancing education and assisting with procedure planning. Snaps and hinges may be printed on the models themselves, and recesses can be added to other models to accommodate glued magnets. These simple techniques can be easily employed by other 3D printing centers with minimal added materials or segmentation effort.

ETHICS DECLARATIONS

Conflict of interest: The authors declare that they do not have any conflict of interests.

Ethical Approval: Not applicable.

Consent for Publication: Not applicable.

\section{Re-intervention After the Arterial Switch Operation in Dextro-Transposition of the Great Arteries in a Single Institution}

\author{
$\underline{\text { Stephanie Acosta }}{ }^{1}$, Farimah Shariati ${ }^{1}$, Amro Alashi ${ }^{2}$, Jun Sasaki ${ }^{3}$, \\ Nao Sasaki ${ }^{3}$ \\ ${ }^{1}$ Nicklaus Children's Hospital, Miami, USA. ${ }^{2}$ Florida International \\ Univeristy, Miami, USA. ${ }^{3}$ New York Presbyterian Hospital/Weill \\ Cornell Medical Center, New York, USA
}

Dextro-transposition of the great arteries (d-TGA) is successfully palliated with the arterial switch operation (ASO). Long term follow-up is recommended due to post-operative complications associated with this operation. Previous studies have reported the incidence of re-intervention from $3.8 \%-28 \%$ with pulmonary artery (PA) balloon angioplasty and/or stenting being the most common. This study reports single center outcomes of re-intervention. Retrospective review of all patients who underwent ASO between 2003 and 2020. Demographics, pre-operative echocardiogram, pre and post-operative clinical course were reviewed. We identified 180 neonates who underwent ASO during the study period. Primary diagnoses included TGA with intact ventricular septum ( $n=107,59.4 \%)$, TGA with ventricular septal defect $(n=62,34.4 \%)$, and DORV with subpulmonary VSD $(\mathrm{n}=11,6.1 \%)$. There were 130 males $(72.2 \%), 18(10.1 \%)$ preterm births, and $8(4.5 \%)$ patients with birth weight less than $2.5 \mathrm{~kg}$. Prenatal diagnosis was established in 69 $(38.3 \%)$ patients. Median age at ASO was 5 days (mean age, $10.3 \pm 21.6$ days; range, 1 to 208 days), and median weight was $3.3 \mathrm{~kg}$ (mean weight, $3.34 \pm 0.58 \mathrm{~kg}$; range 1.7 to $7.28 \mathrm{~kg}$ ). The median postoperative length of stay was 15.5 days (range 7-181) with 2 patient deaths prior to discharge. Follow up data from all patients represented 687 cumulative patient years. There were 3 additional deaths during the follow up period. 49 (27.2\%) patients had re-intervention after the ASO with a median time of 0.49 years. There were $42 / 49$ patients underwent catheter based interventions: branch PA balloon angioplasty (15); supravalvar pulmonary stenosis (PS) balloon angioplasty (7); combined branch PA and supra-valvar PS balloon angioplasty (9); PA stent angioplasty (5); aortic arch balloon angioplasty (1); arch stent angioplasty (1); branch PA, supra-valvar PS and arch balloon angioplasty (1); branch PA and aortic arch balloon angioplasty (1); pericardiocentesis (1); left lower pulmonary 
vein angioplasty (1). There were 7/49 patients who had re-operation: RVOT reconstruction (4); right thoracic duct ligation (1); mechanical aortic valve replacement (1); removal of temporary pacing wires and drainage of mediastinitis (1). Six patients out of the 7 patients requiring reoperation first underwent diagnostic catheterization. Arterial switch operation has overall excellent survival outcome to hospital discharge. Majority of post-operative complication at this single institution was branch pulmonary artery stenosis requiring re-intervention and most of them are amendable to catheter-based intervention.

ETHICS DECLARATIONS

Conflict of interest: The authors declare that they do not have any conflict of interests.

Ethical Approval: Not applicable.

Consent for Publication: Not applicable.

\section{Surgical Closure of ASD II using an Axillary Approach: Comparison to Transcatheter Closure and Changing Trends}

\section{Robert Vincent $^{1,2}$, Markus Erb ${ }^{1}$, Joseph Giamelli ${ }^{1}$, Khanh Nguyen $^{3}$ \\ ${ }^{1}$ Boston Children's Health Physicians, Valhalla, USA. ${ }^{2}$ New York Medical College, Valhalla, USA. ${ }^{3}$ Westchester Medical Center, Valhalla, USA}

Over the last 2 decades transcatheter closure of ASD II has replaced surgical closure in many instances and has been thought preferrable when appropriate as it doesn't require cardiopulmonary bypass or a median sternotomy (the usual surgical approach). Length of stay (LOS) has also generally been significantly less with most transcatheter patients being admitted for 1 day whereas surgical LOS is commonly 3-4 days. Prior to 2018, ASD II's were preferentially closed in our institution using the transcatheter approach when feasible. Beginning 2018, surgical procedures at our center for ASD II as well as a number of other cardiopulmonary bypass procedures was commonly done utilizing an axillary incision as opposed to a median sternotomy. Patients are generally extubated in the operating room and discharged home the next day. This has led to a shift in our referrals for ASD closure to surgery. We reviewed our catheter vs. surgical comparison to assess the results given this change in practice. Between 2015and 2018, 29 patients had ASD II intervention at our institution using transcatheter closure (Transcatheter A). From 2018 to present, 42 patients have undergone ASD closure, 21 with catheter closure (Transcatheter B) and 21 with surgery using the axillary approach.

Results:

\begin{tabular}{|c|c|c|c|}
\hline & $\begin{array}{l}\text { Transcatheter } \\
\text { A }\end{array}$ & $\begin{array}{l}\text { Transcatheter } \\
\text { B }\end{array}$ & Surgical \\
\hline$\#(\mathrm{M} / \mathrm{F})$ & $29(10 / 19)$ & $21(11 / 10)$ & $21(10 / 11)$ \\
\hline $\begin{array}{l}\text { Age (years): range } \\
\text { (median) }\end{array}$ & $3-89(8.5)$ & $3-74(9)$ & $0.6-37(4)$ \\
\hline $\begin{array}{l}\text { Weight }(\mathrm{kg}) \text { : range } \\
\quad \text { (median) }\end{array}$ & $15-103$ (33) & $13-169$ (30) & $\begin{array}{l}5.8-71 \\
\quad(17.2)\end{array}$ \\
\hline $\begin{array}{l}\text { ASD size }(\mathrm{mm}) \text { : range } \\
\quad(\text { median })\end{array}$ & $6-32(13)$ & $6.5-28(11)$ & $9-35(15)$ \\
\hline $\begin{array}{l}\text { LOS days: range } \\
\text { (median) }\end{array}$ & $1-2(1)$ & $0-7$ (1) & $1-5(1)$ \\
\hline
\end{tabular}

Despite the low number of patients, we have seen a trend towards more surgical referrals vs catheter device closure especially in the last 2 years where surgical cases have surpassed catheter closure. In general, surgical patients are younger, smaller and have somewhat larger ASD's. The axillary approach with absence of an easily visible scar and absence of a metal device in the atrial septum combined with the equivalent LOS has been a driver of this change. As interventional cardiologists strive to develop procedures to replace what can be done in the operating room, we should expect that surgical approaches will also be modified to compete with the challenges we present resulting in more and better alternatives for our patients.

ETHICS DECLARATIONS

Conflict of interest: The authors declare that they do not have any conflict of interests.

Ethical Approval: Not applicable.

Consent for Publication: Not applicable.

\section{Atrial Septal Defect Device Closure in Children Under $15 \mathrm{~kg}$}

Edgar Lupinta-Paredes ${ }^{1}$, José Antonio García ${ }^{2}$, José Antonio

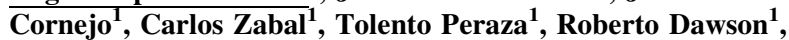
Andrea Castillo ${ }^{1}$, Randy Querevalu' ${ }^{1}$, Juan Pablo Sandoval ${ }^{1}$

${ }^{1}$ Instituto Nacional de Cardiología Ignacio Chávez, Mexico City, Mexico. ${ }^{2}$ Instituto Nacional de Cardiología Ignacio Chávez, Mexico Cy, Mexico

Transcatheter closure has been established as a widespread technique to treat most secundum atrial septal defects (ASD) in pediatric patients. There is limited data regarding the efficacy and safety of device ASD closure in smaller infants. Single-center retrospective study including all children with ASD under $\leq 15 \mathrm{~kg}$ considered for transcatheter closure at our institution between January 2009 and December 2020. Clinical, echocardiographic and interventional variables were recorded. During the study period, 834 patients underwent cardiac catheterization to attempt transcatheter closure of the ASD; $71(8 \%)$ patients weighed less than $15 \mathrm{~kg}$. Nine patients were considered non suitable candidates and no intervention was performed due to inadequate anatomy (rim deficiency) $(\mathrm{n}=3)$, pulmonary arterial hypertension $(n=3)$, and partial anomalous drainage of the pulmonary veins $(n=3)$. Sixty-two patients underwent transcatheter device closure. Mean age was $44 \pm 10$ months and median weight was 13 Kilograms (range 8-15). Median QP/QS ratio was 1.84 (range 1.58-2.46) and mean pulmonary artery pressure was $19 \pm 4 \mathrm{mmHg}$. Mean ASD diameter measured by intracardiac echocardiogram was $15 \pm 4 \mathrm{~mm}$. There has been an evolving curve in our experience closing ASD without performing stop-flow balloon sizing to determine device selection. In this cohort, we performed balloon sizing with stop-flow technique in 18 (29\%). The mean diameter of the device was $18 \pm 4 \mathrm{~mm}$ with a mean device/weight ratio of $1.4 \pm 0.3$. Successful ASD device closure occurred in 60/62 $(97 \%)$ patients. Five different devices were used to perform closure; the Amplatzer Septal Occluder $(n=51 / 60)$ was the most used occluder. In $14(22 \%)$ device repositioning and redeployment was required, and $3(5 \%)$ required a different device size to perform adequate closure. Unsuccessful closure occurred in two children, one including a deficient posterior rim despite several attempts and deploying techniques. The other patient (who was later diagnosed with an anomalous coronary artery origin with a retroaortic course) experienced coronary ischemia and cardiac arrest upon device release. Swift snare of the right atrial disc screw allowed retrieving the device restoring spontaneous circulation and sinus rhythm. No device embolizations occurred. No residual shunt was seen at 1, 6 and 
12-month follow-up echo. Transcatheter ASD device closure in children $<15 \mathrm{~kg}$ is a safe and effective.

\section{ETHICS DECLARATIONS}

Conflict of interest: The authors declare that they do not have any conflict of interests.

Ethical Approval: Not applicable.

Consent for Publication: Not applicable.

\section{Gore-Tex Valved Right Ventricular Outflow Tract Conduits Safely Expand Beyond Original Size During Percutaneous Pulmonary Valve Implantation}

\section{Alexandra Erdmann, Carlos Diaz-Castrillon, Sara Trucco, Bryan Goldstein, Victor Morell, Jacqueline Kreutzer}

UPMC Children's Hospital of Pittsburgh, Pittsburgh, USA

Percutaneous pulmonary valve implantation (PPVI) has become standard of care in the management of right ventricular outflow tract (RVOT) conduit dysfunction. Stenotic conduits often require expansion beyond the nominal size in order to accommodate for body growth and to optimize ending valve diameter. During expansion, conduit tears have been reported in up to $\sim 20 \%$ of homograft conduits (HC), which can be life-threatening. There is a paucity of data on the outcomes of PPVI in Gore-Tex valved conduits (GTC). To assess the performance of PPVI in GTC and compare with that of HC. We hypothesized that both would behave similarly regarding expandability and safety during PPVI.

Single center retrospective chart review of all patients at UPMC Children's Hospital of Pittsburgh with either a GTC or HC who underwent PPVI from 2010 to 2021. Data collection included demographics, echocardiograms, hemodynamic and angiographic data. Primary outcomes were change in conduit diameter and gradient. Secondary outcome was freedom from conduit tears. Comparative analyses were done using nonparametric tests between the two groups according to the outcome variables. Among 106 PPVIs performed at our institution, 56 were implanted into a RVOT conduit and included in the analysis $(\mathrm{GTC}=18$ and $\mathrm{HC}=39$ ). Medtronic Melody ${ }^{\mathrm{TM}}$ TPV valves were implanted in $87.5 \%(\mathrm{n}=49)$ of the patients, with the remaining being Edwards Sapien XT or S3 valves. The GTC group was younger (median 11.7 vs 23.3 years; $p<0.001$ ), smaller in weight (median $36.5 \mathrm{vs} 72.4 \mathrm{~kg} ; p=0.03$ ), and had a smaller pre-intervention conduit diameter (median 10.9 vs $13 \mathrm{~mm} ; p=0.02)$. The groups were similar regarding underlying diagnosis (majority being Tetralogy of Fallot with pulmonary atresia), indication for PPVI, and pre-procedure conduit gradients. Although both conduit types performed similarly in terms of degree of stenosis and change in gradient post-intervention (Table 1), there seemed to be a tendency towards a greater expandability capacity among the GTC group (percent change in diameter pre- to post-intervention $169.7 \%$ vs $151.7 \%, p=0.07$ and nominal to post-intervention $107.5 \%$ vs $100 \%$, $p=0.03)$. Overall, $13 \%(\mathrm{n}=7)$ of patients had conduit tears, with only 1 in the GTC group (6\% vs $16 \%$ in the HC group; $p=0.28$ ). Of the 5 conduit tears that required intervention, 4 had $\mathrm{CP}$ Numed covered stents implanted, 1 was coiled, and none required surgical repair. PPVI is safe and effective in GTC. The GTC group had a greater ability to expand beyond the nominal conduit size to accommodate PPVI with a lower incidence of conduit injury. Although the GTC patients were younger and smaller, which may have posed increased procedural challenges, the safety profile was excellent. This suggests a trend towards superiority of the GTC relative to the $\mathrm{HC}$ with regards to expandability of landing zone for PPVI beyond nominal size.

ETHICS DECLARATIONS

Conflict of interest: The authors declare that they do not have any conflict of interests.

Ethical Approval: Not applicable.

Consent for Publication: Not applicable.

\section{Feasibility, Safety, and Short-Term Outcomes of Transcatheter Patent Ductus Arteriosus Closure in Premature infants on High Frequency Jet Ventilation}

\section{Kamel Shibbani, Bassel Mohammad Nijres, Adrianne Rahde} Bischoff, Regan Giesinger, Patrick McNamara, Osamah Aldoss

\section{University of Iowa, Iowa City, USA}

Prolonged exposure to a hemodynamically significant Patent Ductus Arteriosus (PDA) is associated with major morbidity, particularly infants born less than 27 week's gestation. High Frequency Jet Ventilation (HFJV) is a standard of care at our center to minimize

Table 1 Hemodynamic and angiographic data analyses comparing Gore-Tex valved and Homograft conduits; reported as median (IQR)

\begin{tabular}{|c|c|c|c|c|}
\hline & Total & Gore-Tex & Homograft & $p$-value \\
\hline & $\mathrm{N}=56$ & $\mathrm{~N}=18$ & $\mathrm{~N}=39$ & \\
\hline \multicolumn{5}{|l|}{ Conduit diameter (mm) } \\
\hline Nominal & $20(16,23)$ & $16(16,20)$ & $22(18,24)$ & 0.005 \\
\hline Pre-intervention & $12(10,15.8)$ & $10.9(9.4,12.9)$ & $13(10.2,16.5)$ & 0.02 \\
\hline Post-intervention & $19.2(18.4,21)$ & $18.6(17.5,20)$ & $19.9(18.5,22)$ & 0.16 \\
\hline \multicolumn{5}{|l|}{ Percent change in conduit diameter $(\%)$} \\
\hline Nominal to pre-intervention & $64.1(52.6,77.5)$ & $63.2(55.6,72.7)$ & $64.1(49.6,80)$ & 0.95 \\
\hline Pre- to post-intervention & $157.4(127.9,183.5)$ & $169.7(151.2,188.7)$ & $151.7(118.6,183.5)$ & 0.07 \\
\hline Nominal to post-intervention & $100(12,110.5)$ & $107.5(100,118.75)$ & $100(16.9,109.1)$ & 0.03 \\
\hline \multicolumn{5}{|l|}{ Conduit peak gradient $(\mathrm{mmHg})$} \\
\hline Pre-intervention & $42(33,47)$ & $39(29,43)$ & $42(33,49)$ & 0.37 \\
\hline Post-intervention & $13(9,17)$ & $12(6,16)$ & $14(10,19)$ & 0.12 \\
\hline Percent Change in Conduit Gradient $(\%)$ & $32.2(23.1,44.5)$ & $30.3(26.2,38.1)$ & $33.4(22,53.9)$ & 0.48 \\
\hline
\end{tabular}


barotrauma and promote alveologenesis. There is no data related to the feasibility, safety, and outcomes of HFJV as the primary strategy during transcatheter PDA closure. This is a retrospective cohort study of premature infants with a hemodynamically significant PDA undergoing transcatheter device closure while on HFJV. The primary outcome was successful device placement. Secondary outcomes included procedure time (difference in time between sheath placement and removal), fluoroscopy time and dose, time off unit (time from departure to return to NICU), device complications, need for escalation in respiratory support, rate of pneumothorax or need for resuscitation and 7-day survival. A paired t-test was used to compare immediately-pre to 24-h post catheterization $\mathrm{FiO} 2$, mean airway pressure, and the respiratory support score (defined as mean airway pressure x FiO2). Descriptive analyses were performed on the total population. Comparison via Mann-Whitney test was performed with a small cohort of patients managed with conventional mechanical ventilation. 38 patients were included in the study. Median birth weight was $679 \mathrm{~g}$ (IQR 580-882 g). Forty seven percent of our population was born at $\leq 24$ weeks gestation. Median age at PDA catheter closure was 32 days (IQR 25.25-42.0 days) and median weight at PDA device closure was $1125 \mathrm{~g}$ (IQR 908.75-1723.50 g). There was successful device placement in $100 \%$ of patients. Venous access was successfully obtained in $100 \%$ of patients. Median procedure time was $34.50 \mathrm{~min}$ (IQR 29.25-44.25 min), median fluoroscopy time was $5.08 \mathrm{~min}$ (IQR $3.77-6.31 \mathrm{~min}$ ), and median

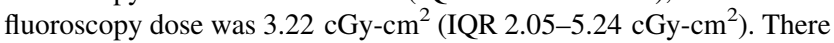
were no device complications noted and there was no pneumothorax in any of the patients. Complete PDA closure was achieved in all except one patient, who had a small residual shunt that resolved prior to discharge. Seven-day survival was $100 \%$. Only 1 patient died prior to discharge. This occurred 2 months after the procedure from an unrelated sepsis episode. There was no difference before and after catheterization in $\mathrm{FiO} 2$ requirement or respiratory support score. Mean airway pressure was significantly lower post catheterization $(\mathrm{p}<0.05)$. When comparing to a small cohort of premature infants who underwent transcatheter PDA closure with conventional mechanical ventilation, the group that underwent catheterization on a JET ventilator had shorter procedure times (33.8 min vs 42.9, $\mathrm{p}<0.05)$ and shorter "time off unit" $(103.9 \mathrm{~min}$ vs $150 \mathrm{~min}$, $\mathrm{p}<0.05)$. HFJV during transcatheter PDA closure is an effective strategy that does not compromise success rate, has excellent shortterm outcomes, and does not lead to secondary complications. Switching premature infants from HFJV to conventional ventilation might expose them to the risks of higher volumes and pressures with no tangible benefit.

\section{ETHICS DECLARATIONS}

Conflict of interest: The authors declare that they do not have any conflict of interests.

Ethical Approval: Not applicable.

Consent for Publication: Not applicable.

\section{Pulmonary Angioplasty with Oval Stent and 3D Rotational Angiography to Prevent Left Bronchium Compression in Patients with Univentricular Heart}

\section{Francisco Garay, German Bonetto, Francisco Vergara, Catherine Unzueta, Hugo Astudillo, Daniel Springmuller}

\section{UC Christus Clinical Hospital, Santiago, Chile}

Left pulmonary artery (LPA) stenosis is common after univentricular repair. It usually requires stent therapy which in turn could produce compression of the left main bronchus (LMB). An oval stent configuration has recently been proposed to avoid this complication. $3 \mathrm{D}$ rotational angiography (3DRA) has been proposed as a guide for this procedure since it demonstrates vascular anatomy and its spatial relationship with the airway. Our objective is to describe this technique and demonstrate its use in three clinical cases. Case 1: 9 yo patient with diagnosis of hypoplastic left heart syndrome requiring surgical angioplasty of pulmonary arteries during the Glenn and Fontan surgeries. MRI showed LPA stenosis and slight compression of the LMB. 3DRA confirmed anterior-posterior compression of the LPA $(8 \times 6 \mathrm{~mm})$ and bronchial compression between the LPA and the descending aorta. In addition, it demonstrated severe compression of the LMB when the LPA was dilated with a $10 \mathrm{~mm}$ balloon catheter. An Andrastent 21L stent was implanted on a Powerflex $8 \times 20 \mathrm{~mm}$ balloon, which was then reconfigured with 2 balloon catheters (Admiral Extreme $6 \times 20 \mathrm{~mm}$ and $5 \times 20 \mathrm{~mm}$ ) in a superior-inferior arrangement, obtaining the oval configuration of the stent. 3DRA evaluation showed improvement in the API $(11 \times 7 \mathrm{~mm})$, persisting distortion of the LMB without occluding it. Six months after the procedure, he remained asymptomatic and the stent is patent on echocardiogram. Case 2: 15 yo patient with diagnosis of single left ventricle, with initial Norwood procedure and Fontan surgery at 3 years of age. 3DRA confirmed anterior-posterior compression of the LPA $(11 \times 8 \mathrm{~mm})$ and bronchial compression between the LPA and the descending aorta. A Genesis 2910 stent was implanted resulting in significant compression of the LMB, so it was reconfigured with 2 balloon catheters (Powerflex $8 \times 20 \mathrm{~mm}$ and $6 \times 20 \mathrm{~mm}$ ) obtaining the oval configuration of the stent. Final 3DRA demonstrated LPA improvement $(12 \times 9 \mathrm{~mm})$ without LMB occlussion. Two years after the procedure, the stent remains patent on echocardiogram. Case 3: 16 yo patient, diagnosed with L-TGA, Ebstein-type tricuspid valve, VSD, right ventricular hipoplasia and aortic arch hypoplasia. She underwent single ventricle palliation including aortic arch augmentation with DKS anastomosis. Fontan completion was at 4 years of age which included LPA surgical angioplasty. 3DRA demonstrated anterior-posterior LPA compression $(10 \times 4.5 \mathrm{~mm})$ between a dilated aortic root and the LMB. Compression of the LMB was also demonstrated when the LPA was dilated with a $12 \times 20 \mathrm{~mm}$ Powerflex angioplasthy balloon catheter. An Andrastent 26L was then implanted on a Powerflex balloon $8 \times 40 \mathrm{~mm}$ and then it was reconfigured with 2 Powerflex balloon catheters $6 \times 20 \mathrm{~mm}$, obtaining the oval configuration of the stent. Final angiographic evaluation showed LPA improvement $(12 \times 6 \mathrm{~mm})$ without occluding the LMB. LPA angioplasty with an oval shaped stent is a new interventional resource that allows to improve LPA lumen avoiding LMB compression in patients with univentricular repair. 3DRA was able to demonstrate the compression of the LMB in the cath lab, warning about the risk of obstruction.

ETHICS DECLARATIONS

Conflict of interest: The authors declare that they do not have any conflict of interests.

Ethical Approval: Not applicable.

Consent for Publication: Not applicable.

\section{Percuteneous Closure of a "Gerbode-Like" Ventricular Septal Defect Associated with Ebstein's Anomaly}

\section{Inaki Navarro, Joaquim Miro}

Sainte-Justine Hospital, Montreal, Canada

The Ebstein's anomaly is a rare condition, and its association with a perimembranous ventricular septal defect (VSD) is even more 
uncommon. Given the close relationship of the VSD with an abnormal tricuspid valve, this association is usually sent for surgical correction. This is, to the best of our knowledge, the first report of a VSD percutaneous closure in a patient with an associated Ebstein's anomaly. We describe the case of a 3 yo patient with moderate Ebstein's anomaly and a moderate VSD that shunted into the right atrium (RA), through the atrialized portion of the right ventricle. The patient had a Ross II functional class, with mild cardiomegaly and normal arterial saturation. The echocardiogram also showed mild regurgitation of the tricuspid valve and mild to moderate dilatation of the left ventricle. A 2.5:1 Qp:Qs was measured by catheterization, demonstrating that his symptoms were mainly secondary to the VSD shunt, rather than the tricuspid valve anomaly. Under transesophageal echocardiogram (TEE) and fluoroscopy guidance, the defect was crossed and an arteriovenous loop was attempted multiple times before achieving absence of tricuspid valve interference. An $8 \mathrm{~mm}$ MFO/Konar device (Lifetech) was deployed from antegrade approach. The LV disc and occlusive lobe were deployed inside the aneurysmal sac and the RV disk in the atrialized portion of the RV. After verifying the correct position and stability of the device, and the absence of compromise of the TV, the device was released despite a moderate intra-device residual shunt. Immediate post-procedure evolution was uneventful, including absence of hemolysis. At followup echocardiograms, the residual shunt was unchanged 1 week after intervention, but subsequently reduced to mild at 1-month, to trivial at 3rd months, and disappeared at 6 months The patient improved clinically, and is now in Ross I functional class. He did not develop rhythm or conduction disorders (with a normal Holter 7 days after the VSD closure). Tricuspid regurgitation remained mild. To the best of our knowledge, this is the first case report of a percutaneous VSD closure in a patient with Ebstein's anomaly, showing its feasibility even with a tricuspid valve anomaly. Special care needs to be applied to avoid interference with the tricuspid valve, which in this case proved technically challenging. Although The MFO-Konar (Lifetech) demonstrated significant residual shunt immediately, it progressively decreased until disappearance at 6 months post-procedure. Its high conformability and softness were technically helpful for closure of the VSD in this type of anatomy, closely related to an abnormal tricuspid valve. Medical studies and residence in Mexico. Currently fellow in pediatric cardiac catheterization at Sainte-Justine Hospital in Montreal.

\section{ETHICS DECLARATIONS}

Conflict of interest: The authors declare that they do not have any conflict of interests.

Ethical Approval: Not applicable.

Consent for Publication: Not applicable.

\section{Right-Sided PAPVR Treated Percutaneously with an Amulet device: Case Report}

\author{
Inaki Navarro $^{1}$, Joaquim Miro', Mounir Riahi ${ }^{2}$ \\ ${ }^{1}$ Sainte-Justine Hospital, Montreal, Canada. ${ }^{2}$ Centre Hospitalier \\ Universitaire de Montreal, Montreal, Canada
}

Currently the most common treatment for partial anomalous pulmonary venous return (PAPVR) with a left to right shunt higher than $1.5: 1$ is surgical repair, with its intrinsic morbidity levels. However, in some cases the PAPVR has a double drainage which allows for percutaneous correction. An 11 year old patient with a VACTER association born abroad was diagnosed with a double drainage PAPVR (right lower pulmonary vein connected both to inferior vena cava and left atrium). The right cavities were enlarged and there was mild dyspnea. Cardiac SCAN showed that the communication between RLPV and IVC was short $(14 \mathrm{~mm})$ and large $(16 \mathrm{~mm})$, and close to hepatic veins. A double femoral venous access was used, to access the PAPVR from transseptal approach and IVC. Diagnostic catheterization confirmed a Qp/Qs of 2.7, due to ASD physiology (left atrium shunting to IVC, through RLPV). An initial attempt with an Occlutech PDA device (16/18) proved unsuccessful, the device being unstable and impossible to position without interference with either RLPV or IVC. Thus, an $18 \mathrm{~mm}$ Amulet Amplatzer device was placed from IVC approach. The occlusive disc of the device was deployed $4 \mathrm{~mm}$ under the RLPV, and the LA disc was in the IVC, without obstruction to it or to hepatic veins. The barbs in the occlusive disk provided sufficient stability and the device was released with no secondary displacement. At the end of the procedure a significant residual foaming through the device was observed. At follow-up, the CT-scan showed that the device fit perfectly into the defect, with no obstruction of nearby structures (RLPV, IVC and hepatic veins), while the following echocardiograms showed progressive reduction and eventual disappearance of the residual shunt. Volume of right cavities decreased and the patient normalized his exercise tolerance (Ross functional class I). This report underlines the importance of ruling out double drainage in patients with PAPVR, as this radically changes the treatment landscape. It is the first report of use of an Amulet Amplatzer device to occlude such communication. The obvious advantages of this device (shortness and enhanced stability through the occlusive disk barbs) makes it a very interesting alternative in large and complex venous malformations.

ETHICS DECLARATIONS

Conflict of interest: The authors declare that they do not have any conflict of interests.

Ethical Approval: Not applicable.

Consent for Publication: Not applicable.

\section{Stenting of a Modified Blalock-Taussig Shunt After Palliation of Complex Congenital Heart Disease: From Newborn to Infant}

\author{
Carlos Guerrero ${ }^{1}$, Daniel Perez ${ }^{2}$, Andres Fantuzzi ${ }^{2}$, Yeny \\ Briones $^{1}$, Carlos Fernandez ${ }^{2}$, Luis Cardenas ${ }^{1,2}$ \\ ${ }^{1}$ Hospital Calvo Mackenna, Santiago, Chile. ${ }^{2}$ Clinica Santa Maria, \\ Santiago, Chile
}

Contemporary surgical therapy of complex congenital heart disease (Hypoplastic Left Heart Syndrome (HLHS)- pulmonary atresia (PA) intact ventricular septum -PA with ventricular septal defect- singleventricle physiology with PA) yields excellent results. In case of early shunt Obstruction/stenosis, we previously suggested stent implantation. The purpose of this article is to report our experience with twenty patients who were treated successfully by stent implantation into the BT shunt. Hence, a surgical shunt revision could be avoided. Retrospective review including all children with stenting of a modified BT shunt in or two Centre between 2005 to 2021.10 female, age at procedure 86 days (6 days-3 years). Weight, $5.2 \mathrm{~kg}$ (2.7-18). Pulmonary Atresia with Ventricular Septal Defect 6, Hypoplastic Left Heart 5, Hypoplastic Right Heart 3, Transposition of Great Arteries with pulmonary stenosis 1 , Transposition corrected with heterotaxy and pulmonary atresia 1, others 3 . Types of coronary stents are: Multilink Vision and Prokinetic. Others stents are BeSmooth. Only 1 patient required 2 stents. 4 of them as reintervention after balloon angioplasty during follow-up. Major complications 2/16 patients with hemotorax and CPR. During the time the axillary artery approach was changed when the femoral approach was not feasible, reducing major complications. In 5 patients required another intervention in major 
catheterization such as angioplasty with stent in pulmonary arteries or occlusions of aortopulmonary collaterals. Stenting of a modified BTS is a high-risk procedure and a reasonably effective alternative to surgical technique. However, it is performed in complex patients,

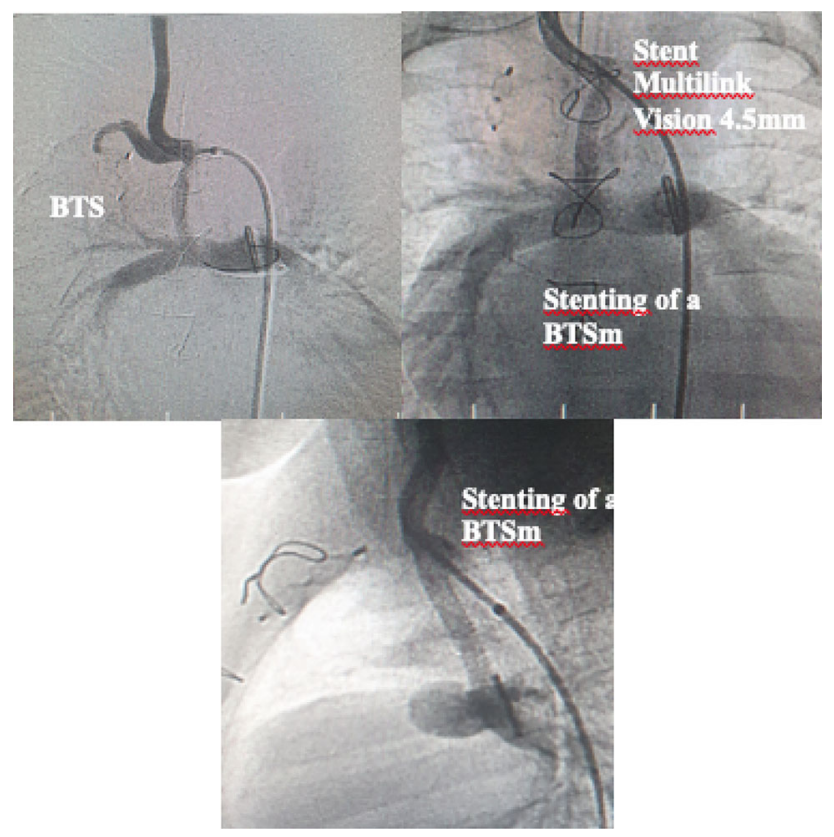

Fig. 19 months, HLHS (mitral stenosis/aortic stenosis) S/P Norwood-BTSm $3.5 \mathrm{~mm}$

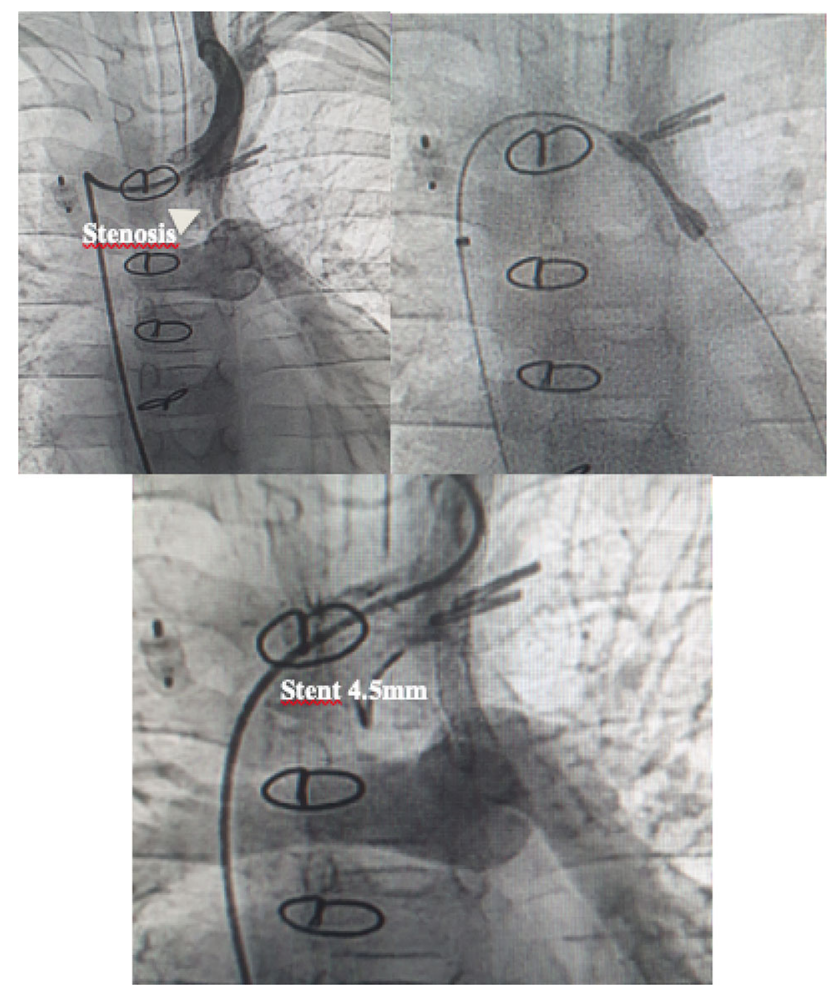

Fig. 2 Pulmonary atresia and single ventricle. BTSm $3.5 \mathrm{~mm}$ frequently univentricular, with a poor long-term prognosis, independent of the success of the intervention. Stenting of modified BT shunts in complex congenital heart disease is an option to treat shunt stenosis (Figs. 1 and 2).

\section{ETHICS DECLARATIONS}

Conflict of interest: The authors declare that they do not have any conflict of interests.

Ethical Approval: Not applicable.

Consent for Publication: Not applicable.

\section{Expansion of the Pediatric Cardiac Interventional Repertoire-Renal Artery Interventions}

\section{Angela Nguyen, Callie Brecek, Frank Ing \\ UC Davis Children's Hospital, Sacramento, USA}

While adult interventional cardiologists have expanded their procedural repertoire to include renal artery interventions, the pediatric interventional community has not followed suit. There is little data originating from pediatric cardiologists on catheterization and intervention to treat renal artery stenosis in pediatric patients. We report our experience with renal artery interventions to treat pediatric renovascular hypertension due to renal artery stenoses. A retrospective chart review was conducted on patients found to have systemic hypertension due to renal artery stenosis who underwent catheterization and intervention on the renal artery from 2/2020 to 5/2021. Data analyzed included patient profile, catheterization data, early and intermediate outcomes including pre- and post-procedure blood pressures and anti-hypertensive medications. Six patients underwent 9 interventions (6 angioplasties and 3 stent implantations) during the study period. Median age was 9.6 yrs (range $2.4-18.1 \mathrm{yrs}$ ) and median weight was $43.6 \mathrm{~kg}$ (range $15-84 \mathrm{~kg}$ ). Causes of renal artery stenoses were varied and included: fibromuscular dysplasia with renal artery stenoses and aneurysms (1), William's syndrome (1), end-stage renal disease due to vesicoureteral reflux, s/p renal transplant (1) and unknown (3), Two patients had dual arterial supply (with one vascular stenosis) to a kidney. Angioplasty (6) was performed in bilateral proximal renal arteries in 1, three regions of stenoses in a right renal artery in 1, and in a proximal left renal artery in 1 patient. Stents (3) were placed in the proximal right renal artery (2) and the proximal left renal artery (1). Median minimum diameter increased from 1.40 $(0.79-3.34) \mathrm{mm}$ to $2.56(1.24-4.86) \mathrm{mm}(\mathrm{P}<0.05)$. Drug-eluting stents $(4 \mathrm{~mm}$ diameter) were used in all. There were 2 adverse events involving renal artery spasm that resolved with local nitroglycerine administration. No other procedural complications were encountered. At clinic follow-up, $($ median $=59$ days $(48-82$ days $)$ ), average systolic blood pressure did not change significantly (from 119 to $120 \mathrm{mmHg})(\mathrm{p}=0.44)$, in spite of either eliminating or decreasing an antihypertensive medication in 4 . In 1 patient, no change in medications were made due to significant residual mid-aortic syndrome (abdominal aortic coarctation) requiring surgery. In the OR, the renal arteries were deemed to be of adequate size and did not require surgical repair. In 1 post-renal transplant patient, referral was due to CT evidence of significant renal artery stenosis at the anastomotic site. The patient was not on any antihypertensive medications prior to intervention. Follow-up catheterization at 114 days, was performed on 1 patient who had fibromuscular dysplasia with 2 aneurysms in between 3 stenotic sites. Angiography showed complete regression of the proximal aneurysm. Further angioplasty was carried out and follow-up CTA to evaluate the remaining aneurysm is pending. In this patient, antihypertensive medications were decreased from 4 to 1 post intervention. In the hands of pediatric cardiac interventionists, renal artery angioplasty and stenting is safe and effective in increasing 
vessel diameters. Antihypertensive medications can be discontinued or decreased while maintaining acceptable systolic blood pressures post intervention. Collaboration with the pediatric nephrology team is essential to develop this service. Pediatric cardiac interventionists can and should expand their procedural repertoire to include renal artery interventions. Larger series and long follow up is warranted.

\section{ETHICS DECLARATIONS}

Conflict of interest: The authors declare that they do not have any conflict of interests.

Ethical Approval: retrospective study- IRB approved.

Consent for Publication: Not applicable.

\section{Hybrid Closure of Secundum Atrial Septal Defect in Preterm Neonates and Infants: A Single Center Experience}

\section{Varun Aggarwal, Brianne Tainter, Sameh Said}

University of Minnesota Masonic Children's Hospital, Minneapolis, USA

Transcatheter atrial septal defect (ASD) closure is recommended for ostium secundum ASD but not always feasible due to patient size or vascular access issues. Hybrid ASD closure is an alternative approach in such patients and avoids cardiopulmonary bypass. We aimed to review our outcomes of hybrid ASD closure. Retrospective chart review of all hybrid closure of secundum ASD between Feb 2014 and Feb 2020 at a single center were reviewed. A total of seven pts (five females, $71 \%$ ) were identified. The indications for closure were respiratory failure secondary to chronic lung disease of prematurity and failure to thrive in all pts. All pts were born prematurely (24-29 wks gestation). One pt (14\%) had trisomy 21. Transcatheter ASD closure was not feasible due to small femoral vein size in four pts (57\%), bilateral femoral/iliac venous occlusion in one (14\%), and the need for concomitant repair of Morgagni hernia in one pt (14\%). One pt $(14 \%)$ had a prior ventriculoperitoneal shunt secondary to acute cerebellar hemorrhage. The mean age at time of intervention was $6.3 \pm 3.1$ month, while the median (IQR) weight was $8.0(4.9,12.7)$ $\mathrm{kg}$. Four pts $(57 \%)$ were on pulmonary vasodilators preoperatively. Two pts $(29 \%)$ were intubated preoperatively, while the reaming five pts $(71 \%)$ needed non-invasive ventilator support. The median ASD size was $6.5 \pm 1.3 \mathrm{~mm}$. Surgical exposure was provided via median sternotomy in two pts (29\%), subxiphoid approach in two (29\%), and right mini-thoracotomy in three pts $(43 \%)$. Amplatzer ${ }^{\text {ò }}$ septal occluder (Abbott Laboratories, IL) was the device used in all patients. The Median device size was $8 \pm 1.3 \mathrm{~mm}$. The mean procedure time was $87 \pm 69.60 \mathrm{~min}$. There was no early or late mortality. One pt (14\%) required repeat procedure two days later due to device dislodgement. Two pt (29\%) had tiny residual atrial level shunt, and two pt $(29 \%)$ required tracheostomy. The mean length of hospital stay was $6.3 \pm 5.6$ months, the mean length of hospital stay after surgery was $2.8 \pm 2.4$ months and he mean follow-up was $34.2 \pm 27.8$ months. Hybrid closure of secundum ASD is feasible in neonates and infants with low mortality and morbidity. The procedure is effective in elimination of the intracardiac shunt and facilitation of weaning from respiratory support. This approach should be considered for select patients with ostium secundum ASD but inadequate venous access.

\section{ETHICS DECLARATIONS}

Conflict of interest: The authors declare that they do not have any conflict of interests.

Ethical Approval: Not applicable.

Consent for Publication: Not applicable.

\section{Demographics and Re-Intervention Rates in Patients with Superior Vena Cava Obstruction Relieved by Transcatheter Intervention at a Single- Center}

\author{
Sarah Pradhan ${ }^{1}$, Nicholas Ollberding ${ }^{2,3}$, Nicholas Szugye ${ }^{1}$, Sarosh \\ Batlivala $^{1}$, Shabana Shahanavaz ${ }^{1}$, Russel Hirsch ${ }^{1}$ \\ ${ }^{1}$ The Heart Institute, Cincinnati Children's Hospital Medical Center, \\ Cincinnati, USA. ${ }^{2}$ Division of Biostatistics and Epidemiology, \\ Cincinnati Children's Hospital Medical Center, Cincinnati, USA. \\ ${ }^{3}$ Department of Pediatrics, University of Cincinnati College \\ of Medicine, Cincinnati, USA
}

The prevalence and etiology of superior vena cava (SVC) obstruction in congenital heart disease patients (CHD) is largely unknown, although suspected to be predominantly iatrogenic. Transcatheter based interventions have been found to relieve stenosis and have good long-term results, although there is a paucity in recent data. We retrospectively assessed the demographics of the at-risk population, immediate response to intervention and re-intervention rate.Retrospective chart review of all patients in a PedCath database and electronic health record was performed to identify SVC interventions over a 16-year period. Demographics, gradients, nature of the intervention and risk factors for re-intervention were assessed. Statistical analysis was performed using SAS Enterprise (Version 8.3) and JMP (Version 16). A total of 71 interventions were performed in 34 patients with no major complications. $58.9 \%$ of patients were male, 73.5\% had a history of CHD, and median age at initial intervention was 1 year (IQR $0.3,14.0$ ). The most common underlying etiology of SVC obstruction was post-orthotopic heart transplantation in $29.4 \%$, followed by post-extracorporeal membranous oxygenation in $20.5 \%$, post-SVC/atrial surgical baffling in $17.6 \%$ and history of chronic central venous lines in $14.7 \%$. 22 patients $(65 \%)$ underwent balloon angioplasty, and 12 underwent stenting, of whom were significantly older (median 10.5 vs 0.33 years, $p=0.0012$ ) and weighed more at the first intervention (median 28.9 vs $7.25 \mathrm{~kg}, p=0.001$ ). The mean pre- and post-intervention gradient was $7.1 \mathrm{mmHg} \pm 5.6 \mathrm{mmHg}$ vs $2.1 \mathrm{mmHg} \pm 1.9 \mathrm{mmHg}$, with no significant difference in pre-intervention gradient between groups ( $p=0.96)$, and a significantly lower post-intervention gradient after stenting ( $1.1 \mathrm{vs} 2.9 \mathrm{mmHg}, p=0.01)$. There was no significant difference in re-intervention rate between angioplasty vs stenting $(54.5 \%$ vs $41.7 \%, p=0.72)$, with $28 \%$ of angioplasty patients undergoing SVC stenting. There was no significant difference in age or weight at initial intervention or postintervention residual gradient when comparing groups at risk for future re-intervention. Etiology of SVC obstruction was not predictive of risk for re-intervention. This study identifies populations at-risk for developing SVC obstruction, with a significant proportion of patients having a history of CHD. While re-intervention is common, transcatheter intervention is safe and effectively relieves SVC obstruction, with lower gradient achieved after stenting. Age, weight, etiology of SVC obstruction and residual gradient were not risk factors for re-intervention. Careful surveillance in at-risk populations identified is crucial to ensure normal physiologic venous return. Further study is indicated to determine best strategy in the era of current stent availability.

ETHICS DECLARATIONS

Conflict of interest: The authors declare that they do not have any conflict of interests.

Ethical Approval: Not applicable.

Consent for Publication: Not applicable. 


\section{Exploring Relationships Between Echocardiographic Parameters and Cardiac Catheterization Findings in Patients with PDAs}

\author{
Omonigho Ekhomu, Saif Aljemmali, John Bokowski, Joshua \\ Murphy
}

Rush University Medical Center, Chicago, USA

Patent ductus arteriosus (PDA) is a common left-to-right shunting lesion which may be pathologic; presenting a detrimental pressure and volume load to the pulmonary circulation and volume load to the left heart. Standard of care for hemodynamically significant PDAs (HsPDAs) is transcatheter device closure; timing and decision of which is not consistently straightforward. Class I recommendations for PDA device closure include moderate or large PDAs causing enlarged left sided-chambers and heart failure. Elevated Left ventricular end diastolic dimension (LVEDD) is assumed to correlate with rising Left Ventricle End Diastolic Pressure (LVEDP); a proxy for impending heart failure. Increasing LVEDD secondary to pulmonary overcirculation as a sequelae of a significant PDA shunt may correlate with elevated LVEDP, inferring increased risk for heart failure in these patients. Determining ECHO markers that predict cardiac catheterization findings like elevated LVEDP could be helpful in deciding optimal timing for intervention in PDAs and in evaluating other cardiac lesions of interest. Ventricular- ventricular interactions are also not well understood, patients with HsPDAs may prove optimal population in which to assess. We hypothesized that increased LVEDD; a marker for LV systolic function, would predict elevated LVEDP; a marker of LV Diastolic function in patients with PDAs eligible for transcatheter closure, and that elevated LVEDP may predict elevated RVEDP (Right Ventricle End Diastolic Pressure); demonstrating ventricular-ventricular effect. We collected retrospective patient data of patients who underwent transcatheter closure of PDAs between 2007 till 202. We included patients with PDAs who underwent cardiac catheterization and with pre-catheterization ECHOs three months prior to their procedures. We excluded patients with incomplete data in regards to significant ECHO and cardiac cath data, and patients with associated complex cardiac lesions. LVEDD, LVEDD Z- score, LV ejection fraction (LV $\mathrm{EF})$ were collected from transthoracic ECHO images and reports. Cardiac catheterization findings of LVEDP, RVEDP, and Qp:Qs were extracted from PedCath Database. We evaluated all patients included as the first group, then excluded non-HsPDAs (Qp:Qs $<1.5)$ for the second group. Groups of all patients with pure PDA lesions were assessed followed by pure PDAs with Qp:Qs $>1.5$. Statistical Analysis was performed with SPSS package and statistical relationships were considered significant at $\mathrm{p}<0.05$. We assessed 185 patients. Final group included 137 patients. Ages ranged from less than one month to 88 years with a female preponderance. For all patients, there was a weak positive correlation; $\mathrm{R}=0.054$, $(\mathrm{p}=0.682)$ between LVEDD Z-score and LVEDP $(\mathrm{N}=66)$. However, there was a strong positive correlation between LVEDP and RVEDP; $R=0.771$, this was statistically significant, $(p=0.000)$. In the second group; HsPDAs the correlation was surprisingly weaker between LVEDD Z-score and LVEDP; $\mathrm{R}=0.029(\mathrm{p}=0.871)$. For pure PDAs, $\mathrm{N}=45$, we found a stronger positive correlation, $\mathrm{R}=0.146 \quad(\mathrm{p}=0.377)$. For LVEDP and RVEDP, $\mathrm{R}=0.672$ $(p=0.00)$. For pure HsPDAs, $R=0.371,(p=0.107)$ LVEDD $Z$ vs. LVEDP; the strongest positive correlation we found between our variables of primary interest. Correlation of RVEDP and LVEDP in this group was again statistically significant; $R=0.451(p=0.04)$. Our study showed a weak positive relationship between LVEDP and LVEDD Z-score which was not statistically significant but stronger when assessed in patients with HsPDAs. The weak relationship may be due to the relatively small sample size achieved in this study, and due to pediatric hearts having greater compliance with lower capacity to generate higher LVEDP compared with adults. A better powered study may show a stronger correlation that may be statistically significant. Strong correlation was found between RVEDP and LVEDP further elucidating significance of ventricular-ventricular effects. Further studies are warranted to explore these relationships and to fine-tune parameters to use noninvasive imaging to potentially predict cardiac catheterization findings in evaluation and treatment of leftsided, volume-loading cardiac lesions.

ETHICS DECLARATIONS

Conflict of interest: The authors declare that they do not have any conflict of interests.

Ethical Approval: Not applicable.

Consent for Publication: Not applicable.

\section{Transfemoral PMVSD Device Closure in Infants $\leq 10 \mathrm{~kg}$}

Dhafer Alshahrani $^{1,2}$, Niall Linnane ${ }^{1}$, Brian McCrossan ${ }^{1,3}$, Paul

${ }^{1}$ Children's Health Ireland at Crumlin, Dublin, Ireland.

${ }^{2}$ Section of Pediatric Cardiology, King Abdulaziz Medical City, Department of Cardiac Sciences, Ministry of National Guard Health Affairs, Riyadh, Saudi Arabia. ${ }^{3}$ Department of Pediatric Cardiology, Royal Belfast Hospital of Sick Children, Belfast, United Kingdom

Surgical closure of perimembranous venticular septal defect (PMVSD) is still considered the first-choice therapeutic option, especially in smaller children. Transcatheter closure of PMVSD remains challenging particularly in infants. The aim of this study is to evaluate efficacy and safety of trans-femoral PMVSD device closure in infants $\leq 10 \mathrm{~kg}$ in a single tertiary referral centre. Retrospective review of departmental databases and medical charts to define patient cohort and collect demographic, procedural and follow-up data. Between July 2014 and March 2021, 16 patients underwent attempted transfemoral PMVSD device closure (12 retrograde) at a median age of 11 months (interquartile range [IQR] 9-15.5) and a median weight of $8.3 \mathrm{~kg}$ (IQR 7.2-9.5). All patients were either symptomatic or had progressive left heart dilation. Median defect size on pre-procedural transesophageal echocardiography was $6.2 \mathrm{~mm}$ (IQR 5.7-8.3). Median device size was $6 \mathrm{~mm}$ (IQR 4.5-8). Six patients received Amplazter Duct Occluder II; 4 patients received Lifetech Symmetric Membranous VSD Occluder; 4 patients received KONAR-MF VSD Occluder; 1 patient received Lifetech Eccentric Membranous VSD Occluder and 1 patient received Occlutech PmVSD Occluder. Median procedural and fluoroscopy times were $81 \mathrm{~min}$ and $12 \mathrm{~min}$ respectively. Successful device placement was achieved in 14 patients $(87.5 \%)$. One patient developed moderate aortic valve regurgitation (AR) and tricuspid valve regurgitation (TR) upon retrograde and antegrade device deployment respectively. The device was not released and the patient was subsequently sent for elective surgical closure. The second patient developed progressive moderate AR two days post procedure, and the patient underwent surgical removal with no residual AR. One further patient developed transient hemolysis which resolved spontaneously within one week. There was no cases of device embolization and no other procedural complications, including no femoral arterial compromise. Median hospital stay was 1 day (IQR 1-1). On median follow-up of 40.5 months (IQR 25-64), none of the patients developed complete heart block. Three patients $(18.75 \%)$ had small residual shunts at latest follow-up which have not required any further intervention. Device closure of PMVSD's in children weighing $\leq 10 \mathrm{~kg}$ is feasible and safe with good procedural 
success rates. Use of both the antegrade and retrograde approaches may be necessary depending on anatomical variances.

\section{ETHICS DECLARATIONS}

Conflict of interest: The authors declare that they do not have any conflict of interests.

Ethical Approval: Not applicable.

Consent for Publication: Not applicable.

\section{Intranasal injection (I.N) X.Medication Showed Long Term Prophylactic from COVID19 Infection}

\section{Moatasem Bellah Elshareif}

\section{MOHP, Cairo, Egypt}

Following the first out break of un explained pneumonia in Wuhan, China, in late 2019, a new corona virus was identified as causative agent in January 2020, set in motion the pandemic we are witnessing all over the world. Lockdown of the country, home isolation, wearing face musk, and strictly healthy hygiene measures failed to prevent spreading of virus. In our observational cohort study on 80 healthcare worker at ALabasya Fever Hospital, Cairo, Egypt including nurse and doctor exposed daily to COVID19 infection positive patients and give care to them. We divide them in two homogeneous group A and B, both group wearing PPE (personnel protective equipment) in group B, 15 person can't wears face musk duo to difficulty breathing with mask in group A, they didn't exposed to I.N.medication but in group B, they exposed to I.N medication, taken one mle, half in each nostril with deep inspiration till they find the test in their mouth and throat. We following up both group for any clinical findings including, fever, sore throat, persistent cough, and or XRay chest findings (ground glass, pneumatic patch) ...if there's any clinical or radiological findings test for COVID19 by rtPCR was done ... if no clinical or radiological findings for more than one month after exposed to COVID19 infection positive patients, we obsolete to do rtPCR test ...we following up both group after 4, 10 days, 2 weeks, 1 month, and 2 months. The results, 17 persons from group A, were developing clinical manifestations and $\mathrm{rt}$ PCR positive...no one from group B developing any clinical manifestations even after tow month follow up ...only one who wearing face musk developing mild flu like symptoms and tested positive,he received another dose from $\mathrm{X}$ medication and give better responses ... he still give test positive for more than one month later with no any clinical manifestations ...retrospective follow up of relative to both group for mor than 3 months showing more than one relative in group A, catching the clinical manifestations and tested positive.on the other hand no one catch any clinical manifestations even in group didn't wearing face musk in group B. I.N medication X, may give good prophylactic results for person at high risk to catch COVID19 infection from infectious patients during dealing with them.and may give good prophylactic results to their relative and contacts with them..studying the role of medication in prophylactic and also management of positive COVID19 infection patients must be done on more group to be highly approved to be the solution of control spreading of virus and diseases....Keywords,. Corona virus, pandemic, infection spread, prophylactic medication.

\section{ETHICS DECLARATIONS}

Conflict of interest: The authors declare that they do not have any conflict of interests.

Ethical Approval: Not applicable.

Consent for Publication: Not applicable.
105. Incidence of Coronary Artery Compression During Attempted Percutaneous Transcatheter Pulmonary Valve Implantation After Arterial Switch Operation

Stephen Nageotte $^{1}$, Arash Salavitabar ${ }^{2}$, Jenny Zablah ${ }^{3}$, Allen Ligon $^{4}$, Mariel Turner ${ }^{5}$, Howaida El-Said ${ }^{6}$, Peter Guyon ${ }^{6}$, David Balzer $^{1}$, Shabana Shahanavaz ${ }^{7}$

${ }^{1}$ Washington University in St. Louis, Saint Louis, MO, USA. ${ }^{2}$ University of Michigan, Ann Arbor, MI, USA. ${ }^{3}$ Children's Hospital Colorado, Aurora, CO, USA. 'Joe DiMaggio Children's Hospital, Hollywood, FL, USA. ${ }^{5}$ Columbia University, New York, NY, USA. ${ }^{6}$ Rady Children's Hospital, San Diego, CA, USA. ${ }^{7}$ Cincinnati Children's Hospital, Cincinnati, OH, USA

The purpose of this study is to evaluate the efficacy, safety and effectiveness of transcatheter pulmonary valve replacement (TPVR) therapy in managing right ventricular outflow tract (RVOT) dysfunction in patients with D-Transposition of the great vessels (DTGA) after arterial switch operation (ASO). Patients with DTGA s/p repair with arterial switch can develop RVOT dysfunction with pulmonary stenosis in the annular or supra-annular position, which can lead to pressure overload on the right ventricle. In these patients, relief of the obstruction is indicated, sometimes requiring transcatheter pulmonary valve replacement (TPVR). Coronary compression is a contraindication to TPVR and occurs in 5\% of cases, more frequently in those patients with coronary abnormalities. Patients with D-TGA are more likely to have coronary abnormalities. Our goal is to determine the rate of coronary compression during attempted TPVR implantation in patients with D-TGA after ASO. This was a retrospective multi-center cohort study of patients who underwent attempted TPVR with a history of RVOT dysfunction after ASO from 2008 to 2020. Twenty patients met inclusion criteria. All but one were referred for TPVR due to significant RVOT obstruction, with 1 patient being referred for severe pulmonary regurgitation. Initial coronary anatomy was in the "usual" pattern for D-TGA in 15 patients with 5 patients having variations. Three of these had circumflex artery arising from the right coronary, 1 with a single coronary, and 1 with an anomalous origin of the left coronary from the anterior facing sinus. Coronary artery compression during balloon testing was noted in $8 / 20$ patients $(40 \%)$. Seven of these 8 patients in whom a TPVR was not implanted had "usual" coronary anatomy for D-TGA. In all of these 7 patients, there was compression noted of the left main coronary artery. There was one patient with a single coronary artery who had concern for coronary compression due to the proximity of the origin of the artery to the balloon. TPVR was successfully implanted in the 12 remaining patients, with 10 Melody valves and 2 Sapien S3 valves. In the 11 patients who had a TPVR implanted for RVOT obstruction, the RVOT gradient decreased from $46 \mathrm{mmHg}$ pre-intervention to $10 \mathrm{mmHg}$ post-intervention. Two of these patients developed endocarditis at 5 years post-implant, with one needing to be surgically removed. An additional 2 patients needed repeat TPVR, one 1 year post-implant for valve fracture and one 6 years post-implant for re-stenosis. There were no deaths. In this sample of patients with a history of D-TGA after ASO referred for TPVR, rates of coronary compression precluding valve implantation are higher than in standard patients referred for TPVR.

\section{ETHICS DECLARATIONS}

Conflict of interest: The authors declare that they do not have any conflict of interests.

Ethical Approval: was approved by the appropriate institutional review boards and certify that the study was performed in accordance with the ethical standards as laid down in the 1964 Declaration of Helsinki and its later amendments or comparable ethical standards.

Consent for Publication: Not applicable. 
106. Percutaneous Closure Of Ventricular Septal Defect Using LifetechTM Konar-MF VSD Occluder-Initial And Short-Term Multi-institutional Results

\author{
Masood Sadiq, AU Qureshi, M Yours, S Arshad, N Hyder \\ The Children's Hospital and Institute of Child Health, Lahore, \\ Pakistan
}

Transcatheter ventricular septal defect closure remains a complex procedure with potential complications like complete heart block and aortic regurgitation. The ideal device design for such intervention is still evolving. To assess the safety, efficacy and short-term outcome of ventricular septal defect closure using LifeTech ${ }^{\mathrm{TM}}$ multifunctional (KONAR-MF ${ }^{\mathrm{TM}}$ ) VSD Occluder. In a multicenter study, 44 patients with haemodynamically significant, restrictive ventricular septal defect underwent closure with the KONAR-MF ${ }^{\mathrm{TM}}$ device from April 2019 to March 2020. Clinical, echocardiographic and angiographic data were collected and reviewed. Patients were followed up at 1, 3, 6 and 12 months. The median age and weight were $8(1.7-36)$ years and $20(11-79) \mathrm{kg}$. Of 44 patients, $8(18 \%)$ had a high muscular and 36 $(82 \%)$ had a perimembranous defect, of which 6 had mild prolapse of the right coronary cusp. The median ventricular septal defect size was 8.8 (3.9-13.4) $\mathrm{mm}$. A retrograde approach was adopted in $39(88.6 \%)$ patients. Nine patients $(20.5 \%)$ had a small residual leak and there was a slight increase in aortic regurgitation in one patient. One device, which embolized to pulmonary artery was retrieved, and defect closed with a larger device. At a median follow up of 13 (5-18) months the residual leak persisted in one $(2.3 \%)$ patient. Mild aortic regurgitation in one patient remained unchanged. There were no major complications. Percutaneous closure of ventricular septal defect using KONAR-MF ${ }^{\mathrm{TM}}$ device is safe and effective in short and mid-term follow-up including selected patients with perimembranous defect and mild prolapse of the right coronary cusp.

ETHICS DECLARATIONS

Conflict of interest: The authors declare that they do not have any conflict of interests.

Ethical Approval: Not applicable.

Consent for Publication: Not applicable.

\section{Glycosylation Disorder as Cause of Recurrent Pericardial Effusion in 2-month-old Male}

\section{Liliam Aquino, Megha Patel, Stephen Girgis, Parul Jayakar, Yadira Martinez-Fernandez}

\section{Nicklaus Children's Hospital, Miami, USA}

Recurrent pericardial effusion has a broad differential diagnosis leading to challenges in workup, treatment, and counseling. Herein described is a case of a 2-month-old male who presented with a large pericardial effusion due to a rare genetic disorder. A 2-month-old male presented with febrile seizure in the setting of rhinovirus/enterovirus infection and $\mathrm{E}$. coli positive pyelonephritis. Upon presentation, he had muffled heart sounds with hemodynamic stability. CXR showed significant cardiomegaly with echocardiogram demonstrating large circumferential pericardial effusion with no ventricular collapse. He underwent initial pericardiocentesis with the removal of $83 \mathrm{ml}$ of serous fluid. Despite treatment with systemic steroids, he reaccumulated a large pericardial effusion within one week, warranting a second pericardiocentesis. Subsequently, he developed severely elevated right atrial filling pressures necessitating the third pericardiocentesis. Infectious (CMV, EBV, parvovirus, HHV6), immunologic, and genetic (karyotype, CMA, amino acids, carnitine) workup was negative. An echocardiogram demonstrated trivial pericardial effusion and he was discharged on steroids. At a 4-month follow-up, he was readmitted for failure to thrive and global developmental delay. Brain MRI showed cerebellar atrophy. Primary Immunodeficiency panel (PIP) identified two pathogenic variants in PMM2 Exon 5 (p.Phe119Leu and p.Arg141His) consistent with a diagnosis of PMM2-Congenital disorder of glycosylation (PMM2CDG). In addition, PIP was positive for COL7A1 and DOCK8 Deletion. The patient continues with small persistent pericardial effusion and is followed, outpatient. Congenital disorders of glycosylation (CDG) are a rapidly growing family of genetic diseases with PMM2-CDG being most common. PMM2 is responsible for an essential step in glycosylation, essential for bodily functions including cell recognition and protein transport. These results in multi-organ involvement with presentation ranging from early infant death to mildly affected adults. Axial hypotonia, developmental delay, and late failure to thrive are described within the first year of life. Cardiac involvement ranges from pericarditis to fatal progressive pericardial effusion. PMM2-CDG disease presents with fatal progressive pericardial effusion. Defective glycosylation of a cell surface protein on the pericardial membrane interferes with protein transport and fluid balance leading to pericardial effusion. Severe pericardial effusion has been reported in a patient who died at 7 months of life. Similarly, this patient also presented with neurologic involvement with cerebellar hypoplasia. Imaging showing cerebellar atrophy warrants further genetic and metabolic testing. Although there is no curative therapy, a multidisciplinary team is key for providing comprehensive care, and genetic counseling. The clinical manifestation of CDG-1a showed an overly broad spectrum and multi-organ involvement, cardiac manifestation like pericardial effusion and cardiomyopathy are more common in infancy. However, this presentation can be misdiagnosed for more common causes of pericarditis, like post-infectious pericarditis. We therefore highly suggest that CDG disorders should be considered in the differential diagnosis of children with an early-onset multisystem presentation, involving neurological, gastrointestinal, and cardiac systems.

ETHICS DECLARATIONS

Conflict of interest: The authors declare that they do not have any conflict of interests.

Ethical Approval: The study qualified for IRB exemption by the Western Institutional Review Board.

Consent for Publication: Not applicable.

Publisher's Note Springer Nature remains neutral with regard to jurisdictional claims in published maps and institutional affiliations. 